\title{
THE $\phi$ RECTIFIABLE SUBSETS OF THE PLANE
}

\author{
BY \\ A. P. MORSE AND JOHN F. RANDOLPH
}

Contents

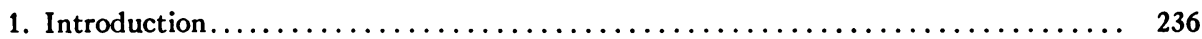

2. Definitions $\ldots \ldots \ldots \ldots \ldots \ldots \ldots \ldots \ldots \ldots \ldots \ldots \ldots \ldots \ldots \ldots \ldots \ldots \ldots \ldots \ldots \ldots \ldots \ldots \ldots \ldots, 238$

3. Measure $\ldots \ldots \ldots \ldots \ldots \ldots \ldots \ldots \ldots \ldots \ldots \ldots \ldots \ldots \ldots \ldots \ldots \ldots \ldots \ldots \ldots \ldots \ldots, 242$

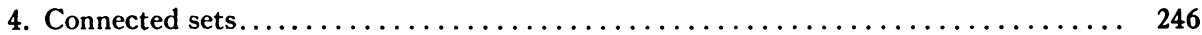

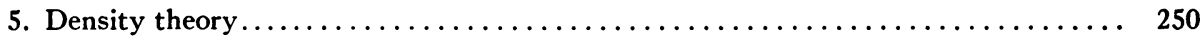

6. Rectifiability and directionality $\ldots \ldots \ldots \ldots \ldots \ldots \ldots \ldots \ldots \ldots \ldots \ldots, 262$

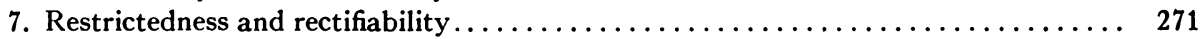

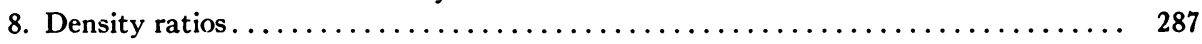

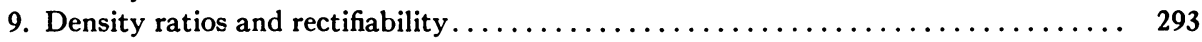

10. Rectifiability and density ratios............................. 299

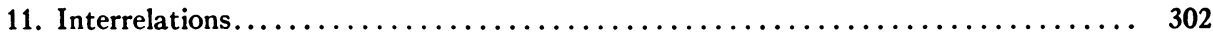

1. Introduction. Throughout the paper, as will be reaffirmed in $\$ 2$, we denote Euclidean space of two dimensions by $E_{2}$ and the family of all its subsets by $\varepsilon_{2}$.

Among the functions on $\varepsilon_{2}$ to the extended non-negative real number system there are several that for some intuitive reason or another are designated as linear measure functions (see supplementary bibliography). Of these linear measure functions, that of Carathéodory, designated by $L$ (see definitions 2.6 and 2.7), is without doubt the best known.

Besicovitch and others have made extensive studies of relations between rectifiable arcs and Carathéodory linearly measurable sets. These discussions have linear density properties as their central theme. It is natural to ask to what extent such results hold for the other linear measure functions. We answer such questions in this paper; but, rather than investigate each known linear measure function separately, we consider instead the measures of the class $\mathfrak{U}$ (see 2.3) which satisfy the first four Carathéodory axioms, and introduce various notions in terms of density relations that suggest linearity.

For $x$ a point of $E_{2}, A$ a set in $E_{2}$, and $\phi$ a measure function, the $\phi$ densities

$$
\mathfrak{D}_{\phi}^{\nabla}(A, x), \mathfrak{D}_{\phi}^{\Delta}(A, x), \mathfrak{D}_{\phi}^{\Delta}(A, x)
$$

of $A$ at $x$ are defined in 2.11. Our notions of directionality, $\phi$ directionality, restrictedness and $\phi$ restrictedness of $A$ at $x$ are also given among the definitions of $\$ 2$. Also given are the notions of rectifiability and $\phi$ rectifiability of plane sets.

Presented to the Society, October 30, 1943; received by the editors March 2, 1943, and, in revised form, September $4,1943$. 
If a set $A$ lies on a rectifiable arc and $\phi$ is a measure which in any sense is a generalization of arc length, then it is rather to be expected that

$$
0<\mathfrak{D}_{\phi}^{\nabla}(A, x)=\mathfrak{D}_{\phi}^{\Delta}(A, x)=\mathfrak{D}_{\phi}^{\Delta}(A, x)
$$

for $\phi$ almost all $x$ of $A$. On the other hand if $E_{2}$ contains $A, \phi \in \mathfrak{U}, \phi(A)<\infty$ and $\mathfrak{D}_{\phi}^{\nabla}(A, x)<\infty$ for $\phi$ almost all $x$ in $A$, then (section 11.1)

$$
\mathfrak{D}_{\phi}^{\Delta}(A, x)=\mathfrak{D}_{\phi}^{\nabla}(A, x)
$$

for $\phi$ almost all $x$ in $A$ implies that $A$ is essentially on a rectifiable arc; in fact if for $\phi$ almost all $x$ in $A$ either

$$
\mathfrak{D}_{\phi}^{\Delta}(A, x)<1.01 \mathfrak{D}_{\phi}^{\nabla}(A, x)
$$

or

$$
3 \mathfrak{D}_{\phi}^{\Delta}(A, x)<4 \mathfrak{D}_{\phi}^{\nabla}(A, x)
$$

then there is a rectifiable arc which contains all of $A$ except a subset of arbitrarily small $\phi$ measure.

A further conception of the nature of some of our results and their setting may be gained by reading 11.1, 11.2, 11.4 and 11.6.

If it is possible to inclose a non-measurable set $A$ in a measurable set $B$ with the same measure, then it is customary to elucidate the geometric properties of $A$ by a consideration of the geometric properties of $B$. However, the generality of the measures which we consider precludes this method of attack.

In order to discuss the subsets of $E_{2}$ which have finite measure, but which are otherwise arbitrary, we make considerable use of the known techniques mentioned in 3.1 and 3.3. Because of these techniques it is in general relatively unimportant to us whether or not the sets we consider are measurable. In fact we are rather inclined to more than half believe the following overstatement: Measurability information is usually a luxury, rarely a convenience, never a necessity.

We are well aware that some of our results are old; in particular, our theorems which involve descriptive restrictedness and directionality are found in a different notation on pp. 262-269 of [7]( $\left.{ }^{1}\right)$ or pp. 28-30 of [6]. However, these known results are included in the paper partly for completeness and partly to make clearer the analogy and lack of analogy between the linear descriptive and the linear metric properties of plane sets. In this connection we feel we should point out that our notation $\operatorname{dir}(A, x)$ has the same meaning which some other authors attach to the symbol $\operatorname{contg}_{A} x$.

Now it is known and we also prove in 6.12 that

$$
\mathfrak{D}_{L}^{\nabla}(A, x)=\mathfrak{D}_{L}^{\Delta}(A, x)=1
$$

(1) Numbers in brackets refer to the bibliography at the end of the paper. 
for $L$ almost all $x$ in a strictly rectifiable set $A$. From this, 5.15, and the known Theorem 3.4 we infer the known fact

$$
\mathfrak{D}_{L}^{\nabla}(A, x)=\mathfrak{D}_{L}^{\Delta}(A, x)=1
$$

for $L$ almost all $x$ in an $L$ rectifiable set $A$.

Supposing $L(A)<\infty$, we infer from 5.16 that

$$
\mathfrak{D}_{L}^{\wedge}(A, x) \leqq 1
$$

for $L$ almost all $x$; we therefore conclude from 11.1(3) and 11.1(1) that the set

$$
A \underset{x}{E}\left[3 / 4<\mathfrak{D}_{L}^{\nabla}(A, x)<1\right]
$$

has $L$ measure zero.

Accordingly we have the

ThEOREM. If $L(A)<\infty$, then the set

$$
A \underset{x}{E}\left[3 / 4<\mathfrak{D}_{L}^{\nabla}(A, x)<1\right]
$$

has $L$ measure zero.

This rather striking result of Besicovitch may be found on p. 324 of [1]. We also see at once, with the aid of 11.1(1) and 11.1(4), that the set

$$
A \underset{x}{E}\left[\mathfrak{D}_{L}^{\nabla}(A, x)=\mathfrak{D}_{L}^{\Delta}(A, x)<1\right]
$$

has $L$ measure zero whenever $L(A)<\infty$. This accords with chap. 11, pp. 324327 of Besicovitch's [1].

Thus, if in 11.1 we replace " $\phi$ " by " $L$," Theorem 11.1 becomes, in essence, a compendium of the results of Besicovitch in [1].

With three exceptions, namely, 7.5, 7.9 and 7.10, the fixed notations and definitions used in the paper are assembled in $\$ 2$. In the beginning of $\$ 2$ we list certain notations and definitions most of which are either suggestive or are in common use. Of the definitions enumerated in the remainder of $\$ 2$ the reader will require for $\$ 3$ only 2.3 through 2.8 . The paper has been written with the idea in mind that the reader will make no more than a cursory examination of 2.1 through 2.27 before beginning his perusal of $\$ 3$.

2. Definitions. Throughout the paper we use $E_{1}$ and $E_{2}$ for Euclidean space of one and two dimensions respectively, and $\varepsilon_{2}$ for the family of all subsets of $E_{2}$. For $x$ in $E_{2}$ we define the real numbers $x_{1}, x_{2}$ and $|x|$ so that $x=\left(x_{1}, x_{2}\right)$ and $|x|=\left(x_{1}^{2}+x_{2}^{2}\right)^{1 / 2}$. We shall regard $E_{2}$ and the finite complex plane as the same with $i=(0,1)$ and $i^{2}=(-1,0)$.

We regard the empty set and the number 0 as the same.

The notational phrase $x \in A$ is read $x$ is a member of $A ; x \notin A$ is read $x$ 
is not a member of $A$; and $A \subset B$ is read $A$ is a subset of $B$. For $A \subset E_{2}$ the set $E_{2}-A$ is denoted by $\tilde{A}$.

The set $E_{1} \cdot E_{t}[a \leqq t \leqq b]$ is denoted by $[a, b]$. We say $J$ is a closed interval if and only if $J$ is of the form $[a, b]$ where $-\infty<a<b<\infty$. We say $J$ is an open interval if and only if $J$ is of the form $E_{t}[a<t<b]$ where $-\infty<a<b<\infty$.

We use $\{x\}$ as an abbreviation for $E_{y}[y=x]$ and use \{\} only in this sense. Note that $\{x\}$ is the set whose sole element is $x$.

Whenever $A$ is a nonvacuous subset of $E_{2}$ we define diam $(A)$ as the supremum of numbers of the form $|x-y|$ where $x \in A$ and $y \in A$; if $A=0$ we define diam $(A)=0$. Also we use clsr $(A)$ for the closure of $A$ and bndr $(A)$ for the boundary of $A$. Thus $\operatorname{bndr}(A)=(\operatorname{clsr} A)(\operatorname{clsr} \tilde{A})$.

For $A \subset E_{2}$ and $B C E_{2}$ we define dist $(A, B)$ as the infimum of numbers of the form $|x-y|$ where $x \in A$ and $y \in B$.

We say $A$ is disconnected if and only if $A \subset E_{2}$ and there exist non-empty subsets $C_{1}$ and $C_{2}$ of $A$ such that

$$
A=C_{1}+C_{2} \text { and } C_{1}\left(\operatorname{clsr} C_{2}\right)+C_{2}\left(\operatorname{clsr} C_{1}\right)=0 .
$$

A set $A$ is said to be connected if and only if $A \subset E_{2}$ and $A$ is not disconnected. A closed connected set is said to be a continuum.

If $a_{x}^{\prime}$ is a set for $x$ in a set $B$, then

$$
\sum_{x \in B} a_{x} \text { and } \prod_{x \in B} a_{x}
$$

are so defined that

$$
\begin{aligned}
& \sum_{x \in B} a_{x}=\underset{y}{E}\left[y \in a_{x} \text { for some } x \in B\right], \\
& \prod_{x \in B} a_{x}=\underset{y}{E}\left[y \in a_{x} \text { for each } x \in B\right] .
\end{aligned}
$$

In connection with set theoretic sums we point out that

$$
\mathfrak{F}=\sum_{\bullet \in \mathfrak{F}}\{s\}
$$

whereas

$$
\underset{x}{E}[x \in s \text { for some } s \in \mathfrak{F}]=\sum_{s \in \mathfrak{F}} s .
$$

In fact we here and now define the more convenient notation $\sigma(\mathfrak{F})$ by

$$
\sigma(\mathfrak{F})=\sum_{\mathfrak{B} \in \mathfrak{F}} s .
$$

It should be noted that $\sigma(\{y\})=y$. Likewise

$$
\sigma\left(\sum_{x \in B} a_{x}\right)=\sum_{x \in B} \sigma\left(a_{x}\right)
$$


If $a_{x} \geqq 0$ for $x$ in a countable set $B$ then

$$
\sum_{x \in B} a_{x}
$$

will also be used to denote the appropriate numerical sum. Whether numerical summation or point set summation is intended will be obvious from the context.

A family $\mathfrak{F}$ is said to cover $A$ if and only if $A \subset \sigma(\mathfrak{F})$.

In this paper we call a set $A$ a Borel set if and only if it is a member of every subfamily $\mathfrak{F}$ of $\varepsilon_{2}$ for which:

(i) $B \in \mathfrak{F}$ whenever $B$ is a closed subset of $E_{2}$;

(ii) $\sigma(\mathbb{S}) \in \mathfrak{F}$ whenever $\mathbb{B}$ is a countable subfamily of $\mathfrak{F}$;

(iii) $\widetilde{B} \in \mathfrak{F}$ whenever $B \in \mathfrak{F}$.

We now enumerate some definitions and remarks specifically referred to later on.

2.1. Definition. We say $A$ and $B$ are disjoint if and only if $A B=0$. We say a family $\mathfrak{F}$ of sets is disjointed if and only if $A B=0$ whenever $A$ and $B$ are different members of $\mathfrak{F}$.

2.2. Definition. We say $A$ and $B$ are semi-disjoint if and only if neither is a subset of the other. We say a family $\mathfrak{F}$ is semi-disjointed if and only if no member of $\mathfrak{F}$ is a subset of a different member.

2.3. Definition. The family $\mathfrak{U}$ is defined by $\phi \in \mathfrak{U}$ if and only if $\phi$ is a function on $\varepsilon_{2}$ to $E_{\imath}[0 \leqq t \leqq \infty]$ such that:

I. $\phi(0)=0$;

II. $\phi(B) \leqq \phi(A)$ whenever $B \subset A \subset E_{2}$;

III. If $\mathfrak{F}$ is a countable subfamily of $\varepsilon_{2}$, then

$$
\phi[\sigma(\mathfrak{F})] \leqq \sum_{\mathcal{S} \in \mathfrak{F}} \phi(s) ;
$$

IV. $\phi(A+B)=\phi(A)+\phi(B)$ whenever dist $(A, B)>0$.

2.4. Definition. The family $\mathfrak{M}$ is defined by $\phi \in \mathfrak{M}$ if and only if $\phi \in \mathfrak{U}$ and $\phi\left(E_{2}\right)<\infty$.

Due to Carathéodory is the following

2.5. Definition. We say $A$ is $\phi$ measurable if and only if $A \subset E_{2}, \phi \in \mathfrak{U}$,

$$
\phi(W)=\phi(W A)+\phi(W \tilde{A})
$$

for each set $W \subset E_{2}$.

2.6. Definition. For $0<\rho$ we define $L_{\rho}$ as the function on $\varepsilon_{2}$ such that $A \subset E_{2}$ implies $L_{p}(A)$ is the infimum of numbers of the form

$$
\sum_{s \in \leftrightarrow}(\operatorname{diam} s)
$$

where $(S)$ is such a countable family of nonvacuous open subsets of $E_{2}$ that 
and

$$
\sigma(अ) ~ ? A
$$

$$
\operatorname{diam} s<\rho \text { whenever } s \in \mathbb{B} \text {. }
$$

2.7. Definition. We define $E$ as the function on $\varepsilon_{2}$ such that $A \subset E_{2}$ implies

$$
L(A)=\lim _{\rho \rightarrow 0+} L_{\rho}(A) .
$$

Definitions 2.6 and 2.7 are due to Carathéodory [2]. The number $L(A)$ is commonly known as the Carathéodory linear measure of $A$.

2.8. Definition. By counting measure we mean the function $\phi$ on $\varepsilon_{2}$ such that $\phi(s)$ is the number of points in $s$ whenever $s \subset E_{2}$.

2.9. Abbreviation. We use the symbol $\boldsymbol{\Omega}_{x}^{r}$ as an abbreviation for

$$
E_{2} \underset{y}{E}[|y-x|<r]
$$

and the symbol $\mathfrak{\mho}_{x}^{r}$ as an abbreviation for

$$
E_{2} \underset{y}{E}[|y-x| \leqq r] \text {. }
$$

2.10. Definition. A set $\Gamma$ will be called a circle if and only if there is an $x \in E_{2}$ and a number $r$ with $0<r<\infty$ such that $\Gamma=\Re_{x}^{r}$.

2.11. Definition. For $\phi \in \mathfrak{U}, A \subset E_{2}$ and $x \in E_{2}$ we define

$$
\begin{aligned}
& \mathfrak{D}_{\phi}^{\nabla}(A, x)=\lim _{r \rightarrow 0+} \frac{\phi\left(A \Omega_{x}^{r}\right)}{2 r}, \\
& \mathfrak{D}_{\phi}^{\Delta}(A, x)=\varlimsup_{r \rightarrow 0+} \frac{\phi\left(A \mathfrak{\Omega}_{x}^{r}\right)}{2 r}, \\
& \mathfrak{D}_{\phi}^{\Delta}(A, x)=\varlimsup_{(\operatorname{diam} \Gamma) \rightarrow 0, \Gamma \in \mathfrak{D}} \frac{\phi(A \Gamma)}{(\operatorname{diam} \Gamma)},
\end{aligned}
$$

where $\mathfrak{S}=E_{\Gamma}[\Gamma$ is a circle and $x \in \Gamma]$ and call $\mathfrak{D}_{\phi}^{\nabla}(A, x)$ and $\mathfrak{D}_{\phi}^{\Delta}(A, x)$ respectively the lower and upper $\phi$ density of $A$ at $x$.

2.12. Definition. For $\phi \in \mathfrak{U}$ and $x \in E_{2}$ we define

$$
\mathfrak{D}_{\phi}^{\nabla}(x)=\mathfrak{D}_{\phi}^{\nabla}\left(E_{2}, x\right), \mathfrak{D}_{\phi}^{\Delta}(x)=\mathfrak{D}_{\phi}^{\Delta}\left(E_{2}, x\right) \text { and } \mathfrak{D}_{\phi}^{\Delta}(x)=\mathfrak{D}_{\phi}^{\Delta}\left(E_{2}, x\right) \text {. }
$$

2.13. Definitions. A set $A$ is said to be strictly rectifiable if and only if $A \subset E_{2}$ and there is: a number $M>0$; a closed interval $J$; a function $f$, with domain $J$ and range $A$, such that $f$ satisfies the Lipschitz condition

$$
\left|f\left(t^{\prime \prime}\right)-f\left(t^{\prime}\right)\right| \leqq M\left|t^{\prime \prime}-t^{\prime}\right| \quad \text { for } t^{\prime} \text { and } t^{\prime \prime} \text { in } J \text {. }
$$

2.14. Definition. A set $A$ is said to be rectifiable if and only if $A$ is a subset of a strictly rectifiable set. 
We shall use the obvious and elementary properties of rectifiable sets listed in

2.15. Remark. (i) If $A$ is rectifiable then $L(A)<\infty$.

(ii) Any subset of a rectifiable set is rectifiable.

(iii) If $\mathfrak{F}$ is a finite family of rectifiable sets then $\sigma(\mathfrak{F})$ is rectifiable.

(iv) If $A$ and $A^{\prime}$ are congruent and $A^{\prime}$ is rectifiable then $A$ is rectifiable.

(v) If $A$ is rectifiable then clsr $A$ is rectifiable.

2.16. Definition. A set $A$ is said to be $\phi$ rectifiable if and only if $A \subset E_{2}$, $\phi \in \mathfrak{U}$ and corresponding to each $\epsilon>0$ there is a strictly rectifiable set $B$ with $\phi(A \widetilde{B})<\epsilon$.

2.17. Definition. For $\phi \in \mathfrak{U}, A \subset E_{2}$ and $x \in E_{2}$ we define:

(i) $\operatorname{Sgn}(A, x)=\operatorname{clsr}\left[\Sigma_{y \in B}\{(y-x) /|y-x|\}\right]$ where $B=A-\{x\}$;

(ii) $\operatorname{dir}(A, x)=\prod_{\beta \in \mathfrak{F}} \operatorname{Sgn}(A \tilde{\beta}, x)$ where $\mathfrak{F}=E_{\beta}\left[\beta \Omega_{x}^{r}=0\right.$ for some $\left.r>0\right]$;

(iii) $\operatorname{dir}_{\phi}(A, x)=\prod_{\beta \in \Theta} \operatorname{Sgn}(A \widetilde{\beta}, x)$ where $\mathcal{S}=E_{\beta}\left[\mathfrak{D}_{\phi}^{\Delta}(\beta, x)=0\right]$.

2.18. Definition. For $\phi \in \mathfrak{U}$ and $x \in E_{2}$ we define

$$
\operatorname{dir}_{\phi}(x)=\operatorname{dir}_{\phi}\left(E_{2}, x\right)
$$

2.19. Definition. A set is said to be diametral if and only if it is of the form $\{-z\}+\{z\}$ where $z \in E_{2}$ and $|z|=1$.

A set is said to be nondiametral if and only if it is a subset of $E_{2}$ and is not diametral.

2.20. Definition. We say $A$ is directional at $x$ if and only if $\operatorname{dir}(A, x)$ is diametral.

2.21. Definition. We say $A$ is $\phi$ directional at $x$ if and only if $\operatorname{dir}_{\phi}(A, x)$ is diametral.

2.22. Definition. We say $A$ is restricted at $x$ if and only if there is a point $z$ in $E_{2}$ with $|z|=1$ for which $\{z\} \operatorname{dir}(A, x)=0$.

2.23. Remark. If $A \subset B$ and $B$ is restricted at $x$ then so is $A$.

2.24. Definition. We say $A$ is $\phi$ restricted at $x$ if and only if

$$
\mathfrak{D}_{\phi}^{\Delta}(A, x)>0
$$

and there is a diametral set $Z$ for which

$$
Z \operatorname{dir}_{\phi}(A, x)=0 \text {. }
$$

2.25. Abbreviation. We employ $\hat{A}$ as an abbreviation for

$$
E_{2} \underset{x}{E}[|x-y| \leqq 2 \operatorname{diam} A \text { for some } y \text { in } A] \text {. }
$$

2.26. Remark. We note that $\hat{\Gamma}=\Omega_{x}^{s r}$ whenever $\Gamma=\Omega_{x}^{r}$.

2.27. Abbreviation. We use $p * q$ as an abbreviation for

$$
\Omega_{p}^{|p-q|} \Omega_{q}^{|p-q|} \text {. }
$$

3. Measure. Consulting 2.3, 2.4, 2.5 for nomenclature we recall certain 
general properties of measure.

If $A$ is $\phi$ measurable and $W$ and $B$ are arbitrary subsets of $E_{2}$, then

$$
\phi(W A+W B)+\phi(W A B)=\phi(W A)+\phi(W B) .
$$

If $A$ is $\phi$ measurable and $\phi(B)<\infty$ then

$$
\phi(B-A)=\phi(B)-\phi(B A) .
$$

If $\mathfrak{F}$ is a countable family of $\phi$ measurable sets then $\sigma(\mathfrak{F})$ is $\phi$ measurable. If $A$ is $\phi$ measurable then $\tilde{A}$ is $\phi$ measurable.

If $W \subset E_{2}$ and $\mathfrak{F}$ is a countable disjointed family $\phi$ of measurable sets then

$$
\phi\left(\sum_{S \in \mathfrak{F}} W S\right)=\sum_{s \in \mathfrak{F}} \phi(W S) .
$$

If $B$ is a Borel set and $\phi \in \mathfrak{U}$ then $B$ is $\phi$ measurable.

Several comments, which may incidentally help explain some peculiarities of certain subsequent proofs, are incorporated in

3.1. RemarK. Suppose $A_{1} \subset A_{2} \subset A_{3} \subset \cdots$ with $A=\sum_{n-1}^{\infty} A_{n} \subset E_{2}$.

If $\phi \in \mathfrak{U}, W$ is an arbitrary subset of $E_{2}$, and $A_{n}$ is $\phi$ measurable for each positive integer $n$, then as is well known

$$
\phi(W A)=\lim _{n \rightarrow \infty} \phi\left(W A_{n}\right) .
$$

Furthermore if $\phi \in \mathfrak{U}$ and for each positive integer $n_{;} \cdot A_{n}$ is contained in a $\phi$ measurable set with the same $\phi$ measure as $A_{n}$, then

$$
\phi(A)=\lim _{n \rightarrow \infty} \phi\left(A_{n}\right) .
$$

However, even with $\phi \in \mathfrak{M}$, it may happen that

$$
\lim _{n \rightarrow \infty} \phi\left(A_{n}\right)<\phi(A),
$$

as may be shown by an example. Accordingly certain techniques, available to us in handling such a measure (see 2.6 and 2.7) as $L$, are unemployable in dealing with a general measure $\phi$ in $\mathfrak{M}$ or $\mathfrak{U}$. Nevertheless if $\phi \in \mathfrak{U}$ and $\phi(A)>0$ it is obvious from 2.3 III that

$$
\lim _{n \rightarrow \infty} \phi\left(A_{n}\right)>0 \text {. }
$$

We state without proot

3.2. TheOREM. If $\phi \in \mathfrak{U}, A \subset E_{2}$, and $\Phi$ is the function on $\varepsilon_{2}$ such that $\Phi(s)=\phi(A s)$ for $s \subset E_{2}$, then $\Phi \in \mathfrak{U}$ and $s$ is $\Phi$ measurable whenever $s$ is $\phi$ meaisurable; furthermore if in addition $\phi(A)<\infty$, then $\Phi \in \mathfrak{M}$.

3.3. ClichÉ. If $\phi \in \mathfrak{U}$ and $\phi(A)<\infty$ and some statement involving $\phi$ and 
$A$ is readily proved by the introduction of the $\Phi$ of 3.2 , we indicate this state of affairs by the use of some phrase containing the words "intersecting measure."

Our first use of intersecting measure occurs in 3.7.

After referring to 2.6 and 2.7 it is easy to check the following well known

3.4. THEOREM. $L \in \mathfrak{U}$ and corresponding to each set $A \subset E_{2}$ there is a $G_{\delta}$ set $B$ for which

$$
A \subset B \text { and } L(A)=L(B) .
$$

In this section and throughout the paper the measure functions in which we are primarily interested are rather special in that they are on $\varepsilon_{2}$ and belong either to $\mathfrak{U}$ or its subclass $\mathfrak{M}$. Nevertheless the results of this section and their proofs are valid when we regard $E_{2}$ as an abstract metric space. In this sense 3.7 and 3.13 are apparently [7, Lemma 15.1, p. 152] heretofore unknown, the latter being an extension of a result, due to Hahn [3, Theorem IV, p. 447], which lessens the importance in measure theory of such special Borel sets as $G_{\S}$ 's.

The following lemma is easy to prove without transfinite induction (see $[4$, p. 159]) and is rather well known.

3.5. Lemma. $A$ set $A \subset E_{2}$ is a Borel set if and only if it is a member of every family $\mathfrak{F}$ such that:

(i) $B \in F_{\text {implies }} B \subset E_{2}$;

(ii) $B \in \mathfrak{F}$ whenever $B$ is a closed subset of $E_{2}$;

(iii) $B$ is a countable nonvacuous subfamily of $\mathfrak{F}$ implies

$$
\prod_{\mathfrak{A} \in \mathbb{S}} s \in \mathfrak{F} \text { and } \sum_{s \in \mathfrak{S}} s \in \mathfrak{F}
$$

We are indebted to Alfred Horn for pointing out that the use of the above lemma materially simplifies the proof of the following

3.6. Theorem. If $\phi \in \mathbb{M}, A$ is a Borel set, and $\epsilon>0$, then there is a closed subset $C$ of $A$ with $\phi(A \bar{C})<\epsilon$.

Proof. Let $\mathfrak{F}$ be the family defined by the conditions: $B \in \mathfrak{F}$ if and only if $B C E_{2}$ and corresponding to each number $\epsilon>0$ there is a closed set $C$ such that $\phi(B \tilde{C})<\epsilon$. The members of $\mathfrak{F}$ are therefore subsets of $E_{2}$ and clearly $B \in \mathfrak{F}$ whenever $B$ is a closed subset of $E_{2}$. Also $B \in \mathfrak{F}$ implies $B$ is $\phi$ measurable.

Let $A_{1}, A_{2}, A_{3}, \cdots$ be sets in $\mathfrak{F}$. Let $\eta>0$. For $n=1,2,3, \cdots$ choose a closed set $C_{n}$ such that

$$
C_{n} \subset A_{n}, \quad \phi\left(A_{n}-C_{n}\right)<\eta 2^{-n} .
$$

Thus 


$$
\prod_{j=1}^{\infty} A_{i}-\prod_{j=1}^{\infty} C_{j} \subset \sum_{j=1}^{\infty}\left(A_{j}-C_{j}\right)
$$

and

$$
\phi\left(\prod_{j=1}^{\infty} A_{j}-\prod_{j=1}^{\infty} C_{j}\right) \leqq \phi\left[\sum_{j=1}^{\infty}\left(A_{j}-C_{j}\right)\right] \leqq \sum_{j=1}^{\infty} \phi\left(A_{i}-C_{j}\right)<\eta
$$

where $\prod_{j-1}^{\infty} C_{j}$ is clearly a closed subset of $\prod_{j-1}^{\infty} A_{j}$. On the other hand

$$
\begin{aligned}
\lim _{n \rightarrow \infty} \phi\left(\sum_{j=1}^{\infty} A_{i}-\sum_{j=1}^{n} C_{j}\right) & =\phi\left(\sum_{j=1}^{\infty} A_{j}-\sum_{j=1}^{\infty} C_{j}\right) \leqq \phi\left[\sum_{j=1}^{\infty}\left(A_{j}-C_{j}\right)\right] \\
& \leqq \sum_{j=1}^{\infty} \phi\left(A_{i}-C_{j}\right)<\eta,
\end{aligned}
$$

and $n$ may be chosen so large that

$$
\phi\left(\sum_{j=1}^{\infty} A_{j}-\sum_{j=1}^{n} C_{j}\right)<\eta
$$

where $\sum_{j-1}^{n} C_{j}$ is of course a closed subset of $\sum_{j-1}^{\infty} A_{j}$. Accordingly the definition of $\mathfrak{F}$, the arbitrariness of $\eta,(1)$, and (2), combine to tell us that

$$
\sum_{j=1}^{\infty} A_{i} \in \mathfrak{F}, \quad \prod_{j=1}^{\infty} A_{j} \in \mathfrak{F} .
$$

Thus using 3.5 we see that each Borel set is a member of $\mathfrak{F}$.

The theorem now follows from the definition of $\mathfrak{F}$.

3.7. Theorem. If $\phi \in \mathfrak{U}, A$ is a Borel set with $\phi(A)<\infty$, and $\epsilon>0$, then there is a closed subset $C$ of $A$ with $\phi(A \tilde{C})<\epsilon$.

Proof. In the spirit of 3.3 we introduce intersecting measure; that is we let $\Phi$ be the function on $\varepsilon_{2}$ such that

$$
\Phi(s)=\phi(A s) \text { for } s \subset E_{2},
$$

invoke 3.2, note that $\Phi \in \mathbb{M}$ and $\Phi(A \tilde{C})=\phi(A \tilde{C})$ for $C \subset E_{2}$ and use 3.6.

3.8. Corollary. If $\phi \in \mathfrak{U}$ and $A$ is a Borel set with $\phi(A)<\infty$, then $A$ is

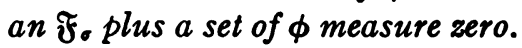

3.9. Corollary. If $\phi \in \mathfrak{U}, \psi \in \mathfrak{U}, B$ is a Borel set, $\phi(B)+\psi(B)<\infty$, and if $\phi(C)=\psi(C)$ for each closed set $C \subset B$, then $\phi(B)=\psi(B)$.

3.10. Theorem. If $\phi \in \mathfrak{M}, \psi \in \mathfrak{M}$ and $\phi(C)=\psi(C)$ for each closed set $C$, then $\phi(B)=\psi(B)$ for each Borel set $B$.

Proof. This theorem is an immediate consequence of Corollary 3.9. 
3.11. Corollary. If $\phi \in \mathfrak{M}, \psi \in \mathfrak{M}$, and $\phi(G)=\psi(G)$ for each open set $G$, then $\phi(B)=\psi(B)$ for each Borel set $B$.

3.12. THEOREM. If $\phi \in \mathfrak{U}$ and $\psi$ is the function on $\varepsilon_{2}$ such that $s \subset E_{2}$ implies $\psi(s)$ is the infimum of numbers of the form $\phi(S)$ where $S$ is an open set containing $s$, then $\psi \in \mathfrak{U}$; furthermore if in addition $\phi \in \mathfrak{M}$, then $\psi \in \mathbb{M}$ and $\phi(B)=\psi(B)$ for each Borel set $B$.

Proof. Clearly $\psi \in \mathfrak{U}$ whenever $\phi \in \mathfrak{U}$. We let $\phi \in \mathfrak{M}$, make a simple check to show that $\psi \in \mathfrak{M}$, note that $\phi(G)=\psi(G)$ for each open set $G$ and apply Corollary 3.11 .

3.13. TheOREM. If $\phi \in \mathfrak{U}, \epsilon>0, \phi(\alpha)<\infty, \alpha$ is $\phi$ measurable, and if each subset $A$ of $E_{2}$ is contained in a Borel set with the same $\phi$ measure, then there is $a$ closed set $\beta$ such that $\beta \subset \alpha$ and $\phi(\alpha \tilde{\beta})<\epsilon$.

Proof. Let $\beta_{1}$ be a Borel set containing $\alpha$ with the same $\phi$ measure as $\alpha$. Since $\phi\left(\beta_{1} \tilde{\alpha}\right)=0$ we may inclose $\beta_{1} \tilde{\alpha}$ in a Borel set of $\phi$ measure zero and subtract this set from $\beta_{1}$, in order to obtain a Borel set $\beta_{2}$ with $\beta_{2} \subset \alpha$ and $\phi(\alpha)=\phi\left(\beta_{1}\right)=\phi\left(\beta_{2}\right)$. Application of Corollary 3.7 to the Borel set $\beta_{2}$ completes the proof.

A glance at 2.8 makes easy the proof of

3.14. THEOREM. If $\phi$ is counting measure, then $\phi \in \mathfrak{U}$ and every subset of $E_{2}$ is $\phi$ measurable.

4. Connected sets. Recalling the Definition 2.2 of a semi-disjointed family we state and prove

4.1. THEOREM. If $\mathfrak{F}$ is any family of sets and

$$
\mathfrak{F} \underset{S}{E}[s \subset S]
$$

is a finite family whenever $s \in \mathfrak{F}$, then there is a semi-disjointed subfamily $\mathbb{H}$ of. $\mathfrak{F}$ such that

$$
\sigma(अ)=\sigma(\mathfrak{F})
$$

Proof. Let

$$
\mathbb{B}=\mathfrak{F} E[s \subset s \in \mathfrak{F} \text { implies } s=S]
$$

It is obvious that $B$ is semi-disjointed and from the hypothesis it is readily checked that every member of $\mathfrak{F}$ is: a subset of some set in $\$($ Consequently

$$
\sigma(\mathfrak{F}) \subset \sigma(\mathfrak{S}) \subset \sigma(\mathfrak{F})
$$

4.2. ThEOREM. If $A$ is a connected set, $x \in A$, and $0 \leqq r \leqq 2^{-1}$ (diam $A$ ), then $r \leqq L\left(A \Omega_{x}^{r}\right)$. 
Proof. Since $A$ is connected and $0 \leqq r \leqq 2^{-1}$ (diam $A$ ) it follows that $0 \leqq r^{\prime}<r$ implies there is a $y \in A$ for which $|y-x|=r^{\prime}$. Let $\mathfrak{F}$ be any countable family of nonvacuous open sets such that $\sigma(\mathfrak{F}) \supset A \Omega_{x}^{r}$. For $s \in \mathfrak{F}$ let $M(s)$ and $m(s)$ be the supremum and infimum respectively of $\sum_{y \in s}\{|y-x|\}$ and note that (diam $s) \geqq M(s)-m(s)$. Clearly $0 \leqq r^{\prime}<r$ implies there is an $s \in \mathfrak{F}$ for which $m(s) \leqq r^{\prime} \leqq M(s)$, and therefore

$$
\sum_{: \in \Im}(\operatorname{diam} s) \geqq \sum_{: \in \Im}[M(s)-m(s)] \geqq r .
$$

Consequently, the arbitrariness of $\mathfrak{F}$ guarantees $L\left(A \Omega_{x}^{r}\right) \geqq r$.

For use in the next theorem and its proof the number $T_{\alpha}^{\beta} f$ is defined, whenever $-\infty<\alpha<\beta<\infty$ and $f$ is a function whose domain includes $[\alpha, \beta]$ and whose range is contained in $E_{1}$, as the supremum of numbers of the form

$$
\sum_{j=1}^{N}\left|f\left(t_{j}\right)-f\left(t_{j-1}\right)\right|
$$

where $N$ is a positive integer and $\alpha=t_{0}<t_{1}<t_{2}<\cdots<t_{N}=\beta$.

We read $T_{\alpha}^{\beta} f$ as the total variation from $\alpha$ to $\beta$ of $f$ and recall, incidentally, that $L(S)=T_{\alpha}^{\beta} f$ whenever $f$ is a continuous univalent function whose domain is the closed interval $[\alpha, \beta]$ and whose range is $S \subset E_{2}$.

4.3. THEOREM. If $\mathfrak{F}$ is a nonvacuous finite semi-disjointed family of $n$ circles with $\sigma(\mathfrak{F})$ connected, then there is a number $a>0$ and a function $f$ such that:

(i) the domain of $f$ is $[0, a]$;

(ii) the range of $f$ is $\sum_{s \in \mathfrak{F}}($ bndr $s$ );

(iii) $f$ satisfies a Lipschitz condition on $[0, a]$;

(iv) $T_{0}^{a} f=\pi \sum_{s \in \mathfrak{F}}(\operatorname{diam} s)$.

Proof. Assume the theorem is not true and let $k$ be the smallest value of $n$ for which the theorem is false. Since the theorem is obvious if $n=1$ we see that $k \geqq 2$.

Let $\$(S)$ be a semi-disjointed family of $k$ circles, with $\sigma(\mathbb{S})$ connected, corresponding to which there is no function of the required type.

Since $\sigma(\mathbb{S})$ is open and connected, it cannot be split into two non-empty open sets without points in common. Accordingly we select a circle of $\&$ and next select a different circle of $\mathbb{B}$ which intersects the first circle and proceeding step by step arrive at a family $\mathfrak{W}$ of $k-1$ circles of $(\mathbb{S}$ such that $\sigma(\mathfrak{Q})$ is connected. Letting $s_{0}$ be the sole member of $\mathfrak{B}-\mathfrak{W}$, we use the definition of $k$ to ascertain a positive number $a_{0}$ and a function $f_{0}$ such that:

(1) the domain of $f_{0}$ is $\left[0, a_{0}\right]$;

(2) the range of $f_{0}$ is $\sum_{s \in \mathfrak{S}}($ bndr $s)$;

(3) $f_{0}$ satisfies a Lipschitz condition;

(4) $T_{0}^{a_{0}} f_{0}=\pi \sum_{s \in \mathfrak{\Phi}}(\operatorname{diam} s)$ :

Since $\sigma(\mathfrak{F})$ is connected, the set $s s_{0}$ is nonvacuous for some $s \in \mathfrak{E}$; and, 
since $s_{0}$ and this $s$ are semi-disjoint, the intersection of their boundaries is also nonvacuous. In view of this we select a point $z_{0}$ such that

$$
z_{0} \in\left(\text { bndr } s_{0}\right) \sum_{s \in \mathfrak{F}}(\text { bndr } s)
$$

and choose $t_{0}$ so that $0 \leqq t_{0} \leqq a_{0}$ and $f_{0}\left(t_{0}\right)=z_{0}$. Let $x_{0}$ and $r_{0}$ be such that $s_{0}=\Re_{x_{0}}^{r_{0}}$, take $\alpha$ in $E_{1}$ so that $z_{0}-x_{0}=r_{0} e^{i \alpha}$, and define the function $f$ on $\left[0, a_{0}+2 \pi\right]$ so that

$$
f(t)=\left[\begin{array}{ll}
f_{0}(t) & \text { for } 0 \leqq t \leqq t_{0}, \\
x_{0}+r_{0} e^{i\left(\alpha+t-t_{0}\right)} & \text { for } t_{0}<t<t_{0}+2 \pi, \\
f_{0}(t-2 \pi) & \text { for } t_{0}+2 \pi \leqq t \leqq a_{0}+2 \pi .
\end{array}\right.
$$

A check shows this function $f$ corresponds to $\&$ as prescribed by our theorem, in contradiction to our previous statement that there was no such function.

By using the device known as parameterizing according to arc length we first obtain the

4.4. Corollary. If $\mathfrak{F}$ is a nonvacuous finite semi-disjointed family of open circles with $\sigma(\mathfrak{F})$ connected and if $l=\pi \sum_{\mathfrak{s} \in \mathfrak{F}}(\operatorname{diam} s)$, then there is a function $f$ on $[0, l]$ with range $\sum_{s \in \mathfrak{F}}($ bndr $s)$ such that

$$
\left|f\left(t^{\prime \prime}\right)-f\left(t^{\prime}\right)\right| \leqq\left(t^{\prime \prime}-t^{\prime}\right) \quad \text { for } 0 \leqq t^{\prime} \leqq t^{\prime \prime} \leqq l .
$$

Next by a transformation we obtain the

4.5. Corollary. If $\mathfrak{F}$ is a nonvacuous finite semi-disjointed family of open circles with $\sigma(\mathfrak{F})$ connected and if $l=\pi \sum_{s \in \mathfrak{F}}(\operatorname{diam} s)$, then there is a function $f$ on $[0,1]$ with range $\sum_{s \in \mathfrak{F}}($ bndr $s)$ such that

$$
\left|f\left(t^{\prime \prime}\right)-f\left(t^{\prime}\right)\right| \leqq l\left(t^{\prime \prime}-t^{\prime}\right) \quad \text { for } 0 \leqq t^{\prime} \leqq t^{\prime \prime} \leqq 1 .
$$

4.6. THEOREM. If $A \subset E_{2}$ is a nonvacuous continuum with $L(A)<\infty$ then $A$ is strictly rectifiable.

Proof. We infer from 4.2 that $A$ is bounded, and, relying on the fact that a bounded open set of diameter $d$ may be included in a circle of diameter $2 d$, we use Definition 2.7 of $L$ and the Heine-Borel theorem to secure, for each positive integer $n$, a finite family $\mathfrak{F}_{n}$ of circles such that

$$
A \subset \sigma\left(\mathfrak{F}_{n}\right), \quad \sum_{S \in \mathfrak{F}_{n}}(\operatorname{diam} S)<2 L(A)+1
$$

and such that, in addition,

$$
\operatorname{diam} s<1 / n, \quad s A \neq 0 \quad \text { for } s \in \mathfrak{F}_{n} .
$$

Now for each positive integer $n$ we select from $\mathfrak{F}_{n}$, according to Theorem 4.1, a semi-disjointed subfamily $\mathbb{S}_{n}$ covering $A$; note that $s \in \mathbb{S}_{n}$ implies 


$$
\sum_{s \in G_{n}}(\operatorname{diam} S)<2 L(A)+1, \quad \operatorname{diam} s<1 / n, \quad s A \neq 0 ;
$$

and, employing the connectedness of $A$, infer that $\sigma\left(\Theta_{n}\right)$ is likewise connected. Upon setting

$$
\lambda=\pi(2 L(A)+1)
$$

we are now justified by 4.5 in ascertaining a sequence $f$ such that: corresponding to each positive integer $n, f_{n}$ is a function on $[0,1]$; its range is $\sum_{s \in \Theta_{n}}($ bndr $S$ ); and it satisfies the Lipschitz condition

$$
\left|f_{n}\left(t^{\prime \prime}\right)-f_{n}\left(t^{\prime}\right)\right| \leqq \lambda\left(t^{\prime \prime}-t^{\prime}\right) \quad \text { for } 0 \leqq t^{\prime} \leqq t^{\prime \prime} \leqq 1 .
$$

Thus using the equi-continuity principle found, for example, in Theorem $V$ on page 304 of [3], we extract a uniformly convergent subsequence $g$ of $f$, and denote the limit function by $\bar{g}$. Clearly

$$
\left|\bar{g}\left(t^{\prime \prime}\right)-\bar{g}\left(t^{\prime}\right)\right| \leqq \lambda\left(t^{\prime \prime}-t^{\prime}\right) \quad \text { whenever } 0 \leqq t^{\prime} \leqq t^{\prime \prime} \leqq 1,
$$

and a check reveals

$$
A=\sum_{t \in[0,1]}\{\bar{g}(t)\} .
$$

Consequently $A$ is strictly rectifiable according to 2.13 .

4.7. LeMMA. If $C$ is a continuum and $\mathfrak{F}$ is a semi-disjointed family of circles such that

(i) $\sigma(\mathfrak{F}) \subset C$,

(ii) $\epsilon>0$ implies $\mathfrak{F} E_{S}[\operatorname{diam} S>\epsilon]$ is finite, then

$$
D=[C-\sigma(\mathfrak{F})]+\sum_{s \in \mathfrak{F}}(\operatorname{bndr} S)
$$

is a continuum.

Proof. We first prove $D$ is closed. Let $p_{0}$ be a cluster point of

$$
\sum_{s \in \mathfrak{F}}(\operatorname{bndr} S)
$$

which is not a point of the closed set $C-\sigma(\mathfrak{F})$. Since $C$ is closed, $p_{0}$ is a point of the open set $\sigma(\mathfrak{F})$ and we let $S_{0} \in \mathfrak{F}$ be such that $p_{0} \in S_{0}$ and note that

$$
\delta=\operatorname{dist}\left(\left\{p_{0}\right\}, \tilde{S_{0}}\right)
$$

is positive. Letting

$$
\mathfrak{W}=\mathfrak{F} \underset{\mathbb{S}}{E}\left[(\operatorname{bndr} S) \Re_{p_{0}}^{\delta / 2} \neq 0\right]
$$

we see from the semi-disjointedness of $\mathfrak{F}$ that $S \Omega_{p_{0}}^{\delta / 2} \neq 0$ and $S \widetilde{S}_{0} \neq 0$ whenever 
$S \in \mathfrak{W}$. Accordingly each member of $\mathfrak{W}$ has diameter greater than $2^{-1} \delta$ and thus from (ii) it is clear that $\mathfrak{E}$ is finite. Therefore $p_{0}$ is a cluster point of the closed set $\sum_{s \in \mathfrak{\$}}($ bndr $S)$ with

$$
p_{0} \in \sum_{s \in \mathfrak{F}}(\text { bndr } S) \subset \sum_{s \in \mathfrak{F}}(\text { bndr } S) \subset D .
$$

Hence $D$ is closed.

Next the set $D$ is connected as we prove by contradiction. Suppose $D=D_{1}+D_{2}$ where $D_{1}$ and $D_{2}$ are non-empty closed sets and $D_{1} D_{2}=0$.

We define

$$
\mathfrak{F}_{1}=\mathfrak{F}\left[(\text { bndr } s) \subset D_{1}\right], \quad \mathfrak{F}_{2}=F_{S}^{E}\left[(\text { bndr } S) \subset D_{2}\right]
$$

Since the boundary of a circle is connected we have

$$
\mathfrak{F}=\mathfrak{F}_{1}+\mathfrak{F}_{2} \text {. }
$$

Moreover

$$
\sigma\left(\mathfrak{F}_{1}\right) \sigma\left(\mathfrak{F}_{2}\right)=\sum_{s \in \mathfrak{F}_{1}} \sum_{s \in \mathfrak{F}_{2}} s S=0
$$

because the boundaries of two semi-disjoint intersecting circles intersect. Using the definition of $D$ we conclude

$$
\begin{aligned}
\sigma\left(\mathfrak{F}_{2}\right) D_{1} & =\sigma\left(\mathfrak{F}_{2}\right) D_{1} \sum_{s \in \mathfrak{F}}(\text { bndr } s)=\sigma\left(\mathfrak{F}_{2}\right) \sum_{s \in \mathfrak{F}}\left[D_{1}(\text { bndr } s)\right] \\
& =\sigma\left(\mathfrak{F}_{2}\right) \sum_{, \in \mathfrak{F}_{1}}(\text { bndr } s)=\sum_{s \in \mathfrak{\Im}_{2}} \sum_{s \in \mathfrak{F}_{1}} S(\text { bndr } s)=0 ;
\end{aligned}
$$

the last step follows from (2) and the fact that two circles intersect whenever one circle intersects the boundary of the other. By symmetry

$$
\sigma\left(\mathfrak{F}_{2}\right) D_{1}=\sigma\left(\mathfrak{F}_{1}\right) D_{2}=0 .
$$

With the aid of (ii) and the definitions of $\mathfrak{F}_{1}$ and $\mathfrak{F}_{2}$ we check that the sets

$$
D_{1}+\sigma\left(\mathfrak{F}_{1}\right) \text { and } D_{2}+\sigma\left(\mathfrak{F}_{2}\right)
$$

are closed and utilize (1), (2), and (3) to see that their intersection, namely

$$
D_{1} D_{2}+\sigma\left(\mathfrak{F}_{1}\right) D_{2}+\sigma\left(\mathfrak{F}_{2}\right) D_{1}+\sigma\left(\mathfrak{F}_{1}\right) \sigma\left(\mathfrak{F}_{2}\right),
$$

is vacuous. Consequently

$$
C=D+\sigma(\mathfrak{F})=\left[D_{1}+\sigma\left(\mathfrak{F}_{1}\right)\right]+\left[D_{2}+\sigma\left(\mathfrak{F}_{2}\right)\right]
$$

and thus $C$ is disconnected in contradiction to the hypothesis that $C$ is a continuum.

5. Density theory. That the use of $\Omega_{x}^{r}$ rather than $\mathfrak{C}_{x}^{r}$ in density definitions (see 2.11) is a convenience rather than a necessity is pointed out in the following 
5.1. REMARK. If $\phi \in \mathfrak{U}, A \subset E_{2}$, and $x \in E_{2}$, then

$$
\begin{gathered}
\mathfrak{D}_{\phi}^{\nabla}(A, x)=\lim _{r=0+} \frac{\phi\left(A \mathfrak{C}_{x}^{r}\right)}{2 r}, \quad \mathfrak{D}_{\phi}^{\Delta}(A, x)=\varlimsup_{r \rightarrow 0+} \frac{\phi\left(A \mathfrak{C}_{x}^{r}\right)}{2 r}, \\
\mathfrak{D}_{\phi}^{\Delta}(A, x)=\varlimsup_{(\operatorname{diam} \Gamma) \rightarrow 0, \Gamma \in \mathfrak{Q}} \frac{\phi(A \Gamma)}{\operatorname{diam} \Gamma}
\end{gathered}
$$

where $\mathfrak{S}=E_{\Gamma}\left[x \in \Gamma=\mathfrak{G}_{y}^{r}\right.$ for some $y \in E_{2}$ and some $\left.r>0\right]$.

5.2. REMARK. Let $\phi \in \mathfrak{M}$. For each positive integer $n$, let $\Delta_{n}^{\nabla}, \Delta_{n}^{\Delta}$ and $\Delta_{n}^{\Delta}$ be the functions on $E_{2}$ such that $x \in E_{2}$ implies

$$
\begin{aligned}
\Delta_{,}^{\nabla}(x) & =\inf _{0<r<1 / n} \frac{\phi\left(\Omega_{x}^{r}\right)}{2 r}, \\
\Delta_{n}^{\Delta}(x) & =\sup _{0<r<1 / n} \frac{\phi\left(\Omega_{x}^{r}\right)}{2 r}, \\
\Delta_{n}^{\Delta}(x) & =\sup _{\Gamma \in \mathfrak{S}_{0} \operatorname{diam} \Gamma<1 / n} \frac{\phi(\Gamma)}{\operatorname{diam} \Gamma}, \text { where } \\
\mathfrak{S} & =\underset{\Gamma}{E}[\Gamma \text { is a circle and } x \in \Gamma],
\end{aligned}
$$

and note that the first of these functions is upper semi-continuous while the other two are lower semi-continuous and thus all three are Borel functions.

Also let $\Delta^{\nabla}, \Delta^{\Delta}$, and $\Delta^{\mathbf{\Delta}}$ be the functions on $E_{2}$ such that $x \in E_{2}$ implies

$$
\Delta^{\nabla}(x)=\lim _{n \rightarrow \infty} \Delta_{n}^{\nabla}(x), \quad \Delta^{\Delta}(x)=\lim _{n \rightarrow \infty} \Delta_{n}^{\Delta}(x), \quad \Delta^{\Delta}(x)=\lim _{n \rightarrow \infty} \Delta_{n}^{\Delta}(x) .
$$

We note that these functions are likewise Borel functions and moreover that for $x \in E_{2}$

$$
\Delta^{\nabla}(x)=\mathfrak{D}_{\phi}^{\nabla}(x), \Delta^{\Delta}(x)=\mathfrak{D}_{\phi}^{\Delta}(x) \text { and } \Delta^{\Delta}(x)=\mathfrak{D}_{\phi}^{\Delta}(x) .
$$

Thus Lemma 5.3 below is established.

The proofs of Lemmas 5.4 through 5.10 involve for the most part merely certain well known uniformization principles. The reader will presumably wish to make use of 3.1(i) in verifying Lemma 5.6. Lemmas 5.7 and 5.8 are corollaries of 5.6. Also leaving to the reader the proofs of Lemmas 5.5 and 5.10, we now suggest proofs for Lemmas 5.4 and 5.9.

If $\epsilon>0$ and $\mathfrak{D}_{\phi}^{\Delta}(x)>0$ for $\phi$ almost all $x$, then for $n$ sufficiently large the bounded Borel set

$$
\underset{x}{E}\left[|x|<n \text {. and } \mathfrak{D}_{\phi}^{\Delta}(x) \geqq 1 / n\right]
$$

not only has the $\phi$ measure of its complement less than $\epsilon$, but, by Theorem 
3.6, contains a closed subset $C$, necessarily bounded, such that $\phi(\widetilde{C})<\epsilon$. Thus Lemma 5.4 which follows is proved.

We next indicate a proof of Lemma 5.9.

Letting $R$ be the set of all positive rational numbers and checking that

$$
\underset{x}{E}\left[\mathfrak{D}_{\phi}^{\Delta}(x)<1.01 \mathfrak{D}_{\phi}^{\nabla}(x)\right]=\sum_{t \in R} E\left[t<\mathfrak{D}_{\phi}^{\nabla}(x) \leqq \mathfrak{D}_{\phi}^{\Delta}(x)<1.01 t\right],
$$

we choose a positive rational number $\alpha$ such that $\phi(B)>0$ where $B$ is the Borel set

$$
\underset{x}{E}\left[\alpha<\mathfrak{D}_{\phi}^{\nabla}(x) \leqq \mathfrak{D}_{\phi}^{\Delta}(x)<1.01 \alpha\right] .
$$

Accordingly, we select an integer $n$ so large that $\phi\left(B^{\prime}\right)>0$ where $B^{\prime}$ is the bounded Borel set

$$
B \underset{x}{E}\left[|x| \leqq n \text { and } \alpha<\Delta_{n}^{\nabla}(x) \leqq \Delta_{n}^{\Delta}(x)<1.01 \alpha\right] .
$$

Remembering Theorem 3.6, let $C$ be a bounded closed subset of $B^{\prime}$ with $\phi(C)>0$, let $\delta=n^{-1}$ and note that

$$
\alpha \leqq \phi\left(\Omega_{x}^{r}\right) / 2 r \leqq 1.01 \alpha<1.01 D_{\phi}^{\nabla}(x)
$$

for $x \in C$ and $0<r<\delta$.

With these remarks in mind the reader should have little difficulty in supplying proofs for Lemmas 5.5, 5.6 and 5.10.

5.3. LEMma. If $\alpha>0, \phi \in \mathfrak{M}$, then the three sets

$$
\underset{x}{E}\left[\mathfrak{D}_{\phi}^{\nabla}(x)>\alpha\right], \quad \underset{x}{E}\left[\mathfrak{D}_{\phi}^{\Delta}(x)>\alpha\right], \quad \underset{x}{E}\left[\mathfrak{D}_{\phi}^{\Delta}(x)>\alpha\right]
$$

are Borel sets.

5.4. LEMMA. If $\phi \in \mathfrak{M}, \epsilon>0$, and if $\mathfrak{D}_{\phi}^{\Delta}(x)>0$ for $\phi$ almost all $x$, then there is a number $\alpha>0$ and a bounded closed set $C$ with $\phi(\tilde{C})<\epsilon$ such that $\mathfrak{D}_{\phi}^{\Delta}(x)>\alpha$ for $x \in C$.

5.5. Lemma. If $\phi \in \mathfrak{M}, \alpha>0, \epsilon>0$, and if $\mathfrak{D}_{\phi}^{\nabla}(x)>\alpha$ for $\phi$ almost all $x$, then there is a number $\delta>0$ and a closed set $C$ with $\phi(\tilde{C})<\epsilon$ such that

$$
\alpha \leqq \phi\left(\Re_{x}^{r}\right) / 2 r
$$

for $x \in C$ and $0<r<\delta$.

5.6. LEмma. If $\phi \in \mathfrak{M}, \mu>0, \epsilon>0, A \subset E_{2}$, and $\mathfrak{D}_{\phi}^{\Delta}(x)<\mu$ for $\phi$ almost all $x$ in $A$, then there are positive numbers $\delta$ and $\lambda$ and a bounded closed set $C$ such that:

$$
\begin{gathered}
\lambda<\mu, \quad \phi(A \tilde{C})<\epsilon ; \\
\phi\left(\Omega_{x}^{r}\right) \leqq 2 \lambda r
\end{gathered}
$$

whenever $x \in A C$ and $0<r<\delta$. 
5.7. Lemma. If $\phi \in \mathfrak{M}, \epsilon>0$, and if $\mathfrak{D}_{\phi}^{\Delta}(x)<\infty$ for $\phi$ almost all $x$, then there is a finite number $\lambda>1$ and a bounded closed set $C$ with $\phi(\tilde{C})<\epsilon$ such that

$$
\phi\left(\Omega_{x}^{r}\right) \leqq 2 \lambda r
$$

for $x \in C$ and $0<r<\lambda^{-1}$.

5.8. LemMa. If $\phi \in \mathfrak{M}, \mu>0, \epsilon>0, A \subset E_{2}$, and $\mathfrak{D}_{\phi}^{\Delta}(x)<\mu$ for $\phi$ almost all $x \in A$, then there is a subset $B$ of $A$ and $a \delta>0$ such that

$$
\phi(B)>\phi(A)-\epsilon
$$

and

$$
\phi\left(\Omega_{x}^{r}\right) \leqq 2 \mu r
$$

for $x \in B$ and $0<r<\delta$.

5.9. Lemma. If $\phi \in \mathfrak{M}$ and $\mathfrak{D}_{\phi}^{\Delta}(x)<1.01 \mathfrak{D}_{\phi}^{\nabla}(x)$ for $\phi$ almost all $x$, then there are positive numbers $\alpha$ and $\delta$ and $a$ bounded closed set $C$ with $\phi(C)>0$ such that

$$
\alpha \leqq \frac{\phi\left(\Re_{x}^{r}\right)}{2 r} \leqq 1.01 \alpha<1.01 \mathfrak{D}_{\phi}^{\nabla}(x)
$$

for $x \in C$ and $0<r<\delta$.

5.10. LEMMA. If $\phi \in \mathfrak{M}$ and $3 \mathfrak{D}_{\phi}^{\Delta}(x)<4 \mathfrak{D}_{\phi}^{\nabla}(x)$ for $\phi$ almost all $x$, then there are positive numbers $\alpha, \delta$ and $\eta$, with $1<\eta<3^{-14}$, and a bounded closed set $C$. with $\phi(C)>0$, such that

$$
\alpha \leqq \phi\left(\Omega_{x}^{r}\right) / 2 r, \quad \phi(\Gamma) / \operatorname{diam} \Gamma \leqq \alpha \eta
$$

whenever $x \in C, \Gamma$ is a circle with $x \in \Gamma, 0<2 r<\delta$, diam $\Gamma<\delta$.

The next theorem, of which we make considerable use, is well known and follows immediately from Theorem 3.5 of [5]. Referring to 2.25 for the meaning of $\widehat{\text {, we state }}$

5.11. TheOREM. If $M>0$ and a set $A$ is covered by a family $\mathfrak{F}$ of circles each with diameter less than $M$, then there is a countable disjointed subfamily (\$) of F such that

$$
A \subset \sum_{\mathrm{r} \in \mathbb{\Theta}} \widehat{\Gamma}
$$

This Theorem 5.11 may also be proved directly, even though $A$ is unbounded, by a method closely patterned after the procedure outlined on p. 109 of [7].

5.12. TheOREM. If $\phi \in \mathfrak{M}$ and $A$ is a Borel set, then $\mathfrak{D}_{\phi}^{\Delta}(\tilde{A}, x)=0$ for $L$ almost all $x$ in $A$. 
Proof (by contradiction). Supposing the set

$$
A \underset{x}{E}\left[\mathfrak{D}_{\phi}^{\Delta}(\tilde{A}, x)>0\right]
$$

has positive $L$ measure, we select a positive number $\eta$ such that

$$
L(B)>0
$$

where

$$
B=\underset{x}{E}\left[\mathfrak{D}_{\phi}^{\Delta}(\tilde{A}, x)>\eta\right]
$$

Take $0<\epsilon<1$, choose a closed subset $C$ of $\tilde{A}$ such that $\phi(\tilde{A} \tilde{C})<\epsilon$, and let $\mathfrak{F}$ be the family of those circles of the form $\Omega_{x}^{r}$ where $x \in B$,

$$
0<r<\frac{\epsilon}{10} \frac{\operatorname{dist}(\{x\}, C)}{1+\operatorname{dist}(\{x\}, C)}, \quad \frac{\phi\left(\tilde{A} \Omega_{x}^{\top}\right)}{2 r}>\eta .
$$

With the help of Lemma 5.11 we pick a countable disjointed subfamily $\&$ of F such that

$$
B \subset \sum_{\mathrm{r} \in \Theta} \widehat{\Gamma}
$$

Noting diam $\Gamma<\epsilon$ and $\Gamma \subset \widetilde{C}$ for $\Gamma \in \mathfrak{F}$ we are assured by 2.6 and the definition of $\mathfrak{F}$ that

$$
\begin{aligned}
L_{\epsilon}(B) & \leqq \sum_{\Gamma \in \Theta}(\operatorname{diam} \widehat{\Gamma})=5 \sum_{\Gamma \in \Theta}(\operatorname{diam} \Gamma)<\frac{5}{\eta} \sum_{\Gamma \in \Theta} \phi(\tilde{A} \Gamma) \\
& =\frac{5}{\eta} \phi[\tilde{A} \sigma(\mathcal{S})] \leqq \frac{5}{\eta} \phi(\tilde{A} \tilde{C})<\frac{5}{\eta} \epsilon .
\end{aligned}
$$

From this last relation we have $L(B)=0$ in contradiction to (1). The proof is complete.

If $\Gamma$ is a circle of diameter $d$ and $x \in \Gamma$ then $\Gamma \subset \Omega_{x}^{d}$ and we have

5.13. Theorem. If $\phi \in \mathfrak{U}, A \subset E_{2}$, and $x \in E$; then

$$
\mathfrak{D}_{\phi}^{\hat{\Delta}}(A, x) / 2 \leqq \mathfrak{D}_{\phi}^{\Delta}(A, x) \leqq \mathfrak{D}_{\phi}^{\mathbf{A}}(A, x) .
$$

5.14. Theorem. If $\phi \in \mathfrak{M}$ and $A$ is a Borel set, then, for $L$ almost all $x$ in $A$,

$$
\mathfrak{D}_{\phi}^{\nabla}(x)=\mathfrak{D}_{\phi}^{\nabla}(A, x), \quad \mathfrak{D}_{\phi}^{\Delta}(x)=\mathfrak{D}_{\phi}^{\Delta}(A, x), \quad \mathfrak{D}_{\phi}^{\Delta}(x)=\mathfrak{D}_{\phi}^{\Delta}(A, x) .
$$

Proof. We check that

$$
\begin{aligned}
& \mathfrak{D}_{\phi}^{\nabla}(x) \leqq \mathfrak{D}_{\phi}^{\nabla}(A, x)+\mathfrak{D}_{\phi}^{\Delta}(\tilde{A}, x) \leqq \mathfrak{D}_{\phi}^{\nabla}(x)+\mathfrak{D}_{\phi}^{\Delta}(\tilde{A}, x), \\
& \mathfrak{D}_{\phi}^{\Delta}(x) \leqq \mathfrak{D}_{\phi}^{\Delta}(A, x)+\mathfrak{D}_{\phi}^{\Delta}(\tilde{A}, x) \leqq \mathfrak{D}_{\phi}^{\Delta}(x)+\mathfrak{D}_{\phi}^{\Delta}(\tilde{A}, x),
\end{aligned}
$$




$$
\mathfrak{D}_{\phi}^{\Delta}(x) \leqq \mathfrak{D}_{\phi}^{\mathbf{A}}(A, x)+\mathfrak{D}_{\phi}^{\mathbf{A}}(\tilde{A}, \dot{x}) \leqq \mathfrak{D}_{\phi}^{\Delta}(x)+2 \mathfrak{D}_{\phi}^{\Delta}(\tilde{A}, x),
$$

for $x \in E_{2}$.

Recourse to Theorem 5.12 completes the proof.

Using intersecting measure we obtain the

5.15. Corollary. If $\phi \in \mathfrak{U}, B \subset A \subset E_{2}, \phi(A)<\infty$, and $B$ is a Borel set, then, for $L$ almost all $x \in B$,

$$
\mathfrak{D}_{\phi}^{\nabla}(A, x)=\mathfrak{D}_{\phi}^{\nabla}(B, x), \quad \mathfrak{D}_{\phi}^{\Delta}(A, x)=\mathfrak{D}_{\phi}^{\Delta}(B, x), \quad \mathfrak{D}_{\phi}^{\Delta}(A, x)=\mathfrak{D}_{\phi}^{\Delta}(B, x) .
$$

5.16. THEOREM. If $A \subset E_{2}$ and $L(A)<\infty$, then $\mathfrak{D}_{L}(A, x) \leqq 1$ for $L$ almost all $x \in A$.

Proof (by contradiction). Assuming the set $A E_{x}\left[\mathfrak{D}_{\hat{L}}(A, x)>1\right]$ has positive $L$ measure, let $\eta>1$ be such that

$$
L(B)>0
$$

where

$$
B=A \underset{x}{E}\left[\mathfrak{D}_{L}^{\Delta}(A, x)>\eta\right] .
$$

Let $\epsilon$ be a positive number and use Definition 2.6 to ascertain a positive number $\delta$ such that both

$$
L(A) \leqq L_{\delta}(A)+\epsilon, \quad L(B) \leqq L_{\delta}(B)+\epsilon .
$$

Select a family $\mathfrak{F}$ of circles covering $B$ such that $\Gamma \in \mathcal{F}$ implies

$$
\operatorname{diam} \mathrm{\Gamma}<\delta / 5
$$

and

$$
L(A \Gamma)>\eta(\operatorname{diam} \Gamma) .
$$

Relying upon Theorem 5.11 we let $\mathfrak{B}$ be a countable disjointed subfamily of $\mathfrak{F}$ such that

$$
B \subset \sum_{r \in S} \widehat{\Gamma}
$$

and observe from (2) and Definition 2.6 not only that

$$
L_{\delta}(B) \leqq \sum_{\Gamma \in \Theta}(\operatorname{diam} \widehat{\Gamma})
$$

but also, upon setting $D=\sigma(())$, that

$$
L_{\delta}(A D) \leqq \sum_{\Gamma \in \mathcal{S}}(\operatorname{diam} \Gamma) .
$$

From Definitions 2.7 and 2.6 follow $0 \leqq L(A D)-L_{\delta}(A D), 0 \leqq L(A \tilde{D})$ $-L_{\delta}(A \tilde{D})$, and also 


$$
L_{\delta}(A D)+L_{\delta}(A \tilde{D}) \geqq L_{\delta}(A) ;
$$

whereas from the measurability of $D$ follows

$$
L(A D)+L(A \tilde{D})=L(A) .
$$

Consequently

$$
\begin{aligned}
0 \leqq L(A D)-L_{\delta}(A D) & \leqq\left[L(A D)-L_{\delta}(A D)\right]+\left[L(A \tilde{D})-L_{\delta}(A \tilde{D})\right] \\
& \leqq L(A)-L_{\delta}(A)<\epsilon
\end{aligned}
$$

and

$$
L(A D) \leqq L_{\delta}(A D)+\epsilon .
$$

Remembering the disjointedness of $B$ we have

$$
\begin{aligned}
\eta \sum_{\Gamma \in \Theta}(\operatorname{diam} \Gamma) & \leqq \sum_{\Gamma \in \Theta} L(A \Gamma)=L[A \sigma(\Theta)]=L(A D) \\
& \leqq L_{8}(A D)+\epsilon \leqq \sum_{\Gamma \in \Theta}(\operatorname{diam} \Gamma)+\epsilon,
\end{aligned}
$$

and therefore

$$
\sum_{r \in S}(\operatorname{diam} \Gamma) \leqq \frac{\epsilon}{\eta-1}
$$

Recapitulation now reveals

$$
L(B)-\epsilon \leqq L_{\delta}(B) \leqq \sum_{\Gamma \in \Theta}(\operatorname{diam} \widehat{\Gamma})=5 \sum_{\Gamma \in \Theta} \operatorname{diam} \Gamma \leqq \frac{5 \epsilon}{\eta-1}
$$

and we use the arbitrariness of $\epsilon$ to see that $L(B)=0$ in contradiction to (1).

The proof is complete.

5.17. Theorem. If $A \subset E_{2}$ and $L(A)<\infty$ then $\mathfrak{D}_{L}(A, x) \leqq 1$ for $L$ almost all $x$.

Proof. Relying on 3.4 let $B$ be a Borel set containing $A$ with $L(B)$ finite and note from $5.12,5.13$, and 5.16 that $\mathfrak{D}_{L}(B, x) \leqq 1$ for $L$ almost all $x$. Thus the desired conclusion is reached.

5.18. LEMMA. If $\phi \in \mathfrak{R}, A \subset E_{2}$, and $\mathfrak{D}_{\phi}^{\Delta}(x)>\mu \geqq 0$ for $L$ almost all $x$ in $A$, then $\mu L(A) \leqq 5 \phi\left(E_{2}\right)$.

Proof. Let $B=A E_{x}\left[\mathfrak{D}_{\phi}^{\Delta}(x)>\mu\right]$. Let $\delta$ be a positive number and $\mathfrak{F}$ be a family of circles covering $B$ such that

$$
(\operatorname{diam} \Gamma)<\delta / 5, \quad \phi(\Gamma) \geqq \mu \operatorname{diam} \Gamma \quad \text { for } \Gamma \in \mathfrak{F} .
$$

Relying on Theorem 5.11 , let $\&$ be a disjointed subfamily of $\mathfrak{F}$ such that 


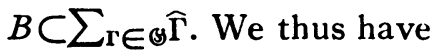

$$
\mu L_{\delta}(B) \leqq \mu \sum_{\Gamma \in \mathcal{S}} \operatorname{diam} \widehat{\Gamma}=5 \mu \sum_{\Gamma \in \mathcal{S}} \operatorname{diam} \Gamma \leqq 5 \sum_{\Gamma \in \mathcal{S}} \phi(\Gamma)=5 \phi[\sigma(\mathcal{S})] \leqq 5 \phi\left(E_{2}\right) .
$$

Therefore $\mu L(A)=\mu L(B) \leqq 5 \phi\left(E_{2}\right)$.

5.19. COROLLARY. If $\phi \in \mathfrak{M}$, then $E_{x}\left[\mathfrak{D}_{\phi}^{\Delta}(x)=\infty\right]$ has $L$ measure zero.

Using intersecting measure and applying Lemma 5.18 we have

5.20. LEMma. If $\phi \in \mathbb{M}, A \subset E_{2}$, and $\mathfrak{D}_{\phi}^{\Delta}(A, x)>\mu \geqq 0$ for $L$ almost all $x$ in $A$, then $\mu L(A) \leqq 5 \phi(A)$.

5.21. Lemma. If $\phi \in \mathfrak{M}, A$ is a Borel set, and $\mathfrak{D}_{\phi}^{\Delta}(x)>\mu \geqq 0$ for $L$ almost all $x$ in $A$, then $\mu L(A) \leqq 5 \phi(A)$.

Proof. Since $A$ is a Borel set we note from 5.14 that $\mathfrak{D}_{\phi}^{\Delta}(x)=\mathfrak{D}_{\phi}^{\Delta}(A, x)$ for $L$ almost all $x$ in $A$, and use Lemma 5.20.

5.22. Lemma. If $\phi \in \mathfrak{M}, A$ is a Borel set, and $\mathfrak{D}_{\phi}^{\Delta}(x)>0$ for $L$ almost all $x \in A$, then $L(B)=0$ whenever $B$ is a Borel subset of $A$ with $\phi(B)=0$.

Proof. Let $B$ be a Borel subset of $A$ with $\phi(B)=0$. Letting

$$
B_{n}=B \underset{x}{E}\left[\mathfrak{D}_{\phi}^{\Delta}(x)>1 / n\right]
$$

for each positive integer $n$, we see from Lemma 5.3 that $B_{n}$ is a Borel set and thus from Lemma 5.21 that $L\left(B_{n}\right)=0$. Furthermore $B_{1}+B_{2}+B_{3}+\cdots$ is $L$ almost all of $B$ and thus $L(B)=0$.

5.23. Lemma. If $\phi \in \mathfrak{M}, A \subset E_{2}$, and $\mathfrak{D}_{\phi}^{\Delta}(x)<\mu$ for $\phi$ almost all $x \in A$, then $\phi(A) \leqq 2 \mu L(A)$.

Proof. Let $\epsilon>0$. Relying on Lemma 5.8, determine a set $B C A$ and a number $\delta>0$ such that

$$
\phi(B) \geqq \phi(A)-\epsilon
$$

and

$$
\phi\left(\Omega_{x}^{r}\right) / 2 r \leqq \mu
$$

for $x \in B$ and $0<r<\delta$.

Also determine a family $\mathfrak{F}$ of open subsets of $E_{2}$ such that

$$
\begin{gathered}
B \subset \sigma(\mathfrak{F}), \quad L(B)+\epsilon \geqq \sum_{s \in \mathfrak{F}} \operatorname{diam} s ; \\
s B \neq 0, \quad \operatorname{diam} s<\delta / 2
\end{gathered}
$$

whenever $s \in \mathfrak{F}$.

For $s \in \mathfrak{F}$ let $\Gamma$, be a circle with center at a point of $s B$ and radius equal to 
$\operatorname{diam} s$. We then have the relation

$$
\begin{aligned}
\phi(A)-\epsilon & \leqq \phi(B) \leqq \sum_{s \in \mathfrak{F}} \phi(B s) \leqq \sum_{s \in \mathfrak{F}} \phi\left(\Gamma_{\diamond}\right) \leqq \mu \sum_{\mathfrak{s} \in \mathfrak{F}} \operatorname{diam} \Gamma_{\bullet} \\
& =2 \mu \sum_{s \in \mathfrak{F}} \operatorname{diam} s \leqq 2 \mu[L(B)+\epsilon] \leqq 2 \mu[L(A)+\epsilon] .
\end{aligned}
$$

The conclusion $\phi(A) \leqq 2 \mu L(A)$ follows.

5.24. Corollary. If $\phi \in \mathfrak{M}$ and $L(A)<\infty$, then $A E_{x}\left[\mathfrak{D}_{\phi}^{\Delta}(x)=0\right]$ has $\phi$ measure zero.

5.25. Lemma. If $\phi \in \mathfrak{M}$, and $\mathfrak{D}_{\phi}^{\Delta}(x)<\infty$ for $\phi$ almost all $x$, then corresponding to $\epsilon>0$ there is $a \delta>0$ such that $\phi(A)<\epsilon$ whenever $L(A)<\delta$.

Proof. Let $\epsilon>0$. Take $\mu>0$ so large that $\phi(B)<2^{-1} \epsilon$ where

$$
B=E\left[\mathfrak{D}_{\phi}^{\Delta}(x) \geqq \mu\right] \text {. }
$$

Putting $\delta=\epsilon(4 \mu)^{-1}$ we use 5.23 and see: $L(A)<\delta$ implies

$$
\begin{aligned}
\phi(A) & \leqq \phi(A B)+\phi(A \widetilde{B}) \leqq \phi(B)+\phi(A \tilde{B}) \leqq \epsilon / 2+2 \mu L(A \tilde{B}) \\
& \leqq \epsilon / 2+2 \mu L(A)<\mid \epsilon / 2+\epsilon / 2=\epsilon .
\end{aligned}
$$

5.26. Corollary. If $\phi \in \mathfrak{M}$, and $\mathfrak{D}_{\phi}^{\Delta}(x)<\infty$ for $\phi$ almost all $x$, then $\phi(A)=0$ whenever $L(A)=0$.

5.27. TheOREM. If $\phi \in \mathfrak{U}, \mu>0, A$ is rectifiable, and $\mathfrak{D}_{\phi}^{\nabla}(x)<\mu$ for $\phi$ almost all $x \in A$, then $\phi(A) \leqq 10 \mu L(A)$.

Proof. Let $f$ be a function on the open interval $E_{x}[0<t<1]$ to $E_{2}$ which satisfies a Lipschitz condition and whose range $R$ includes $A$.

We divide the remainder of the proof into three parts.

PART I. If $\alpha \subset A$ and diam $\alpha=0$ then $\phi(\alpha)=0$.

Proof (by contradiction). Suppose $\phi(\alpha)>0$.

The set $\alpha$ is nonvacuous and there is a point $p$ such that

$$
\mid \alpha=\{p\}, \quad \mathfrak{D}_{\phi}^{\nabla}(p)=\infty .
$$

Consequently

$$
\phi\left(A \underset{x}{E}\left[\mathfrak{D}_{\phi}^{\nabla}(x)=\infty\right]\right) \geqq \phi(\alpha)>0
$$

in contradiction to the hypothesis that $\mathfrak{D}_{\phi}^{\nabla}(x)<\mu$ for $\phi$ almost all $x$ in $A$.

PART II. If $\alpha \subset A$ and $\alpha \subset \beta \subset R$ with $\beta$ connected, then $\phi(\alpha) \leqq 10 \mu L(\beta)$.

Proof. If $\operatorname{diam} \beta=0$ then diam $\alpha=0$ and $\phi(\alpha)=0$ by part I.

We therefore assume $\operatorname{diam} \beta>0$.

Let $\alpha^{\prime}=\alpha E_{x}\left[\mathfrak{D}_{\phi}^{\nabla}(x)<\mu\right]$ and note that 


$$
\phi\left(\alpha^{\prime}\right)=\phi(\alpha) .
$$

Let $\mathfrak{F}$ be the family of circles such that $\Gamma \in \mathfrak{F}$ if and only if there is an $x \in \alpha^{\prime}$ and an $r$ with

$$
0<r \leqq \operatorname{diam} \beta / 2
$$

such that

$$
\Gamma=\Omega_{x}^{r} \text { and } \phi(\widehat{\Gamma})<\mu \operatorname{diam}(\widehat{\Gamma}) .
$$

Clearly $\mathfrak{F}$ covers $\alpha^{\prime}$. Furthermore, since $\beta$ is connected, we have from (2) and Theorem 4.2 that

$$
\Gamma \in \mathfrak{F} \text { implies }(\operatorname{diam} \Gamma) \leqq 2 L(\beta \Gamma) .
$$

Now select, according to Theorem 5.11 , a countable disjointed subfamily \& of $\mathfrak{F}$ such that

$$
\alpha^{\prime} \subset \sum_{\Gamma \in \Theta} \widehat{\Gamma}
$$

From (1), (5), (3), Abbreviation 2.25, (4) and the disjointedness of $\$ 3$ we have

$$
\begin{aligned}
\phi(\alpha) & =\phi\left(\alpha^{\prime}\right) \leqq \phi\left(\sum_{\Gamma \in \circlearrowleft} \widehat{\Gamma}\right) \leqq \sum_{\Gamma \in \Theta} \phi(\widehat{\Gamma}) \leqq \sum_{\Gamma \in \Theta} \mu(\operatorname{diam} \widehat{\Gamma}) \\
& =5 \mu \sum_{\Gamma \in \Theta} \operatorname{diam} \Gamma \leqq 10 \mu \sum_{\Gamma \in \Theta} L(\beta \Gamma) \\
& =10 \mu L[\beta \sigma(\$)] \leqq 10 \mu L(\beta) .
\end{aligned}
$$

PART III. $\phi(A) \leqq 10 \mu L(A)$.

Proof. Let $\epsilon>0$ and, by 3.4 , let $D$ be an open set containing $A$ such that

$$
L(D R) \leqq L(A)+\epsilon .
$$

Let $\mathfrak{F}$ be the disjointed family of open intervals such that $\sigma(\mathfrak{F})$ is the open set $E_{t}[f(t) \in D]$, and, for $I \in \mathfrak{F}$, let

$$
I^{*}=\sum_{t \in t}\{f(t)\}
$$

Now letting

$$
\overline{\mathfrak{F}}=\sum_{I \in \Im}\left\{I^{*}\right\}
$$

we note that $\overline{\mathfrak{F}}$ is a countable family of connected Borel subsets of $E_{2}$ with

$$
D R=\sum_{I \in \mathfrak{F}} \sum_{t \in I}\{f(t)\}=\sum_{I \in \mathfrak{F}} I^{*}=\sigma(\overline{\mathfrak{F}}) .
$$

We next select a finite subfamily $\mathbb{B}$ of $\bar{F}$ such that

$$
\phi(D R \tilde{C})<\epsilon
$$


where

$$
C=\sigma(\$)
$$

Notice that

$$
C \subset D R
$$

and

$$
\phi(A)=\phi(A C)+\phi(A \tilde{C})=\phi(A C)+\phi(D R \tilde{C})<\phi(A C)+\epsilon .
$$

Let $\mathfrak{S}$ be the family such that $\beta \in \mathfrak{W}$ if and only if $\beta$ is of the form $\sigma(\Im)$ where: $\Im$ is a subfamily of $\mathscr{S} ; \sigma(\Im)$ is connected; and $\sigma(\Im) \sigma(\Im)-\Im)=0$. We note that $\mathfrak{S}$ is a finite disjointed family of connected Borel sets.

If it were possible for $s$ to be a member of $\&$ and to be a subset of no member of $\mathfrak{E}$ we could be led by an iterative procedure to the false proposition that $\mathbb{S}$ is infinite. Accordingly $C \subset \sigma(\mathfrak{W})$ and, since it is evident that $\sigma(\mathfrak{W}) \subset C$, we conclude that

$$
C=\sigma(\mathfrak{S}) \text {. }
$$

Recalling that $\mathfrak{S}$ is a disjointed family of connected Borel sets we use (8), (9), Part II, (7), (6) to obtain

$$
\begin{aligned}
\phi(A) & \leqq \phi(A C)+\epsilon=\phi[A \sigma(\mathfrak{D})]+\epsilon \leqq \sum_{\beta \in \mathfrak{F}} \phi(A \beta)+\epsilon \\
& \leqq 10 \mu \sum_{\beta \in \mathfrak{F}} L(\beta)+\epsilon=10 \mu L[\sigma(\mathfrak{D})]+\epsilon \\
& =10 \mu L(C)+\epsilon \leqq 10 \mu L(D B)+\epsilon \leqq 10 \mu[L(A)+\epsilon]+\epsilon .
\end{aligned}
$$

Therefore $\phi(A) \leqq 10 \mu L(A)$.

The proof of 5.27 is complete.

5.28. Corollary. If $\phi \in \mathfrak{U}$, then $A E_{x}\left[\mathfrak{D}_{\phi}^{\nabla}(x)=0\right]$ has $\phi$ measure zero for each rectifiable set $A$.

5.29. Corollary. If $\phi \in \mathfrak{M}, \mathfrak{D}_{\phi}^{\nabla}(x)<\infty$ for $\phi$ almost all $x$, then $\phi(B)=0$ whenever $B$ is a rectifiable set for which $L(B)=0$.

5.30. TheOREM. If $\phi \in \mathfrak{M}, \mu>0, \phi(A)>0, \mathfrak{D}_{\phi}^{\Delta}(x)>\mu$ for $x \in A$, and if $\psi$ is the function on $\varepsilon_{2}$ such that $s \subset E_{2}$ implies $\psi(s)$ is the infimum of numbers of the form $\phi(S)$ where $S$ is an open set containing $s$, then $\psi \in \mathfrak{M}$ and there is a point $x_{0} \in A$ such that

$$
\mathfrak{D}_{\psi}^{\Delta}\left(A, x_{0}\right)>\mu / 7 \text {. }
$$

Proof. A simple check shows that $\psi \in \mathfrak{M}$. In order to prove there is a point $x_{0} \in A$ such that

$$
\mathfrak{D}_{\psi}^{\Delta}\left(A, x_{0}\right)>\mu / 7
$$


we shall show that the relation,

$$
\mathfrak{D}_{\psi}^{\Delta}(A, x) \leqq \mu / 7
$$

for $x \in A$,

leads to a contradiction.

Let $\epsilon>0$, let $H$ be an open set such that $H \subset A$ and

$$
\phi(H) \leqq \psi(A)+\epsilon,
$$

and let $\mathfrak{F}$ be the family of circles $\Gamma$ such that

$$
\Gamma \subset H, \quad 0<\operatorname{diam} \Gamma<1, \quad \psi(A \widehat{\Gamma})<5 \phi(\Gamma) / 6 .
$$

In the next paragraph we show that $\mathfrak{F}$ covers $A$.

Supposing $x \in A$, choose positive numbers $r_{1}, r_{2}, r_{3}, \cdots$ such that $r_{n} \rightarrow 0$ as $n \rightarrow \infty$ :

$$
0 \leqq \lim _{n \rightarrow \infty} 2 r_{n} / \phi\left(\Omega_{x}^{r_{n}}\right)<1 / \mu .
$$

With the aid of (1) we find

$$
\varlimsup_{n \rightarrow \infty} \frac{\psi\left(A \Re_{x}^{b r_{n}}\right)}{\phi\left(\Omega_{x}^{r_{n}}\right)}=5 \varlimsup_{n \rightarrow \infty}\left[\frac{\psi\left(A \Omega_{x}^{5 r_{n}}\right)}{10 r_{n}} \cdot \frac{2 r_{n}}{\phi\left(\Omega_{x}^{r_{n}}\right)}\right] \leqq 5 \mathfrak{D}_{\psi}^{\Delta}(A, x) \cdot \frac{1}{\mu} \leqq \frac{5}{7}<\frac{5}{6} .
$$

Accordingly for $n$ sufficiently large $\Omega_{x}^{r_{n}} \in \mathfrak{F}$ and $x \in \Omega_{z}^{r_{n}} \subset \sigma(\mathfrak{F})$.

Now use Theorem 5.11 to select a countable disjointed subfamily $\mathbb{B}$ of $\mathfrak{F}$ for which $A \subset \sum_{\mathrm{r} \in \Theta} \widehat{\Gamma}$. Therefore, remembering (2), we infer the relation

$$
\begin{aligned}
\psi(A)=\psi\left(\sum_{\Gamma \in \Theta} A \widehat{\Gamma}\right) & \leqq \sum_{\mathrm{r} \in \circlearrowleft} \psi(A \widehat{\Gamma})<\frac{5}{6} \sum_{\mathrm{r} \in \mathcal{S}} \phi(\Gamma)=\frac{5}{6} \phi[\sigma(\circlearrowleft)] \\
& \leqq \frac{5}{6} \phi(H) \leqq \frac{5}{6}[\psi(A)+\epsilon]
\end{aligned}
$$

from which follows the false proposition

$$
0<\phi(A) \leqq \psi(A) \leqq \frac{5}{6} \psi(A)<\psi(A) .
$$

5.31. Lemma. If $\phi \in \mathbb{M}, A \subset E_{2}$,

$$
\mathfrak{D}_{\phi}^{\Delta}(x)>0 \text { and } \mathfrak{D}_{\phi}^{\Delta}(A, x)=0 \text { for } x \in A,
$$

then $\phi(A)=0$.

Proof. Let $\epsilon>0, \eta=\epsilon\left[\phi\left(E_{2}\right)+1\right]^{-1}$ and $\mathfrak{F}$ be the family of circles $\Gamma$ such that $0<\operatorname{diam} \Gamma<1$ and $\phi(A \widehat{\Gamma})<\eta \phi(\Gamma)$. In the next paragraph we show that $\mathfrak{F}$ covers $A$.

Letting $x \in A$, choose positive numbers $r_{1}, r_{2}, r_{3}, \cdots$, such that: $r_{n} \rightarrow 0$ as $n \rightarrow \infty$; 


$$
0 \leqq \lim _{n \rightarrow \infty} \frac{2 r_{n}}{\phi\left(\AA_{x}^{r_{n}}\right)}<\infty
$$

We have

$$
\lim _{n \rightarrow \infty} \frac{\phi\left(A \Omega_{x}^{5 r_{n}}\right)}{\phi\left(\Omega_{x}^{r_{n}}\right)}=5 \lim _{n \rightarrow \infty}\left[\frac{\phi\left(A \Re_{x}^{b r_{n}}\right)}{10 r_{n}} \cdot \frac{2 r_{n}}{\phi\left(\Omega_{x}^{r_{n}}\right)}\right]=0 .
$$

Accordingly for $n$ sufficiently large $\Omega_{x}^{r_{n}} \in \mathfrak{F}$ and $x \in \Omega_{x}^{r_{n}} \subset \sigma(\mathfrak{F})$.

Now use Theorem 5.11 to select a countable disjointed subfamily $\mathbb{B}$ of $\mathfrak{F}$ for which $A \subset \sum_{\mathrm{r} \in \mathbb{\Gamma}} \widehat{\Gamma}$, and note that

$$
\phi(A)=\phi\left(\sum_{\Gamma \in \Theta} \dot{A} \widehat{\Gamma}\right) \leqq \sum_{\Gamma \in \Theta} \phi(A \widehat{\Gamma}) \leqq \eta \sum_{\Gamma \in \Theta} \phi(\Gamma)=\eta \phi[\sigma(\Im)] \leqq \eta \phi\left(E_{2}\right)+\epsilon .
$$

Thus $\phi(A)=0$.

5.32. THEOREM. If $\phi \in \mathfrak{M}, A \subset E_{2}$, and $\mathfrak{D}_{\phi}^{\Delta}(x)>0$ for $\phi$ almost all $x \in A$, then $\mathfrak{D}_{\phi}^{\Delta}(A, x)>0$ for $\phi$ almost all $x \in A$.

Proof. Let

$$
C=A \underset{x}{E}\left[\mathfrak{D}_{\phi}^{\Delta}(x)>0\right] \quad \text { and } \quad B=A \underset{x}{E}\left[\mathfrak{D}_{\phi}^{\Delta}(A, x)=0\right] \text {. }
$$

Consequently $\mathfrak{D}_{\phi}^{\Delta}(B C, x)=0$ for $x \in B C$, and we use Lemma 5.31 to obtain

$$
0=\phi(B C)=\phi(B) \text {. }
$$

5.33. TheOREM. If $\phi \in \mathfrak{U}, A \subset B, \phi(B)<\infty$, and $\mathfrak{D}_{\phi}^{\Delta}(B, x)>0$ for $\phi$ almost all $x \in A$, then $\mathfrak{D}_{\phi}^{\Delta}(A, x)>0$ for $\phi$ almost all $x$ in $A$.

Proof. Use intersecting measure on $B$.

6. Rectifiability and directionality. Theorems $6.1,6.2$, and 6.3 which follow are rather immediate consequences of 2.17 .

6.1. Theorem. Sgn $(A, x)=0$ if and only if $A \subset\{x\} \subset E_{2}$.

6.2. Theorem. If $x \in E_{2}, A \subset E_{2}$, then

$$
\operatorname{dir}(A, x)=\prod_{r \in \Re} \operatorname{Sgn}\left(A K_{r}, x\right)
$$

where $\Re$ is any set of positive numbers with 0 as a cluster point.

6.3. ThEOREM. If $x \in E_{2}, A \subset E_{2}$, then

$$
\operatorname{dir}_{\phi}(A, x)=\prod_{\beta \in \mathfrak{S}} \operatorname{Sgn}(A \tilde{\beta}, x)
$$

where

$$
\mathfrak{S}=\underset{\beta}{E}\left[\beta \subset A, \mathfrak{D}_{\phi}^{\Delta}(\beta, x)=0\right]
$$


Recalling 2.8 we obtain

6.4. TheOREM. If $\phi$ is counting measure, $x \in E_{2}$, and $A \subset E_{2}$, then

$$
\operatorname{dir}_{\phi}(A, x)=\operatorname{dir}(A, x) .
$$

6.5. Theorem. If $\phi \in \mathfrak{u}, A \subset E_{2}, B \subset E_{2}$, and $x \in E_{2}$, then

(i) $\operatorname{Sgn}(A+B, x)=\operatorname{Sgn}(A, x)+\operatorname{Sgn}(B, x)$,

(ii) $\operatorname{dir}(A+B, x)=\operatorname{dir}(A, x)+\operatorname{dir}(B, x)$,

(iii) $\operatorname{dir}_{\phi}(A+B, x)=\operatorname{dir}_{\phi}(A, x)+\operatorname{dir}_{\phi}(B, x)$.

Proof. Since the closure of the union of two sets is the union of the closures of these sets we see that (i) holds. If $\beta_{1}$ and $\beta_{2}$ are sets such that $\mathfrak{D}_{\phi}^{\Delta}\left(\beta_{1}, x\right)$ $=\mathfrak{D}_{\phi}^{\Delta}\left(\beta_{2}, x\right)=0$, then $\mathfrak{D}_{\phi}^{\Delta}\left(\beta_{1}+\beta_{2}, x\right)=0$; and we see that (iii) holds. Letting $\phi$ be the counting measure of 2.8 and 3.14, we see that (ii) follows from (iii).

6.6. Theorem. If $\phi \in \mathfrak{U}, x \in A \subset E_{2}$, and $C$ is a bounded closed set with $C \operatorname{dir}_{\phi}(A, x)=0$, then there is a set $B C A$ such that $\mathfrak{D}_{\phi}^{\Delta}(B, x)=0$ and $C \operatorname{Sgn}(A \widetilde{B}, x)=0$.

Proof. For $z \in C$ : let $\beta_{z}$ be such that

$$
\beta_{z} \subset A, \mathfrak{D}_{\phi}^{\Delta}\left(\beta_{z}, x\right)=0 ;\{z\} \operatorname{Sgn}\left(A \tilde{\beta}_{z}, x\right)=0 ;
$$

recall that $\operatorname{Sgn}\left(A \tilde{\beta}_{z}, x\right)$ is closed; take $S_{z}$ to be an open set of which $z$ is an element and for which $S_{z} \operatorname{Sgn}\left(A \widetilde{\beta}_{z}, x\right)=0$. The Heine-Borel Theorem allows us to select a finite subset $C^{\prime}$ of $C$ such that

$$
C \subset \sum_{z \in C^{\prime}} S_{z}
$$

Let ting

$$
B=\sum_{z \in C^{\prime}} \beta_{z}
$$

checking that

$$
\mathfrak{D}_{\phi}^{\Delta}(B, x) \leqq \sum_{z \in C^{\prime}} \mathfrak{D}_{\phi}^{\Delta}\left(\beta_{z}, x\right)=0,
$$

we observe that $z \in C^{\prime}$ implies

$$
S_{z} \operatorname{Sgn}(A \tilde{B}, x) \subset S_{z} \operatorname{Sgn}\left(A \tilde{\beta}_{z}, x\right)=0 .
$$

Thus

$$
C \operatorname{Sgn}(A \tilde{B}, x) \subset \sum_{z \in C^{\prime}} S_{z} \operatorname{Sgn}(A \widetilde{B}, x)=0
$$

and the proof is complete.

By taking $C=\mathbb{C}_{(0,0)}^{1}$ in the above theorem we have the 
6.7. Theorem. If $\phi \in \mathfrak{U}$ and $A \subset E_{2}$ then $\operatorname{dir}_{\phi}(A, x)=0$ if and only if $\mathfrak{D}_{\phi}^{\Delta}(A-\{x\}, x)=0$.

Letting $\phi$ be counting measure in Theorem 6.7 we have

6.8. Corollary. If $A \subset E_{2}$ then $\operatorname{dir}(A, x) \neq 0$ if and only if $x$ is a cluster point of $A$.

Using 6.7 and Theorem 5.12 we have the following

6.9. Corollary. If $\phi \in \mathfrak{M}, A$ is a Borel set, then $\operatorname{dir}_{\phi}(\tilde{A}, x)=0$ for $L$ almost all $x \in A$.

From 6.5(iii) and Corollary 6.9 we have the

6.10. Theorem. If $\phi \in \mathfrak{M}$ and $A$ is a Borel subset of $E_{2}$, then $\operatorname{dir}_{\phi}(A, x)$ $=\operatorname{dir}_{\phi}(x)$ for $L$ almost all $x \in A$.

Use of intersecting measure leads to the

6.11. Corollary. If $\phi \in \mathfrak{U}, B \subset A \subset E_{2}, \phi(A)<\infty$, and $B$ is a Borel set, then $\operatorname{dir}_{\phi}(B, x)=\operatorname{dir}_{\phi}(A, x)$ for $L$ almost all $x \in B$.

6.12. TheOREM. If $A$ is strictly rectifiable, then there is a set $W \subset A$ with $L(A \tilde{W})=0$ such that $x \in W$ implies:

(i) $A$ is directional at $x$;

(ii) $\mathfrak{D}_{L}^{\nabla}(A, x)=\mathfrak{D}_{L}^{\wedge}(A, x)=1$;

(iii) corresponding to $\xi \in \operatorname{dir}(A, x)$ there is a continuum $\alpha \subset A$ such that $\mathfrak{D}_{L}^{\nabla}(\alpha, x)=1 / 2$ and $\operatorname{dir}(\alpha, x)=\xi$.

Proof. If diam $A=0$ then $L(A)=0$ and the theorem is trivial.

We assume henceforth that diam $A>0$.

Let $g$ be a function on $[0,1]$ to $E_{2}$ which satisfies a Lipschitz condition and whose range is $A$. Using the process known as parametrizing according to arc length, we ascertain a number $l>0$ and a function $f$ on $[0, l]$ with range $A$ for which

$$
\begin{aligned}
& \left|f\left(t^{\prime \prime}\right)-f\left(t^{\prime}\right)\right|<t^{\prime \prime}-t^{\prime} \text { for } 0 \leqq t^{\prime} \leqq t^{\prime \prime} \leqq l, \\
& \left|f^{\prime}(t)\right|=1 \text { for Lebesgue almost all } t \text { in }[0, l] .
\end{aligned}
$$

We now define

$$
\begin{aligned}
& T_{1}=[0, l] \underset{t}{E}\left[\left|f^{\prime}(t)\right| \neq 1\right]+\{0\}+\{l\}, \\
& B_{1}=\sum_{t \in T_{1}}\{f(t)\}, \\
& B_{2}=A \underset{x}{E}\left[\mathfrak{D}_{L}(A, x)>1\right], \\
& W=A \widetilde{B}_{1} \widetilde{B}_{2} .
\end{aligned}
$$


Thus $L\left(B_{2}\right)=0$ by Theorem 5.17. From (1) we conclude $L\left(B_{1}\right) \leqq($ Lebesgue measure of $\left.T_{1}\right)=0$. Therefore

$$
L(A \tilde{W})=0 .
$$

We set about showing that $W$ has the additional properties ascribed to it by the theorem. To this end let $x$ be any point in $W$ and select a number $\tau$, with $0<\tau<l$, for which $x=f(\tau)$. Define $z=f^{\prime}(\tau)$, note that $|z|=1$, take $\delta>0$ such that

$$
0<|t-\tau|<\delta \text { implies }\left|\frac{f(t)-f(\tau)}{t-\tau}-z\right|<1 / 2
$$

Let

$$
A_{1}=\sum_{t \in[\tau-\delta, \tau]}\{f(t)\}, \quad A_{2}=\sum_{t \in[\tau, \tau+\delta]}\{f(t)\}, \quad A_{3}=A_{1}+A_{2} .
$$

Note that $A_{1}, A_{2}$ and $A_{3}$ are closed connected sets (continua).

We divide the remainder of the proof into 6 parts.

PART I. If $t^{\prime}<t^{\prime \prime}, \tau-\delta \leqq t^{\prime} \leqq \tau \leqq t^{\prime \prime} \leqq \tau+\delta$, then $0<\left|f\left(t^{\prime \prime}\right)-f\left(t^{\prime}\right)\right|$.

Proof (by contradiction). Assuming $f\left(t^{\prime}\right)=f\left(t^{\prime \prime}\right)$ we infer from (2) that

$$
\begin{aligned}
0<t^{\prime \prime}-t^{\prime} & =|z|\left(t^{\prime \prime}-t^{\prime}\right)=\left|\left(t^{\prime \prime}-\tau\right) z+\left(\tau-t^{\prime}\right) z\right| \\
& \leqq\left|\left[t^{\prime \prime}-\tau\right] z-\left[f\left(t^{\prime \prime}\right)-f(\tau)\right]\right|+\left|\left[\tau-t^{\prime}\right] z-\left[f(\tau)-f\left(t^{\prime}\right)\right]\right| \\
& \leqq\left(t^{\prime \prime}-\tau\right) / 2+\left(\tau-t^{\prime}\right) / 2=\left(t^{\prime \prime}-t^{\prime}\right) / 2 .
\end{aligned}
$$

As an immediate consequence of Part I we have

Part II. $A_{1} A_{2}=\{x\}$, diam $A_{1}>0, \operatorname{diam} A_{2}>0$.

PART III. $\mathfrak{D}_{L}^{\nabla}\left(A_{1}, x\right)=1 / 2, \mathfrak{D}_{L}^{\nabla}\left(A_{2}, x\right)=1 / 2$,

$$
\mathfrak{D}_{L}^{\nabla}(A, x)=\mathfrak{D}_{L}^{\Delta}(A, x)=\mathfrak{D}_{L}^{\nabla}\left(A_{3}, x\right)=\mathfrak{D}_{L}^{\Delta}\left(A_{3}, x\right)=1, \quad \mathfrak{D}_{L}^{\Delta}\left(A \tilde{A}_{3}\right)=0 .
$$

Proof Since $A_{1}$ and $A_{2}$ are continua with positive diameter, it follows from Theorem 4.2 that $\mathfrak{D}_{L}^{\nabla}\left(A_{1}, x\right) \geqq 1 / 2$ and $\mathfrak{D}_{L}^{\nabla}\left(A_{2}, x\right) \geqq 1 / 2$. Hence, since $A_{1}+A_{2}=A_{3}, L\left(A_{1} A_{2}\right)=L(\{x\})=0$ and $\mathfrak{D}_{L}^{\wedge}(A, x) \leqq 1$, we infer

$$
\begin{aligned}
1 & \leqq \mathfrak{D}_{L}^{\nabla}\left(A_{1}, x\right)+\mathfrak{D}_{L}^{\nabla}\left(A_{2}, x\right) \leqq \mathfrak{D}_{L}^{\nabla}\left(A_{1}+A_{2}, x\right)=\mathfrak{D}_{L}^{\nabla}\left(A_{3}, x\right) \\
& \leqq \mathfrak{D}_{L}^{\nabla}(A, x) \leqq \mathfrak{D}_{L}^{\wedge}(A, x) \leqq 1 .
\end{aligned}
$$

Furthermore, inasmuch as $\mathfrak{D}_{L}^{\nabla}\left(A_{3}, x\right) \leqq \mathfrak{D}_{L}^{A}\left(A_{3}, x\right) \leqq \mathfrak{D}_{L}^{\Lambda}(A, x)$, we also have $\mathfrak{D}_{L}\left(A_{3}, x\right)=1$. Using that $A_{3}$ is an $L$ measurable (closed) subset of $A$, we obtain

$$
0 \leqq \mathfrak{D}_{L}^{\Delta}\left(A \tilde{A}_{3}, x\right) \leqq \mathfrak{D}_{L}^{\Delta}(A, x)-\mathfrak{D}_{L}^{\nabla}\left(A_{3}, x\right)=1-1=0
$$

and thus Part III is proved.

PART IV. dir $\left(A_{1}, x\right)=\{-z\}, \operatorname{dir}\left(A_{2}, x\right)=\{z\}$. 
Proof. Let $q_{1}, q_{2}, q_{3}, \ldots$ be points distinct from $x$ with $q_{n} \rightarrow x$ as $n \rightarrow \infty$ and such that either

$$
q_{n} \in A_{1} \text { for } n=1,2,3, \cdots \quad \text { or } \quad q_{n} \in A_{2} \text { for } n=1,23, \cdots
$$

For each positive integer $n$ : let $t_{n}$ be such that $f\left(t_{n}\right)=q_{n}$ and $\left|t_{n}-\tau\right|<\delta$; note that (2) helps us infer

$$
\left|q_{n}-x\right|=\left|f\left(t_{n}\right)-f(\tau)\right| \geqq\left|t_{n}-\tau\right| / 2 .
$$

Accordingly $t_{n} \rightarrow \tau$ as $n \rightarrow \infty$. By letting $\nu=-1$ or +1 according as $q_{1}$ is or is not an element of $A_{1}$, we see that $\nu\left(t_{n}-\tau\right)>0$ for each positive integer $n$, and also see that

$$
\begin{aligned}
\lim _{n \rightarrow \infty} \frac{q_{n}-x}{\left|q_{n}-x\right|} & =\lim _{n \rightarrow \infty} \frac{f\left(t_{n}\right)-f(\tau)}{\left|f\left(t_{n}\right)-f(\tau)\right|}=\lim _{n \rightarrow \infty} \frac{f\left(t_{n}\right)-f(\tau)}{\nu\left(t_{n}-\tau\right)} \cdot \frac{\nu\left(t_{n}-\tau\right)}{\left|f\left(t_{n}\right)-f(\tau)\right|} \\
& =\nu z \lim _{n \rightarrow \infty}\left|\frac{t_{n}-\tau}{f\left(t_{n}\right)-f(\tau)}\right|=\nu \frac{z}{|z|}=\nu z .
\end{aligned}
$$

PAkT V. $\operatorname{dir}(A, x)=\{-z\}+\{z\}$ and $A$ is directional at $x$.

Proof. Let $\xi$ be any point in $\operatorname{dir}(A, x)$. Let $p_{1}, p_{2}, p_{3}, \cdots$ be points in $A$ distinct from $x$ with

$$
\lim _{n \rightarrow \infty} p_{n}=x, \quad \lim _{n \rightarrow \infty} \frac{p_{n}-x}{\left|p_{n}-x\right|}=\xi .
$$

For each positive integer $n$ : let

$$
\rho_{n}=\operatorname{dist}\left(\left\{p_{n}\right\}, A_{3}\right) / 2, \quad r_{n}=\left|p_{n}-x\right|+\rho_{n} ;
$$

check that

$$
\begin{gathered}
\rho_{n} \leqq 2 \rho_{n} \leqq\left|p_{n}-x\right|, \quad \Omega_{p_{n}}^{\rho_{n}} \subset \Omega_{x}^{r_{n}}, \quad A{ }_{3} \Re_{p_{n}}^{\rho_{n}}=0, \\
A \tilde{A}_{3} \Omega_{x}^{r_{n}} \supset A \tilde{A}_{3} \Omega_{x}^{r_{n}} \Omega_{p_{n}}^{\rho_{n}}=A \tilde{A}_{3} \Omega_{p_{n}}^{\rho_{n}}=A \Omega_{p_{n}}^{\rho_{n}} ;
\end{gathered}
$$

observe that Theorem 4.2 implies

$$
L\left(A \Re_{p_{n}}^{\rho_{n}}\right) \geqq \rho_{n} ;
$$

infer

$$
\begin{aligned}
0<\left|p_{n}-x\right| & \leqq r_{n} \leqq 2\left|p_{n}-x\right| ; \\
\frac{L\left(A \tilde{A}_{3} \Omega_{x}^{r_{n}}\right)}{2 r_{n}} & \geqq \frac{L\left(A \Omega_{p n}^{r_{n}}\right)}{2 r_{n}} \geqq \frac{\rho_{n}}{4\left|p_{n}-x\right|} .
\end{aligned}
$$

Thus inasmuch as $\mathfrak{D}_{\hat{L}}\left(A \tilde{A}_{3}, x\right)=0$ by Part III, we see from (5) and (6) that

$$
\lim _{n \rightarrow \infty} \frac{\rho_{n}}{\left|p_{n}-x\right|}=0
$$


Now, for each positive integer $n$ : recall the definition (4) of $\rho_{n}$; remember that $A_{3}$ is closed; choose $q_{n}$ so that

$$
q_{n} \in A_{3}, \quad\left|p_{n}-q_{n}\right|=2 \rho_{n} \leqq\left|p_{n}-x\right| ;
$$

and note

$$
\left|q_{n}-x\right| \geqq\left|p_{n}-x\right|-\left|p_{n}-q_{n}\right|=\left|p_{n}-x\right|-2 \rho_{n} .
$$

With (7) in mind select a positive number $N$ so that $n>N$ implies $2 \rho_{n}<\left|p_{n}-x\right|$ which in turn, by virtue of (9), implies $\left|q_{n}-x\right|>0$. Consequently $n>N$ implies

$$
\begin{aligned}
\left|\frac{p_{n}-x}{\left|p_{n}-x\right|}-\frac{q_{n}-x}{\left|q_{n}-x\right|}\right| & =\left|\frac{p_{n}-q_{n}+q_{n}-x}{\left|p_{n}-x\right|}-\frac{q_{n}-x}{\left|q_{n}-x\right|}\right| \\
& \leqq \frac{2 \rho_{n}}{\left|p_{n}-x\right|}+\left|\frac{q_{n}-x}{\left|p_{n}-x\right|}-\frac{q_{n}-x}{\left|q_{n}-x\right|}\right| \\
& =\frac{2 \rho_{n}}{\left|p_{n}-x\right|}+\left|q_{n}-x\right|\left|\frac{\left|q_{n}-x\right|-\left|p_{n}-x\right|}{\left|p_{n}-x\right|\left|q_{n}-x\right|}\right| \\
& \leqq \frac{2 \rho_{n}}{\left|p_{n}-x\right|}+\frac{\left|q_{n}-p_{n}\right|}{\left|p_{n}-x\right|}=\frac{4 \rho_{n}}{\left|p_{n}-x\right|} .
\end{aligned}
$$

This last relation, (7), and (3) combine to yield

$$
\lim _{n \rightarrow \infty} \frac{q_{n}-x}{\left|q_{n}-x\right|}=\lim _{n \rightarrow \infty} \frac{p_{n}-x}{\left|p_{n}-x\right|}=\xi
$$

and, since (3) and (8) assure us that $q_{n} \rightarrow x$ with $q_{n}$ in $A_{8}$ as $n \rightarrow \infty$, it is immediate that

$$
\xi \in \operatorname{dir}\left(A_{3}, x\right) .
$$

By our choice of $\xi$ we have now shown

$$
\operatorname{dir}(A, x) \subset \operatorname{dir}\left(A_{3}, x\right) \subset \operatorname{dir}(A, x),
$$

so that use of 6.5 (iii) and Part IV yields

$$
\operatorname{dir}(A, x)=\operatorname{dir}\left(A_{3}, x\right)=\operatorname{dir}\left(A_{1}, x\right)+\operatorname{dir}\left(A_{2}, x\right)=\{-z\}+\{z\} .
$$

Referring to 2.19 and 2.20 we conclude at once that $A$ is directional at $x$.

PART VI. If $\xi \in \operatorname{dir}(A, x)$, then there is a continuum $\alpha \subset A$ such that $\mathfrak{D}_{L}^{\nabla}(\alpha, x)=1 / 2$ and $\operatorname{dir}(\alpha, x)=\xi$.

Proof. From Part V we know $\xi=-z$ or $\xi=z$. Recalling Part III we take $\alpha=A_{1}$ in the event $\xi=-z$ and take $\alpha=A_{2}$ in the event $\xi=z$. Since the sets $A_{1}$ and $A_{2}$ are continua the proof is complete.

6.13. LEMMA. If $\phi \in \mathfrak{M}, A$ is strictly rectifiable, $\mu>0, \mathfrak{D}_{\phi}^{\Delta}(x)>\mu$ for $L$ al- 
most all $x$ in $A$, and $\mathfrak{D}_{\phi}^{\nabla}(x)<\infty$ for $\phi$ almost all $x$, then $A$ is $\phi$ directional at $\phi$ almost all of its points.

Proof. Identifying the notation of the theorem and that of 6.12 we let $W$ be the set whose existence is assured by 6.12 , and let $y$ be any point in $W$. Since $L(A \tilde{W})=0$, Remark 2.15 (ii) and Corollary 5.29 enables us to infer $\phi(A \tilde{W})=0$. Therefore, since $A$ is directional at $y$ according to 6.12 , we know that $\operatorname{dir}(A, x)$ is diametral and that completion of our proof is tantamount to the verification of

$$
\operatorname{dir}_{\phi}(A, y)=\operatorname{dir}(A, y) .
$$

Select $\xi \in \operatorname{dir}(A, x)$ and let, as in $6.12, \alpha$ be a continuum contained in $A$ for which

$$
\mathfrak{D}_{L}^{\nabla}(\alpha, y) \geqq 1 / 2, \quad \operatorname{dir}(\alpha, y)=\{\xi\} .
$$

Since $\mathfrak{D}_{\phi}^{\Delta}(x)>\mu$ for $L$ almost all $x$ in $A$, we are assured by 5.21 of the following relation,

$$
\frac{\phi\left(\alpha \Omega_{y}^{r}\right)}{2 r} \geqq \frac{\mu}{5} \frac{L\left(\alpha \Omega_{y}^{r}\right)}{2 r}
$$

which convinces us that

$$
\mathfrak{D}_{\phi}^{\nabla}(\alpha, y) \geqq \mu / 10>0 .
$$

Thus, since $\phi(\{y\})=0$ by 5.29 , we have

$$
\mathfrak{D}_{\phi}^{\Delta}(\alpha-\{y\}, y)=\mathfrak{D}_{\phi}^{\Delta}(\alpha, y) \geqq \mathfrak{D}_{\phi}^{\nabla}(\alpha, y)>0 .
$$

Hence from 6.7 it follows that

$$
0 \neq \operatorname{dir}_{\phi}(\alpha, y) \subset \operatorname{dir}(\alpha, y)=\{\xi\} .
$$

Accordingly

$$
\xi \in \operatorname{dir}_{\phi}(\alpha, y) \subset \operatorname{dir}_{\phi}(A, y),
$$

and recalling the choice of $\xi$, it is apparent that

$$
\operatorname{dir}(A, y) \subset \operatorname{dir}_{\phi}(A, y) \subset \operatorname{dir}(A, y) .
$$

The validity of (1) is established and the proof is complete.

We later obtain as an incidental result that a strictly rectifiable set $A$ is $L$ directional at $L$ almost all points of $A$. This special result may now be ob tained by defining the function $\phi$ on $\varepsilon_{2}$ so that $\phi(S)=L(A S)$ for $S \subset E_{2}$ and applying Lemma 6.13 .

6.14. LEMMA. If $\phi \in \mathfrak{M}, A$ is a strictly rectifiable set, and $\mathfrak{D}_{\phi}^{\nabla}(x)<\infty$ for $\phi$ almost all $x$, then $\operatorname{dir}_{\phi}(x)$ is diametral for $\phi$ almost all $x$ in $A$. 
Proof. Let $\epsilon>0$. Since $A E_{x}\left[\mathfrak{D}_{\phi}^{\Delta}(x)=0\right]$ has $\phi$ measure zero by 5.24 , we select such a number $\mu$ that

$$
0<\mu<1, \quad \phi(A \tilde{B})<\epsilon,
$$

where $B$ is the Borel set $A E_{x}\left[\mathfrak{D}_{\phi}^{\Delta}(x)>\mu\right]$.

Let $\psi$ be the auxiliary function on $\varepsilon_{2}$ such that

$$
\psi(s)=\phi(B s)+L(A \tilde{B} s)
$$

Check that $\psi \in \mathbb{M}$.

From 2.15(ii) and 5.29 it is clear that $\phi(B s)=0$ whenever $L(s)=0$. Thus

$$
L(s)=0 \text { implies } \psi(s)=0 .
$$

Since Corollary 5.19 implies the set

$$
\underset{x}{E}\left[\mathfrak{D}_{\psi}^{\Delta}(x)=\infty\right]
$$

has $L$ measure zero, it follows from (2) that

$$
\mathfrak{D}_{\psi}^{\nabla}(x) \leqq \mathfrak{D}_{\psi}^{\Delta}(x)<\infty \quad \text { for } \psi \text { almost all } x .
$$

Since

$$
\psi(\beta)=\phi(\beta)
$$

we use 5.14 to infer

$$
\mathfrak{D}_{\psi}^{\Delta}(x)=\mathfrak{D}_{\psi}^{\Delta}(B, x)=\mathfrak{D}_{\phi}^{\Delta}(B, x)=\mathfrak{D}_{\phi}^{\Delta}(x)>\mu
$$

for $L$ almost all $x$ in $B$; on the other hand; using 5.15 and 6.12 , we also infer

$$
\mathfrak{D}_{\psi}^{\Delta}(x)=\mathfrak{D}_{\psi}^{\Delta}(\tilde{B}, x)=\mathfrak{D}_{L}^{\Delta}(A \tilde{B}, x)=\mathfrak{D}_{L}^{\Delta}(A, x)=1>\mu
$$

for $L$ almost all $x \in A \tilde{B}$. Relations (5) and (6) assure us that

$$
\mathfrak{D}_{\psi}^{\Delta}(x)>\mu \quad \text { for } L \text { almost all } x \text { in } A .
$$

We combine (3), (7) and 6.13 and discover that $A$ is $\psi$ directional at $\psi$ almost all of its points. Accordingly

$$
\operatorname{dir}_{\psi}(A, x) \text { is diametral }
$$

for $\psi$ almost all $x$ in $A$.

From (4) and 6.3 it may be inferred that

$$
\operatorname{dir}_{\psi}(B, x)=\operatorname{dir}_{\phi}(B, x) \quad \text { for } x \in B .
$$

From this and 6.11 it follows that

$$
\operatorname{dir}_{\psi}(A, x)=\operatorname{dir}_{\psi}(B, x)=\operatorname{dir}_{\phi}(B, x)=\operatorname{dir}_{\phi}(x)
$$


for $L$, and hence $\psi$, almost all $x$ in $B$. Consequently, in view of (8) and (9), we see that $\operatorname{dir}_{\phi}(x)$ is diametral for $\psi$ almost all $x$ in $B$.

Hence $\operatorname{dir}_{\phi}(x)$ is diametral for $\phi$ almost all $x$ in $B$.

Reference to (1) completes the proof.

6.15. TheOREM. If $\phi \in \mathfrak{U}, A$ is $\phi$ rectifiable, $\phi(A)<\infty$, and $\mathfrak{D}_{\phi}^{\nabla}(A, x)<\infty$ for $\phi$ almost all $x$ in $A$, then $A$ is $\phi$ directional at $\phi$ almost all of its points.

Proof (by contradiction). Choose $B$ so that $B C A, \phi(B)>0$, and $\operatorname{dir}_{\phi}(A, x)$ is nondiametral for $x \in B$. Use the $\phi$ rectifiability of $A$ to obtain a strictly rectifiable set $C$ for which $\phi(B C)>0$.

Let $\Phi$ be the function on $\varepsilon_{2}$ such that $\Phi(s)=\phi(A s)$ for $s \subset E_{2}$ and note: $\Phi \in \mathfrak{M} ; \mathfrak{D}_{\Phi}^{\nabla}(x)<\infty$ for $\Phi$ almost all $x$;

$$
\Phi(B C)=\phi(B C)>0 .
$$

Since $\Phi(\beta)=\phi(\beta)$ for each $\beta C A$ it follows from 6.5(iii) and 6.3 that $x \in E_{2}$ implies

$$
\operatorname{dir}_{\Phi}(x)=\operatorname{dir}_{\Phi}(A, x)+\operatorname{dir}_{\Phi}(\tilde{A}, x)=\operatorname{dir}_{\Phi}(A, x)=\operatorname{dir}_{\phi}(A, x) .
$$

Accordingly $\operatorname{dir}_{\Phi}(x)$ is nondiametral for each $x \in B C$, and 6.14 assures us that $\Phi(B C)=0$ in contradiction to (1).

6.16. Remark. In Theorem 6.15 the hypothesis that $\phi(A)<\infty$ may be dispensed with. We neither prove nor use this fact.

6.17 Lemмa. If $\phi \in \mathfrak{M}, \mathfrak{D}_{\phi}^{\nabla}(x)<\infty$ for $\phi$ almost all $x$, and $A$ is rectifiable, then there is a set $B C A$ such that: $\phi(A \widetilde{B})=0 ; B$ is directional at each of its points; and

$$
\operatorname{dir}(B, x)=\operatorname{dir}_{\phi}(B, x)=\operatorname{dir}_{\phi}(A, x)=\operatorname{dir}(A, x)
$$

for each $x \in B$.

Proof. Let $C$ be a strictly rectifiable set containing $A$ and define

$$
\begin{aligned}
W_{1} & =\underset{x}{C}[C \text { is directional at } x], \\
W_{2} & =\underset{x}{A}[A \text { is } \phi \text { directional at } x], \\
B & =W_{1} W_{2} .
\end{aligned}
$$

Note that $B C A$ and use $6.12,5.29$, and 6.15 to infer

$$
L\left(A \tilde{W}_{1}\right)=0=\phi\left(A \tilde{W}_{1}\right), \quad \phi\left(A \tilde{W}_{2}\right)=0, \quad \phi(A \tilde{B})=0 .
$$

Thus, with the aid of 6.5 (iii) and 6.7, verify that $x \in E_{2}$ implies

$$
\begin{aligned}
\operatorname{dir}_{\phi}(A, x) & =\operatorname{dir}_{\phi}(A \tilde{B}, x)+\operatorname{dir}_{\phi}(B, x) \\
& =\operatorname{dir}_{\phi}(B, x) \subset \operatorname{dir}(B, x) \subset \operatorname{dir}(A, x) \subset \operatorname{dir}(C, x) .
\end{aligned}
$$


Since both $\operatorname{dir}_{\phi}(A, x)$ and $\operatorname{dir}(C, x)$ are diametral for each $x \in B$, we conclude from (1) that

$$
\operatorname{dir}(B, x)=\operatorname{dir}_{\phi}(B, x)=\operatorname{dir}_{\phi}(A, x)=\operatorname{dir}(A, x)
$$

for each $x \in B$, and that $B$ is directional at each of its points.

6.18. Theorem. If $\phi \in \mathfrak{U}, \phi(A)<\infty, \mathfrak{D}_{\phi}^{\nabla}(A, x)<\infty$ for $\phi$ almost all $x$ in $A$, and $A$ is rectifiable, then $A$ contains a subset $B$ such that $\phi(A \widetilde{B})=0$ and such that $B$ is directional and $\phi$ directional at each of its points.

Proof. Use intersecting measure and 6.3, and apply Lemma 6.17.

6.19. Theorem. If $\phi \in \mathfrak{U}, \phi(A)<\infty, \mathfrak{D}_{\phi}^{\nabla}(A, x)<\infty$ for $\phi$ almost all $x$ in $A$, and $A$ is $\phi$ rectifiable, then corresponding to each positive number $\epsilon$ there is a set $B \subset A$ such that $\phi(A \widetilde{B})<\epsilon$ and such that $B$ is directional at each of its points.

Proof. Use 6.18 and the definition of $\phi$ rectifiability.

7. Restrictedness and rectifiability. We recall, before giving the next theorem and its proof, that $i$ is the point $(0,1)$ of $E_{2}$ and that for $x$ in $E_{2}$ the numbers $x_{1}$ and $x_{2}$ are such that $x=\left(x_{1}, x_{2}\right)$.

7.1. Theorem. If $\eta>0, A$ is a bounded subset of $E_{2}$, and $\Omega_{i}^{\eta} \operatorname{Sgn}(A, x)$ is vacuous whenever $x \in A$, then $A$ is rectifiable.

Proof. The theorem being obvious in case there is a $p \in E_{2}$ such that $A \subset\{p\}$, we assume that $A$ contains at least two points.

Let $p^{\prime}$ and $p^{\prime \prime}$ be distinct elements of $A$ and set $P=p^{\prime \prime}-p^{\prime}$. Since $\Re_{i}^{\eta} \operatorname{Sgn}(A, x)$ is vacuous for $x$ in $A$ we know that

$$
|i-P /| P|| \geqq \eta,
$$

from which follow successively the relations

$$
\begin{gathered}
1-P_{2} /|P|+\left|P_{1}\right| /|P| \geqq \eta \\
P_{2} \leqq\left|P_{1}\right|+(1-\eta)|P| \leqq\left|P_{1}\right|+(1-\eta)\left(\left|P_{1}\right|+\left|P_{2}\right|\right), \\
P_{2}-(1-\eta)\left|P_{2}\right| \leqq 2\left|P_{1}\right|
\end{gathered}
$$

Thus if $P_{2} \geqq 0$ we conclude from the last relation that

$$
P_{2} \leqq 2 \eta^{-1}\left|P_{1}\right|
$$

an inequality which is obvious if $P_{2}<0$. Accordingly

$$
p_{2}^{\prime \prime}-p_{2}^{\prime} \leqq 2 \eta^{-1}\left|p_{1}^{\prime \prime}-p_{1}^{\prime}\right|
$$

and we have shown

$$
x_{2}^{\prime \prime}-x_{2}^{\prime} \leqq 2 \eta^{-1}\left|x_{1}^{\prime \prime}-x_{1}^{\prime}\right| \text { for } x^{\prime} \in A \text { and } x^{\prime \prime} \in A \text {. }
$$

This implies 


$$
\left|x_{2}^{\prime \prime}-x_{2}^{\prime}\right| \leqq 2 \eta^{-1}\left|x_{1}^{\prime \prime}-x_{1}^{\prime}\right| \quad \text { for } x^{\prime} \text { and } x^{\prime \prime} \text { in } A
$$

which in turn implies

$$
\left|x_{2}^{\prime \prime}-x_{2}^{\prime}\right| \leqq 2 \eta^{-1}\left|x_{1}^{\prime \prime}-x_{1}^{\prime}\right| \quad \text { for } x^{\prime} \text { and } x^{\prime \prime} \text { in clsr } A .
$$

Letting

$$
\bar{A}=\operatorname{clsr} A, \quad C=\sum_{x \in \bar{A}}\left\{x_{1}\right\},
$$

we note that $C$ is a bounded closed subset of $E_{1}$ and that (1) assures us that $\bar{A}$ is the graph of a function (or, if we prefer, is a function) with domain $C$. Denoting this function by $h$ we observe

$$
\sum_{t \in C}\{(t, h(t))\}=\bar{A} .
$$

Taking $J$ as the closed interval containing $C$ with end points in $C$ we extend the function $h$ to the open intervals comprising $J-C$ by linear interpolation to obtain a function $g$ on $J$ which, because of (1), satisfies the Lipschitz condition

$$
\left|g\left(t^{\prime \prime}\right)-g\left(t^{\prime}\right)\right| \leqq 2 \eta^{-1}\left|t^{\prime \prime}-t^{\prime}\right| \quad \text { for } t^{\prime} \in J \text { and } t^{\prime \prime} \in J .
$$

Finally we define $f$ on $J$ by

$$
f(t)=(t, g(t))
$$

for $t \in J$.

Clearly $f$ satisfies the Lipschitz condition

$$
\left|f\left(t^{\prime \prime}\right)-f\left(t^{\prime}\right)\right| \leqq\left(1+2 \eta^{-1}\right)\left|t^{\prime \prime}-t^{\prime}\right| \quad \text { for } t^{\prime} \in J \text { and } t^{\prime \prime} \in J .
$$

By (2) the range of $f$ includes $\bar{A}$ which in turn includes $A$.

7.2. THEOREM. If $\eta>0, A$ is a bounded subset of $E_{2}, z \in E_{2}$ with $|z|=1$, and if $\Omega_{z}^{\eta} \operatorname{Sgn}(A, x)$ is vacuous whenever $x \in A$, then $A$ is rectifiable.

The proof is obvious and may be effected by the rotational device of letting

$$
\bar{S}=\sum_{x \in S}\{-i z x\},
$$

selecting $A_{0}$ so that $\bar{A}_{0}=A$, and checking that $A$ is congruent to the set $A_{0}$ which is rectifiable by 7.1 .

This scheme is employed in more detail in the proof of 7.19 where the argument is slightly more complicated due to the presence of a measure.

7.3. THEOREM. If $\eta>0, r>0, A$ is a bounded subset of $E_{2}, z \in E_{2}$ with $|z|=1$, and if $\Omega_{z}^{*} \operatorname{Sgn}\left(A \Omega_{x}^{r}, x\right)$ is vacuous whenever $x \in A$, then $A$ is rectifiable. 
Proof. Let $\mathfrak{F}$ be a finite family of sets such that $A \subset \sigma(\mathfrak{F}) \subset E_{2}$ and diam $s<2^{-1} r$ whenever $s \in \mathfrak{F}$. Clearly if $s \in \mathscr{F}$ and $x \in s$ then $A s \subset A \Omega_{x}^{r}$ and Sgn $(A s, x) \subset \operatorname{Sgn}\left(A \Omega_{x}^{r}, x\right)$. Thus by $7.2, s \in \mathfrak{F}$ implies $A s$ is rectifiable and therefore, $\mathfrak{F}$ being finite, $\sum_{s \in \mathfrak{F}}(A s)$ is rectifiable.

7.4. THEOREM. If $A$ is restricted at each of its points, then there is a countable family $\mathfrak{F}$ of rectifiable sets for which

$$
A=\sigma(\mathfrak{F}) .
$$

Proof. Recall 2.22. Let $\Gamma=E_{2} E_{z}[|z|=1]$. Let $B$ be such a countable subset of $\Gamma$ that its closure is $\Gamma$. Let $R$ be the set of positive rationals less than 1 . Let $N$ be the set of positive integers. Let $A_{n}=\Omega_{(0,0)}^{n} A$.

Checking that

$$
A=\sum_{z \in B} \sum_{\eta \in R} \sum_{r \in R} \sum_{n \in N} \omega(n, r, \eta, z)
$$

where

$$
\omega(n, r, \eta, z)=A_{n} \underset{x}{E}\left[\AA_{z}^{\prime} \operatorname{Sgn}\left(A_{n} \AA_{x}^{r}, x\right)=0\right],
$$

we see in the light of 7.3 that $\mathfrak{F}$ may be taken as the countable family

$$
\sum_{z \in B} \sum_{\eta \in R} \sum_{r \in R} \sum_{n \in N}\{\omega(n, r, \eta, z)\} .
$$

7.5. Definition. For $a$ and $\lambda$ positive numbers and $x \in E_{2}$ we define

$$
\langle x, a, \lambda\rangle=\underset{y}{E}\left[\left|y_{1}-x_{1}\right|<a \text { and }\left|y_{2}-x_{2}\right|<\lambda a\right] \text {. }
$$

7.6. Lemma. If $x \in E_{2}, 0<\lambda, 0<\eta, 0<a, \lambda \eta=40$, and if $x^{\prime}$ and $x^{\prime \prime}$ are elements of $\langle x, 5 a, \lambda\rangle$ for which $x_{2}^{\prime \prime}-x_{2}^{\prime}=\lambda a$, then there are sets $\alpha^{\prime}$ and $\alpha^{\prime \prime}$ such that

$$
\begin{gathered}
\alpha^{\prime}\left\{x^{\prime}\right\}+\alpha^{\prime \prime}\left\{x^{\prime \prime}\right\}=0, \quad \alpha^{\prime}+\alpha^{\prime \prime}=\langle x, 5 a, \lambda\rangle, \\
\operatorname{Sgn}\left(\alpha^{\prime}, x^{\prime}\right) \subset \Omega_{i}^{\prime \prime}, \quad \operatorname{Sgn}\left(\alpha^{\prime \prime}, x^{\prime \prime}\right) \subset \Omega_{-i}^{\prime \prime} .
\end{gathered}
$$

Proof. Consider the sets

$$
\begin{aligned}
\alpha^{\prime} & =\underset{y}{E}\left[\left|y_{1}-x_{1}\right|<5 a \text { and } \frac{x_{2}^{\prime}+x_{2}^{\prime \prime}}{2} \leqq y_{2}<x_{2}+5 \lambda a\right], \\
\alpha^{\prime \prime} & =\underset{y}{E}\left[\left|y_{1}-x_{1}\right|<5 a \text { and } x_{2}-5 \lambda a<y_{2} \leqq \frac{x_{2}^{\prime}+x_{2}^{\prime \prime}}{2}\right] .
\end{aligned}
$$

Clearly $\alpha^{\prime}\left\{x^{\prime}\right\}=0, \alpha^{\prime \prime}\left\{x^{\prime \prime}\right\}=0$ and $\alpha^{\prime}+\alpha^{\prime \prime}=\langle x, 5 a, \lambda\rangle$. We shall now prove

$$
\operatorname{Sgn}\left(\alpha^{\prime}, x^{\prime}\right) \subset \Omega_{i}^{\prime \prime}
$$

Let $p^{\prime} \in \alpha^{\prime}$, denote $p^{\prime}-x^{\prime}$ by $\xi^{\prime}$ and note the relations 


$$
\left|\xi^{\prime}\right| \geqq \xi_{2}^{\prime}=p_{2}^{\prime}-x_{2}^{\prime} \geqq \frac{x_{2}^{\prime}+x_{2}^{\prime \prime}}{2}-x_{2}^{\prime}=\frac{x_{2}^{\prime \prime}-x_{2}^{\prime}}{2}=\frac{\lambda a}{2}>0
$$

and

$$
\left|\xi_{1}^{\prime}\right|<10 a
$$

which together imply

$$
\begin{aligned}
\left|i-\frac{p^{\prime}-x^{\prime}}{\left|p^{\prime}-x^{\prime}\right|}\right| & =\left|i-\frac{\xi^{\prime}}{\left|\xi^{\prime}\right|}\right| \leqq 1-\frac{\xi_{2}^{\prime}}{\left|\xi^{\prime}\right|}+\frac{\left|\xi_{1}^{\prime}\right|}{\left|\xi^{\prime}\right|} \\
& =\frac{\left|\xi^{\prime}\right|-\xi_{2}^{\prime}+\left|\xi_{1}^{\prime}\right|}{\left|\xi^{\prime}\right|} \leqq \frac{\left|\xi_{1}\right|+\xi_{2}^{\prime}-\xi_{2}^{\prime}+\left|\xi_{1}^{\prime}\right|}{\left|\xi^{\prime}\right|} \\
& =\frac{2\left|\xi_{1}^{\prime}\right|}{\left|\xi^{\prime}\right|} \leqq \frac{4\left|\xi_{1}^{\prime}\right|}{\lambda a}<\frac{40 a}{\lambda a}=\frac{40}{\lambda}=\eta .
\end{aligned}
$$

Thus (1) is established.

Similarly taking $p^{\prime \prime} \in \alpha^{\prime \prime}$ and denoting $x^{\prime \prime}-p^{\prime \prime}$ by $\xi^{\prime \prime}$ we note the relations

$$
\left|\xi^{\prime \prime}\right| \geqq \lambda a / 2>0 \quad \text { and } \quad\left|\xi_{1}^{\prime \prime}\right|<10 a
$$

which together imply

$$
\left|-i-\frac{p^{\prime \prime}-x^{\prime \prime}}{\left|p^{\prime \prime}-x^{\prime \prime}\right|}\right|=\left|i-\frac{\xi^{\prime \prime}}{\left|\xi^{\prime \prime}\right|}\right|<\eta .
$$

We conclude

$$
\operatorname{Sgn}\left(\alpha^{\prime \prime}, x^{\prime \prime}\right) \subset \Omega_{-i}^{\prime \prime}
$$

As a corollary we have

7.7. Lemma. If $x \in E_{2}, 0<\lambda, 0<\eta, 0<a, \lambda \eta=40, y \in\langle x, 5 a, \lambda\rangle,\left|y_{2}-x_{2}\right|$ $=\lambda a$, then there are sets $\beta^{\prime}$ and $\beta^{\prime \prime}$ such that

$$
\begin{gathered}
\beta^{\prime}\{x\}+\beta^{\prime \prime}\{y\}=0, \quad \beta^{\prime}+\beta^{\prime \prime}=\langle x, 5 a, \lambda\rangle, \\
\operatorname{Sgn}\left(\beta^{\prime}, x\right)+\operatorname{Sgn}\left(\beta^{\prime \prime}, y\right) \subset{\Re^{\prime}-i}^{\eta}+\mathscr{R}_{i}^{\prime \prime} .
\end{gathered}
$$

7.8. Lemma. If $x \in A \subset E_{2}, 0<\eta<1, \lambda \eta=40, \omega=10^{-2}$, and $\operatorname{Sgn}(A, x) \cdot \mathbb{R}_{1}^{\omega \eta}$ is nonvacuous, then there is a number $b>0$ such that

$$
0 \neq A \underset{\nu}{E}\left[\left(\left|y_{1}-x_{1}\right|<b\right) \text { and }\left(\left|y_{2}-x_{2}\right|=\lambda b\right)\right] \text {. }
$$

Proof. Since Sgn $(A, x) \Re_{i}^{\omega \eta}$ is nonvacuous we select a point $p \in A$ such that

$$
\left|\frac{p-x}{|p-x|}-i\right|<\omega \eta<10^{-2}
$$


let $\xi=p-x$, and check in order:

$$
\begin{gathered}
\left|\frac{\xi}{|\xi|}-i\right|=\left|\frac{\xi_{1}}{|\xi|}+i\left(\frac{\xi_{2}}{|\xi|}-1\right)\right|<\omega \eta<10^{-2}, \\
\left|\frac{\xi_{2}}{|\xi|}-1\right|<10^{-2}, \quad\left|\xi_{2}\right|>0, \\
\left|\xi_{1}\right|<\omega \eta|\xi| \leqq \omega \eta\left|\xi_{1}\right|+\omega \eta\left|\xi_{2}\right| \leqq \frac{\left|\xi_{1}\right|}{2}+\omega \eta\left|\xi_{2}\right|, \\
\left|\xi_{1}\right| \leqq 2 \omega \eta\left|\xi_{2}\right|=\frac{4}{5} \frac{\left|\xi_{2}\right|}{\lambda}<\frac{\left|\xi_{2}\right|}{\lambda} .
\end{gathered}
$$

Accordingly

$$
\left|p_{1}-x_{1}\right|<\frac{\left|p_{2}-x_{2}\right|}{\lambda}
$$

We now define $b$ so that $\lambda b=\left|p_{2}-x_{2}\right|$, note that $\left|p_{1}-x_{1}\right|<b$, and conclude

$$
A \underset{y}{E}\left[\left(\left|y_{1}-x_{1}\right|<b\right) \text { and }\left(\left|y_{2}-x_{2}\right|=\lambda b\right)\right]
$$

is nonvacuous (in fact $p$ is in it).

7.9. Definition. We say $A$ is countably $\phi$ rectifiable if and only if $\phi \in \mathfrak{U}$ and there is a countable family $\mathfrak{F}$ of rectifiable sets such that

$$
\phi[A-\sigma(\mathfrak{F})]=0 .
$$

It should be noted that $A$ is countably $\phi$ rectifiable whenever it is $\phi$ rectifiable. Furthermore if $\phi(A)<\infty$ then $A$ is countably $\phi$ rectifiable when and only when $A$ is $\phi$ rectifiable. It is likewise obvious that $\sigma(\mathcal{B})$ is countably $\phi$ rectifiable whenever $B$ is a countable family of countably $\phi$ rectifiable sets.

7.10. Notation. Whenever $\phi \in \mathfrak{U}$ the notation $\phi^{0}$ will be used to denote that function $\psi$ on $\varepsilon_{2}$ such that $s \subset E_{2}$ implies $\psi(s)$ is the infimum of numbers of the form $\phi(S)$ where $S$ is an open set containing $s$.

Thus from 3.12 it follows that $\phi^{0} \in \mathfrak{U}$ or $\phi^{0} \in \mathfrak{M}$ according as $\phi \in \mathfrak{U}$ or $\phi \in \mathfrak{M}$.

7.11. Theorem. If $\phi \in \mathfrak{U}, \epsilon>0,0<\eta<1, \lambda \eta=40, \delta>0,0<\rho \leqq 40^{-1} \delta$, $\xi \in A \subset E_{2}$, and if corresponding to each $x \in A$ there is a set $B$ such that

(i) $\left(\Omega_{-i}+\Omega_{i}^{\eta}\right) \operatorname{Sgn}(\widetilde{B}, x)=0$,

(ii) $\phi\left(B \Omega_{x}^{r}\right) \leqq 2 r \in$ for $0<r \leqq \delta$,

then there is a rectifiable set $D$ such that

$$
\phi^{0}\left(A \tilde{D} \AA_{\xi}^{\rho}\right) \leqq 240 \lambda \rho \epsilon .
$$

Proof. Let

$$
K=A \Re_{\xi}^{p},
$$




$$
\begin{aligned}
& \omega=10^{-2}, \\
& D=K \underset{x}{E}\left[\Omega_{i}^{\omega_{i}} \operatorname{Sgn}(K, x)=0\right], \\
& C=K \tilde{D} .
\end{aligned}
$$

From 7.1 it follows that $D$ is rectifiable.

Notice that

$$
D+C=K,
$$

and that verification (in Part IV below) of the relation

$$
\phi^{0}(C) \leqq 240 \lambda \rho \epsilon
$$

will complete the proof.

For $x \in C$ we define a numerical set $T_{x}$ as follows: $b \in T_{x}$ if and only if $b>0$ and

$$
0 \neq K \underset{y}{E}\left[\left(\left|y_{1}-x_{1}\right|<b\right) \text { and }\left(\left|y_{2}-x_{2}\right|=\lambda b\right)\right] \text {. }
$$

Accordingly for $x \in C$ : we use (2), (3), (4) and Lemma 7.8 to check that $T_{x} \neq 0$; with the aid of (6) and (1) we infer

$$
\lambda \sup T_{x} \leqq \operatorname{diam} K \leqq 2 \rho<\infty ;
$$

we select a number $a_{x} \in T_{x}$ such that

$$
0<a_{x} \leqq \sup T_{x} \leqq 2 a_{x}<\infty .
$$

Letting

$$
\mathfrak{W}=\sum_{x \in c}\left\{\underset{t}{E}\left[\left|t-x_{1}\right|<a_{x}\right]\right\}, \quad X=\sum_{x \in C}\left\{x_{1}\right\},
$$

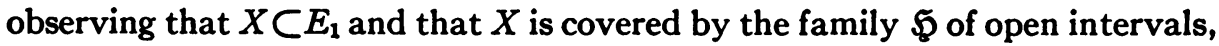
we use the one-dimensional analogue of 5.11 to obtain a countable subset $C_{0}$ of $C$ for which

$$
\sum_{x \in c_{0} \cdot}\left\{E\left[\left|t-x_{1}\right|<a_{x}\right]\right\} \text { is disjointed }
$$

and

$$
X \subset \sum_{x \in c_{0}} E\left[\left|t-x_{1}\right|<5 a_{x}\right]
$$

We divide the remainder of the proof into four parts.

PART I.

$$
\sum_{x \in c_{0}} 2 a_{x} \leqq 6 \rho
$$


Proof. With the aid of (4) and (1) we obtain

$$
\operatorname{diam} X \leqq \operatorname{diam} C \leqq \operatorname{diam} K \leqq 2 \rho
$$

so that we may use (8) and (7) to infer

$$
\operatorname{diam} \sum_{x \in c_{0}} E\left[\left|t-x_{1}\right|<a_{x}\right] \leqq 2 \rho+\operatorname{diam} X+2 \rho \leqq 6 \rho .
$$

Recourse to (9) completes this proof.

PART II.

$$
C \subset \sum_{x \in c_{0}}\left\langle x, 5 a_{x}, \lambda\right\rangle .
$$

Proof (by contradiction). Suppose $y$ is in $C$ and

$$
y \in \sum_{x \in c_{0}}\left\langle x, 5 a_{x}, \lambda\right\rangle,
$$

note that $y_{1} \in X$ and use (10) to secure an $x^{\prime}$ in $C$ for which

$$
\left|y_{1}-x_{1}^{\prime}\right|<5 a_{x^{\prime}} \text {. }
$$

Now the statement, $y \notin\left\langle x, 5 a_{x^{\prime}}, \lambda\right\rangle$, which is a consequence of (11), combines with (12) to yield

$$
5 \lambda a_{x^{\prime}} \leqq\left|y_{2}-x_{2}^{\prime}\right| \text {. }
$$

Determining $b$ so that $\lambda b=\left|y_{2}-x_{2}^{\prime}\right|$ we have

$$
5 \lambda a_{x^{\prime}} \leqq\left|y_{2}-x_{2}^{\prime}\right|=\lambda b .
$$

Thus (13) implies the relation

$$
5 a_{x^{\prime}} \leqq b<5 b
$$

which combines with (12) to tell us

$$
\left|y_{1}-x_{1}^{\prime}\right|<b .
$$

From the relations $y \in C \subset K,(14),(13),(6)$ follows the proposition

$$
b \in T_{x^{\prime}}
$$

which, viewed in the light of (8) and (13), implies the contradiction

$$
b \leqq \sup T_{x^{\prime}} \leqq 2 a_{x^{\prime}}<5 a_{x^{\prime}} \leqq b .
$$

PART III. If $x \in C_{0}$ then

$$
\phi\left(\left\langle x, 5 a_{x}, \lambda\right\rangle\right) \leqq 80 \lambda a_{x} \epsilon .
$$

Proof. Since $a_{x} \in T_{x}$ we use (6) to ascertain a point $y \in K$ with

$$
\left|y_{1}-x_{1}\right|<a_{x}, \quad\left|y_{2}-x_{2}\right|=\lambda a_{x} .
$$


Noting that $x$ and $y$ are in $\left\langle x, 5 a_{x}, \lambda\right\rangle$ with $\left|y_{2}-x_{2}\right|=\lambda a_{x}$, and invoking Lemma 7.7, we choose sets $\beta^{\prime}$ and $\beta^{\prime \prime}$ for which

$$
\begin{gathered}
\beta^{\prime}\{x\}+\beta^{\prime \prime}\{y\}=0, \quad \beta^{\prime}+\beta^{\prime \prime}=\left\langle x, 5 a_{x}, \lambda\right\rangle, \\
\operatorname{Sgn}\left(\beta^{\prime}, x\right)+\operatorname{Sgn}\left(\beta^{\prime \prime}, y\right) \subset \Omega_{-i}^{\prime \prime}+\dot{\Omega}_{i}^{\prime \prime} .
\end{gathered}
$$

Putting $d=\operatorname{diam}\left\langle x, 5 a_{x}, \lambda\right\rangle$, and checking that $1 \leqq \lambda<\infty$, we use (8) and (7) to obtain

$$
d \leqq 10 a_{x}+10 \lambda a_{x} \leqq 20 \lambda a_{x} \leqq 40 \rho \leqq \delta .
$$

Because of (16), (ii), (i), and the fact that $x$ and $y$ are in $K \subset A$, we can and do select sets $B_{1}$ and $B_{2}$ such that

$$
\phi\left(B_{1} \Omega_{x}^{d}\right) \leqq 2 d \epsilon, \quad \phi\left(B_{2} \Omega_{y}^{d}\right) \leqq 2 d \epsilon,
$$

and

$$
\left(\Omega_{-i}^{\prime}+\Omega_{i}^{\eta}\right)\left[\operatorname{Sgn}\left(\tilde{B}_{1}, x\right)+\operatorname{Sgn}\left(\tilde{B}_{2}, y\right)\right]=0 .
$$

Referring to (15) and the last relation we deduce

$$
\operatorname{Sgn}\left(\beta^{\prime} \tilde{B}_{1}, x\right)+\operatorname{Sgn}\left(\beta^{\prime \prime} \tilde{B}_{2}, y\right) \subset\left(\Omega_{-i}^{\eta}+\Omega_{i}^{\prime}\right)\left[\operatorname{Sgn}\left(\tilde{B}_{1}, x\right)+\operatorname{Sgn}\left(\tilde{B}_{2}, y\right)\right]=0,
$$

and thus, from 6.1 and (15), conclude $\beta^{\prime} \tilde{B}_{1}+\beta^{\prime \prime} \tilde{B}_{2} \subset \beta^{\prime}\{x\}+\beta^{\prime \prime}\{y\}=0$. Accordingly the relation

$$
\begin{aligned}
\left\langle x, 5 a_{x}, \lambda\right\rangle & =\beta^{\prime}+\beta^{\prime \prime}=\beta^{\prime} \tilde{B}_{1}+\beta^{\prime} B_{1}+\beta^{\prime \prime} \tilde{B}_{2}+\beta^{\prime \prime} B_{2} \\
& =\beta^{\prime} B_{1}+\beta^{\prime \prime} B_{2} \subset B_{1}\left\langle x, 5 a_{x}, \lambda\right\rangle+B_{2}\left\langle x, 5 a_{x}, \lambda\right\rangle \\
& \subset B_{1} \Omega_{x}^{d}+B_{2} \Omega_{y}^{d}
\end{aligned}
$$

may be combined with (17) and (16) to give

$$
\phi\left(\left\langle x, 5 a_{x}, \lambda\right\rangle\right) \leqq \phi\left(B_{1} \Omega_{x}^{d}\right)+\phi\left(B_{2} \Omega_{y}^{d}\right) \leqq 4 d \epsilon \leqq 80 \lambda a_{x} \epsilon .
$$

PART IV. $\phi^{0}(C) \leqq 240 \lambda \rho \epsilon$.

Proof. Using the fact that the set

$$
\sum_{x \in c_{0}}\left\langle x, 5 a_{x}, \lambda\right\rangle
$$

is open and also 7.10, Parts II, III, and I, we discover

$$
\begin{aligned}
\phi^{0}(C) & \leqq \phi\left(\sum_{x \in c_{0}}\left\langle x, 5 \hat{a}_{x}, \lambda\right\rangle\right) \leqq \sum_{x \in c_{0}} \phi\left(\left\langle x, 5 a_{x}, \lambda\right\rangle\right) \\
& \leqq \sum_{x \in c_{0}} 80 \lambda a_{x} \epsilon=40 \lambda \epsilon \sum_{x \in c_{0}} 2 a_{x} \leqq 240 \lambda \rho \epsilon .
\end{aligned}
$$

7.12. ThEOREM. If $\phi \in \mathfrak{U}, 0<\eta<1, \delta>0, A$ is a bounded subset of $E_{2}$, and if corresponding to each $x \in A$ there is a set $B$ such that 
(i) $\left(\Omega_{-i}^{\eta}+\Omega_{i}^{\eta}\right) \operatorname{Sgn}(\widetilde{B}, x)=0$,

(ii) $\phi\left(B \Omega_{x}^{r}\right) \leqq 2 r$ for $0<r \leqq \delta$, then there is a rectifiable set $C$ such that $\phi^{0}(A \tilde{C})<\infty$.

Proof. Let $\mathfrak{F}$ be a finite family of nonvacuous sets each with diameter less than $40^{-1} \delta$ such that $A=\sigma(\mathfrak{F})$. Letting $\lambda \eta=40, \rho=40^{-1} \delta, \epsilon=1$, we use 7.11 to associate with each $s \in \mathfrak{F}$ a point $x_{8} \in s$ and a rectifiable set $D_{s}$ such that

$$
\phi^{0}\left(s \tilde{D}_{s}\right)=\phi^{0}\left(s \tilde{D}_{s} \Omega_{x_{s}}^{\rho}\right) \leqq 240 \lambda \rho \epsilon<\infty .
$$

Accordingly the rectifiable set

$$
\sum_{s \in \mathfrak{F}} D_{s}
$$

has the properties demanded of $C$ by the theorem.

7.13. Theorem. If $\phi \in \mathfrak{M}, \phi(A)>0$,

$$
\mathfrak{D}_{\phi}^{\Delta}(x)>0 \text { and }(\{-i\}+\{i\}) \operatorname{dir}_{\phi}(x)=0
$$

for $\phi$ almost all $x \in A$, then there is a rectifiable set with positive $\phi$ measure.

Proof. Ascertain $\mu>0$ so that $\phi\left(A_{1}\right)>0$ where

$$
A_{1}=A \underset{x}{E}\left[\mathfrak{D}_{\phi}^{\Delta}(x)>\mu\right] \text {. }
$$

Remember that $\operatorname{Sgn}(s, x)$ is closed for $s \subset E_{2}$ and $x \in E_{2}$, and select a positive number $\eta<1$ such that $\phi\left(A_{2}\right)>0$ where $A_{2}$ is the set for which $x \in A_{2}$ if and only if there is a set $B$ such that

$$
x \in A_{1}, \quad\left(\Omega_{-i}^{\eta}+\Omega_{i}^{\eta}\right) \operatorname{Sgn}(\tilde{B}, x)=0, \quad \mathfrak{D}_{\phi}^{\Delta}(B, x)=0 .
$$

Choose $\lambda$ and $\epsilon$ so that

$$
\begin{aligned}
\lambda \eta & =40, \\
240 \lambda \epsilon & =\mu / 10 .
\end{aligned}
$$

Take $\delta>0$ so that $\phi\left(A_{3}\right)>0$ where $A_{3}$ is the set for which $x \in A_{3}$ if and only if there is a set $B$ such that

$$
\begin{gathered}
x \in A_{2}, \quad\left(\Re_{-i}^{\eta}+\Omega_{i}^{\eta}\right) \operatorname{Sgn}(\tilde{B}, x)=0, \\
\phi\left(B \Omega_{x}^{r}\right) \leqq 2 r \epsilon
\end{gathered}
$$$$
\text { for } 0<r \leqq \delta \text {. }
$$

Noting $A_{3} \subset A_{1}$ with $\phi\left(A_{3}\right)>0$, we infer that $\mathfrak{D}_{\phi}^{\Delta}(x)>\mu$ for $x \in A_{3}$, use 7.10 and 5.30 to obtain a point $\xi$ in $A_{3}$ for which $\mathfrak{D}_{\phi^{0}}^{\Delta}\left(A_{3}, \xi\right)>7^{-1} \mu$ and fix a number $\rho$ such that

$$
\begin{aligned}
0<\rho & \leqq \delta / 40, \\
\phi^{0}\left(A_{3} \Re_{\xi}^{\rho}\right) & >2 \mu \rho / 7>\mu \rho / 7 .
\end{aligned}
$$


With the aid of 7.11 we so secure a rectifiable set $D$ that

$$
\phi^{0}\left(A_{3} \tilde{D} \Omega_{\xi}^{p}\right) \leqq 240 \lambda \rho \epsilon .
$$

Viewing this last relation in the light of (2) and (1) we discover

$$
\begin{aligned}
\phi^{0}(D) & \geqq \phi^{0}\left(A_{3} D \Omega_{\xi}^{p}\right)=\phi^{0}\left(A_{3} \Omega_{\xi}^{p}\right)-\phi^{0}\left(A_{3} \tilde{D} \Omega_{\xi}^{p}\right) \\
& \geqq \mu \rho / 7-240 \lambda \rho \epsilon>\mu \rho / 10-\mu \rho / 10=0 .
\end{aligned}
$$

Now let $K=\operatorname{clsr} D$. Since $D$ is rectifiable, $K$ is likewise. From 3.12 and (3) follows the relation

$$
\phi(K)=\phi^{0}(K) \geqq \phi^{0}(D)>0 .
$$

Consequently there is a rectifiable set, namely $K$, with positive $\phi$ measure. The proof is complete.

7.14. TheOREM. If $\phi \in \mathfrak{M}, \phi(A)>0$,

$$
\mathfrak{D}_{\phi}^{\Delta}(x)>0 \text { and }(\{-i\}+\{i\}) \operatorname{dir}_{\phi}(x)=0
$$

for $\phi$ almost all $x \in A$, then there is a rectifiable subset of $A$ with positive $\phi$ measure.

Proof. Let $\Phi \in \mathfrak{M}$ be the function on $\varepsilon_{2}$ such that $s \subset E_{2}$ implies $\Phi(s)$ $=\phi(A s)$. Since $\mathfrak{D}_{\Phi}^{\Delta}(x)=\mathfrak{D}_{\phi}^{\Delta}(A, x)$ for $x \in E_{2}$ we infer from 5.32 that $\mathfrak{D}_{\mathbf{\phi}} \hat{\mathrm{A}}(x)>0$ for $\phi$ almost all $x$ in $A$. Inasmuch as the relation

$$
\operatorname{dir}_{\Phi}(x) \subset \operatorname{dir}_{\phi}(x) \quad \text { for } x \in E_{2}
$$

follows immediately from Definition 2.17 , it is evident that

$$
(\{-i\}+\{i\}) \operatorname{dir}_{\Phi}(x)=0
$$

for $\phi$ almost all $x \in A$. Consequently

$$
\mathfrak{D}_{\Phi}^{\Delta}(x)>0, \quad(\{-i\}+\{i\}) \operatorname{dir}_{\Phi}(x)=0
$$

not only for $\phi$ almost all $x$ in $A$ but for $\Phi$ almost all $x$.

Thus 7.13 guarantees us a rectifiable set $C$ with $\Phi(C)>0$ and we obtain the rectifiable set $A C$ with $\phi(A C)=\Phi(C)>0$.

7.15. LEMMA. If $\phi \in \mathfrak{M}$ and if every Borel set with positive $\phi$ measure contains a rectifiable set with positive $\phi$ measure, then $E_{2}$ is $\phi$ rectifiable.

Proof. Let $\epsilon>0$ and $\mathfrak{F}$ be the family of all rectifiable sets. Denote

$$
\sup _{\alpha \in \mathfrak{Z}} \phi(\alpha)
$$

by $M$ and let $C_{1} \subset C_{2} \subset C_{3} \subset \cdots$ be closed rectifiable sets for which $\phi\left(C_{n}\right) \rightarrow M$ as $n \rightarrow \infty$. Setting

$$
C=C_{1}+C_{2}+C_{3}+\cdots
$$


we observe that $C$ is a Borel set and that, whether $\phi(\tilde{C})>0$ or $\phi(\tilde{C})=0$, our hypotheses enable us to select a number $\mu>0$ and a rectifiable subset $A_{0}$ of $\widetilde{C}$ such that $\phi\left(A_{0}\right)=\mu \phi(\tilde{C})$. Since $C_{n}+A_{0}$ is then rectifiable with $C_{n} A_{0} \subset C A_{0}$ $C C \tilde{C}=0$ for each positive integer $n$, we have

$$
M+\mu \phi(\tilde{C})=M+\phi\left(A_{0}\right)=\lim _{n \rightarrow \infty}\left[\phi\left(C_{n}\right)+\phi\left(A_{0}\right)\right]=\lim _{n \rightarrow \infty} \phi\left(C_{n}+A_{0}\right) \leqq M,
$$

from which it follows that

$$
\mu \phi(\tilde{C})=0, \quad \phi(\tilde{C})=0 .
$$

Inasmuch as $\phi\left(\tilde{C}_{n}\right) \rightarrow \phi(\widetilde{C})=0$ as $n \rightarrow \infty$ we know that $\phi\left(\widetilde{C}_{n}\right)<\epsilon$ for $n$ sufficiently large. Accordingly $E_{2}$ is $\phi$ rectifiable.

7.16. Тнеокем. If $\phi \in \mathfrak{M}, A \subset E_{2}$,

$$
\mathfrak{D}_{\phi}^{\Delta}(x)>0 \text { and }(\{-i\}+\{i\}) \operatorname{dir}_{\phi}(x)=0
$$

for $\phi$ almost all $x \in A$, then $A$ is $\phi$ rectifiable.

Proof. Let $\Phi \in \mathfrak{M}$ be the function on $\varepsilon_{2}$ such that $s \subset E_{2}$ implies $\Phi(s)$ $=\phi(A s)$. A glance at the proof of 7.14 convinces us that

$$
\mathfrak{D}_{\Phi}^{\Delta}(x)>0, \quad(\{-i\}+\{i\}) \operatorname{dir}_{\Phi}(x)=0
$$

for $\Phi$ almost all $x$. Thus, corresponding to each Borel set $B$ with positive $\Phi$ measure there is, by 7.14, a rectifiable subset of $B$ with positive $\Phi$ measure. Accordingly 7.15 assures us $E_{2}$ is $\Phi$ rectifiable. Hence $A$ is $\phi$ rectifiable.

7.17. Theorem. If $\phi \in \mathfrak{U}, \phi^{0}(A)<\infty$,

$$
\mathfrak{D}_{\phi}^{\Delta}(x)>0 \text { and }(\{-i\}+\{i\}) \operatorname{dir}_{\phi}(x)=0
$$

for $\phi$ almost all $x \in A$, then $A$ is $\phi$ rectifiable.

Proof. Since $\phi^{0}(A)<\infty$ we can and do select an open set $G$ containing $A$ for which $\phi(G)<\infty$. Letting $\Phi$ be the function on $\varepsilon_{2}$ such that $s \subset E_{2}$ implies $\Phi(s)=\phi(G s)$ we note $: \Phi \in \mathbb{M}$;

$$
\mathfrak{D}_{\Phi}^{\Delta}(x)=\mathfrak{D}_{\phi}^{\Delta}(x), \quad \operatorname{dir}_{\Phi}(x)=\operatorname{dir}_{\phi}(x)
$$

for each $x$ in the open set $G$. Consequently

$$
\mathfrak{D}_{\Phi}^{\Delta}(x)>0, \quad(\{-i\}+\{i\}) \operatorname{dir}_{\Phi}(x)=0
$$

for $\phi$ and $\Phi$ almost all $x \in A$. Theorem 7.16 now assures us that $A$ is $\Phi$ rectifiable. Thus $A$, being a subset of $G$, is $\phi$ rectifiable.

7.18. Theorem. If $\phi \in \mathfrak{U}, A \subset E_{2}$,

$$
\mathfrak{D}_{\phi}^{\Delta}(x)>0 \text { and }(\{-i\}+\{i\}) \operatorname{dir}_{\phi}(x)=0
$$


for $\phi$ almost all $x \in A$, then $A$ is countably $\phi$ rectifiable.

Proof. Let $R$ be the set of positive rationals less than 1. Let $N$ be the set of positive integers. For $n \in N, \eta \in R, \delta \in R$ we let $\omega(n, \eta, \delta)$ be the set defined by: $x \in \omega(n, \eta, \delta)$ if and only if there is a set $B$ such that

$$
\begin{gathered}
x \in A, \quad|x| \leqq n, \\
\left(\Omega_{-i}^{\eta}+\Omega_{i}^{\eta}\right) \operatorname{Sgn}(\widetilde{B}, x)=0, \\
\phi\left(B \Omega_{x}^{r}\right) \leqq 2 r
\end{gathered}
$$$$
\text { for } 0<r \leqq \delta \text {. }
$$

Setting

$$
H=\sum_{\delta \in R} \sum_{n \in R} \sum_{n \in N} \omega(n, \eta, \delta)
$$

we check that

$$
A \supset H \supset A \underset{x}{E}\left[(\{-i\}+\{i\}) \operatorname{dir}_{\phi}(x)=0\right]
$$

infer from our hypotheses that

$$
\phi(A \tilde{H})=0,
$$

and divide the remainder of the proof into two parts.

PART I. If $n \in N, \eta \in R, \delta \in R$, then $\omega(n, \eta, \delta)$ is countably rectifiable.

Proof. Let $D=\omega(n, \eta, \delta)$. Since (0) implies $D$ is a bounded subset of $A$, we use (1), (2), and 7.12 to obtain a rectifiable set $C$ such that $\phi^{0}(D \tilde{C})<\infty$. From 7.17 it follows that $D \tilde{C}$ is $\phi$ rectifiable. Thus the set $D$, which equals $D C+D \tilde{C}$, is $\phi$ rectifiable and hence, a fortiori, countably $\phi$ rectifiable.

PART II. $A$ is countably $\phi$ rectifiable.

Proof. From (3) and Part I we know $H$ is countably $\phi$ rectifiable. In view of (4) it is now evident that $A$ is countably $\phi$ rectifiable.

7.19. Theorem. If $\phi \in \mathfrak{U}, A \subset E_{2}, Z$ is a diametral set,

$$
\mathfrak{D}_{\phi}^{\Delta}(x)>0 \text { and } Z \operatorname{dir}_{\phi}(x)=0
$$

for $\phi$ almost all $x \in A$, then $A$ is countably $\phi$ rectifiable.

Proof. Let $\{-z\}+\{z\}=Z ; x^{\prime}=-z i x$, for $x \in E_{2}$;

$$
\bar{s}=\sum_{x \in \mathcal{s}}\left\{x^{\prime}\right\}
$$

for $s \subset E_{2}$. Let $\psi$ be the function on $\varepsilon_{2}$ such that

$$
\psi(s)=\phi(\bar{s})
$$

for $s \subset E_{2}$.

Observe that $\left|x^{\prime}-y^{\prime}\right|=|x-y|$ for $x$ and $y$ in $E_{2}$, check that $\psi \in \mathfrak{U}$, and note that 


$$
\overline{\sum_{s \in \mathfrak{F}} s}=\sum_{s \in \mathfrak{F}} \bar{s}, \quad \overline{\prod_{s \in \mathfrak{F}} s}=\prod_{\mathfrak{s} \in \mathfrak{F}} s
$$

for each family $\mathfrak{F}$ of subsets of $E_{2}$. Next infer

$$
\begin{aligned}
& \overline{\operatorname{Sgn}(s, x)}=\operatorname{Sgn}\left(\bar{s}, x^{\prime}\right), \quad \mathfrak{D}_{\psi}^{\Delta}(s, x)=\mathfrak{D}_{\phi}^{\Delta}\left(\bar{s}, x^{\prime}\right), \\
& \overline{\operatorname{dir}_{\psi}(x)}=\operatorname{dir}_{\phi}\left(x^{\prime}\right)
\end{aligned}
$$

whenever $s \subset E_{2}$ and $x \in E_{2}$.

Setting

$$
A_{1}=A \underset{y}{E}\left[\mathfrak{D}_{\phi}^{\Delta}(y)>0 \text { and } Z \operatorname{dir}_{\phi}(y)=0\right]
$$

we select $A_{0}$ so that $\bar{A}_{0}=A_{1}$ and verify that

$$
\overline{(\{-i\}+\{i\}) \operatorname{dir}_{\psi}(x)}=(\{-z\}+\{z\}) \operatorname{dir}_{\phi}\left(x^{\prime}\right)=Z \operatorname{dir}_{\phi}\left(x^{\prime}\right)=0
$$

for $x \in A_{0}$. With the help of (2), (1), (3), 7.18 and 7.9 we secure a countable family $(B)$ of rectifiable sets such that

$$
\psi\left[A_{0}-\sigma(\xi)\right]=0
$$

Consequently

$$
0=\phi\left[\bar{A}_{0}-\overline{\sigma(\xi)}\right]=\phi\left[A_{1}-\sum_{s \in \Theta} \bar{s}\right] .
$$

Since $\bar{s}$ is congruent to the rectifiable set $s$ for each $s \in \mathbb{B}$ it is now clear that $A_{1}$ is countably $\phi$ rectifiable and, inasmuch as our hypotheses and (2) together imply $\phi\left(A \tilde{A}_{1}\right)=0$, it is evident that $A$ is likewise countably $\phi$ rectifiable.

7.20. THEOREM. If $\phi \in \mathfrak{U}$ and $E_{2}$ is $\phi$ restricted at $\phi$ almost all of its points then $E_{2}$ is countably $\phi$ rectifiable.

Proof. Letting

$$
\Gamma=E_{2} \underset{z}{E}[|z|=1], \quad \alpha(z)=\underset{x}{E}\left[(\{-z\}+\{z\}) \operatorname{dir}_{\phi}(x)=0\right],
$$

we select a countable subset $\beta$ of $\Gamma$ whose closure is $\Gamma$. Since $\operatorname{dir}_{\phi}(x)$ is closed for $x \in E_{2}$ it is clear that $\phi(\widetilde{B})=0$ where

$$
B=\sum_{z \in \beta} \alpha(z) \text {. }
$$

Now for $z \in \beta$ we gather from 2.24 that $\mathfrak{D}_{\phi}^{\Delta}(x)>0$ for $\phi$ alnıost all $x$ in $\alpha(z)$ and hence conclude from 7.19 that $\alpha(z)$ is countably $\phi$ rectifiable.

Accordingly $B$, and hence $E_{2}$, is.countably $\phi$ rectifiable.

7.21. TheOREM. If $\phi \in \mathfrak{U}$, and $A$ is $\phi$ restricted at $\phi$ almost all of its points then $A$ is countably $\phi$ rectifiable. 
Proof. Let $\Phi \in \mathfrak{U}$ be the function on $\varepsilon_{2}$ such that $\Phi(s)=\phi(A s)$ for $s \subset E_{2}$. With the help of 6.5 (iii), 6.7, 6.3, we deduce

$$
\operatorname{dir}_{\Phi}(x)=\operatorname{dir}_{\Phi}(A, x)+\operatorname{dir}_{\Phi}(\tilde{A}, x)=\operatorname{dir}_{\Phi}(A, x)=\operatorname{dir}_{\phi}(A, x)
$$

for $x \in A$. Checking that $\mathfrak{D}_{\Phi}^{\Delta}(x)=\mathfrak{D}_{\phi}^{\Delta}(A, x)$ for $x \in E_{2}$ it follows from Definition 2.24 and our hypotheses that $E_{2}$ is $\Phi$ restricted not only for $\phi$ almost all $x \in A$ but for $\Phi$ almost all $x$. Accordingly Theorem 7.20 implies $E_{2}$ is countably $\Phi$ rectifiable. That $A$ is countably $\phi$ rectifiable is now evident.

7.22. Theorem. If $\phi \in \mathfrak{U}, \phi(A)<\infty$, and $A$ is $\phi$ restricted at $\phi$ almost all of its points then $A$ is $\phi$ rectifiable.

Proof. Use 7.21 and the fact that $\phi(A)<\infty$.

7.23. Theorem. If $\phi \in \mathfrak{U}$, and $A$ is countably $\phi$ rectifiable then $\mathfrak{D}_{\phi}^{\nabla}(A, x)>0$ for $\phi$ almost all $x \in A$.

Proof. Introduce intersecting measure and use 5.28.

7.24. TheOREM. If $\phi \in \mathfrak{U}, B$ is $\phi$ restricted at $\phi$ almost all of its points, $A \subset B$, then $A$ is $\phi$ restricted at $\phi$ almost all of its points.

Proof. Inasmuch as $\operatorname{dir}_{\phi}(A, x) \subset \operatorname{dir}_{\phi}(B, x)$ for $x \in E_{2}$, the only difficulty arises in verifying that $\mathfrak{D}_{\phi}^{\Delta}(A, x)>0$ for $\phi$ almost all $x \in A$.

Since $B$ is countably $\phi$ rectifiable by 7.21 , we see at once that $A$ is likewise countably $\phi$ rectifiable. From 7.23 it is apparent that

for $\phi$ almost all $x \in A$.

$$
\mathfrak{D}_{\phi}^{\Delta}(A, x) \geqq \mathfrak{D}_{\phi}^{\nabla}(A, x)>0
$$

7.25. TheOREM. If $\phi \in \mathfrak{U}$, and $A$ is restricted at each of its points then $A$ is countably $\phi$ rectifiable.

Proof. Use 7.4.

7.26. Theorem. If $\phi \in \mathfrak{U}, \phi(A)<\infty$, and $A$ is restricted at each of its points, then $A$ is $\phi$ rectifiable.

Proof. Use 7.25.

7.27. Theorem. If $A$ is $\phi$ directional at $x$, then $A$ is $\phi$ restricted at $x$.

Proof. Letting $\{-z\}+\{z\}=\operatorname{dir}_{\phi}(A, x)$, we note that $\{-i z\}+\{i z\}$ is a diametral set whose intersection with $\operatorname{dir}_{\phi}(A, x)$ is vacuous. Moreover inasmuch as $\operatorname{dir}_{\phi}(A, x) \neq 0$ it follows from 6.7 that $\mathfrak{D}_{\phi}^{\Delta}(A, x)>0$.

Obviously we also have

7.28. Theorem. If $A$ is directional at $x$, then $A$ is restricted at $x$.

7.29. TheOREM. If $\phi \in \mathfrak{U}, \phi(A)<\infty$, and if corresponding to each positive 
number $\epsilon$ there is a set $B C A$ such that $\phi(A \widetilde{B})<\epsilon$ and such that $B$ is restricted at each of its points, then $A$ is $\phi$ rectifiable.

Proof. See 7.26.

7.30. REMARK. If in Theorem 7.21 we replace $\phi$ by counting measure, the theorem which emerges is noticeably weaker than 7.4. Thus in view of the meaning (see 2.22) of the phrase " $A$ is restricted at $x$ " it is natural to regard the phrase " $\mathrm{A}$ is $\phi$ restricted at $x$ " as short (compare with 2.24) for:

$$
\mathfrak{D}_{\phi}^{\Delta}(A, x)>0
$$

and there is a point $z \in E_{2}$ with $|z|=1$ for which

$$
\{z\} \operatorname{dir}_{\phi}(A, x)=0 \text {. }
$$

The futility of this viewpoint will now be shown.

Throughout this remark we shall call a set a triangle if and only if it is a closed set consisting of the interior and boundary of an equilateral triangle. with its base horizontal and below the opposite vertex. Thus the length of each of the three sides of a triangle is equal to the diameter of the triangle.

For $T$ a triangle and $n$ a positive integer: construct $2 n+1$ triangles, each with diameter equal to

$$
\frac{\operatorname{diam} T}{2 n+1},
$$

so placed that the union of their bases is the base of $T$; next take the middle triangle and vertically translate it upward until its top vertex coincides with the top vertex of $T$; denote the family of $2 n+1$ triangles thus obtained by $O_{n}(T)$.

Let $T_{0}$ be a triangle with diameter 1 . Let $\mathfrak{F}_{0}=\left\{T_{0}\right\}$. Let $\mathfrak{F}_{1}, \mathfrak{F}_{2}, \mathfrak{F}_{3}, \cdots$ be so determined that

$$
\mathfrak{F}_{n}=\sum_{T \in \mathfrak{F}_{n-1}} O_{n}(T)
$$

for each positive integer $n$.

Noting that $\sigma\left(\mathfrak{F}_{1}\right) \supset \sigma\left(\mathfrak{F}_{2}\right) \supset \sigma\left(\mathfrak{F}_{3}\right) \supset \ldots$ we define

$$
\mathfrak{F}=\mathfrak{F}_{1}+\mathfrak{F}_{2}+\mathfrak{F}_{3}+\cdots
$$

and $\left({ }^{2}\right)$

$$
\mathcal{A}=\prod_{n=1}^{\infty} \sigma\left(\mathfrak{F}_{n}\right)
$$

(2) This set $A$ seems to have many properties in common with the set $P$ described on pages 327 and 328 of [1]. 
Since $T \in \mathfrak{F}$ implies the projection of $\mathcal{A T}$ on the base of $T$ is the base of $T$ we have little difficulty in verifying that

$$
L(\mathcal{A} T)=\operatorname{diam} T
$$

for $T \in \mathfrak{F}$

In particular

$$
L(\mathcal{A})=1 .
$$

An appraisal of our geometric construction convinces us that

$$
\left\{e^{i \theta}\right\} \operatorname{dir}_{L}(\mathcal{A}, x)=0
$$

for $x \in \mathcal{C}$ and $\pi / 3<\theta<2 \pi / 3$. In fact a more careful analysis reveals that

$$
\left\{e^{i \theta}\right\} \operatorname{dir}_{L}(\mathcal{A}, x)=0
$$

for $x \in \mathcal{C} A$ and $0<\theta<\pi$.

We shall term a triangle in $\mathfrak{F}$ an apex triangle if and only if its top vertex coincides with the top vertex of some larger triangle in $\mathfrak{F}$.

Let $W$ be the set so defined that $x \in W$ if and only if $x \in \mathcal{A}$ and the number of apex triangles of which $x$ is an element is finite. Inasmuch as

$$
\prod_{n=1}^{\infty}\left(1-\frac{1}{2 n+1}\right)=0 \text {, }
$$

we conclude $L(W)=0$.

A check shows that

$$
\mathfrak{D}_{L}^{\nabla}(\mathcal{A}, x)=0
$$

for $x \in \mathcal{A} \tilde{W}$;

consequently $\mathfrak{D}_{L}^{\nabla}(\mathcal{A}, x)=0$ for $L$ almost all $x$ in $\mathcal{A}$.

Since $L(\mathcal{A})=1>0$ it follows from 11.1 (1) and 11.1 (4) that $\mathcal{A}$ is not $L$ rectifiable.

A further examination of $\mathcal{A}$ makes clear that

$$
\mathfrak{D}_{L}^{\Delta}(\mathcal{A}, x) \geqq 1 / 2 \quad \text { for } x \in \mathcal{A} ;
$$

and a closer scrutiny discloses that

$$
\mathfrak{D}_{L}^{\Delta}(\mathcal{A}, x)=1
$$

for $L$ almost all $x$ in $\mathcal{A}$.

Now consider the two statements which follow.

Statement I. If $\phi \in \mathfrak{U}, \phi(A)<\infty$,

$$
\mathfrak{D}_{\phi}^{\Delta}(A, x)>0 \text { and } \quad(\{-i\}+\{i\}) \operatorname{dir}_{\phi}(A, x)=0
$$

for $\phi$ almost all $x$ in $A$, then $A$ is $\phi$ rectifiable.

Statement II. If $\phi \in \mathfrak{U}, \phi(A)<\infty$, 


$$
\mathfrak{D}_{\phi}^{\Delta}(A, x)>0 \text { and }\{i\} \operatorname{dir}_{\phi}(A, x)=0
$$

for $\phi$ almost all $x$ in $A$, then $A$ is $\phi$ rectifiable.

Since $e^{i \pi / 2}=i$, it follows from 7.22 and the properties of $\mathcal{A}$ that Statement $I$ is true and Statement II is false.

8. Density ratios. Recalling abbreviation 2.27 we state

8.1. Theorem. If $\psi \in \mathfrak{M}, P=(0,0), Q=(1,0), A \subset B \subset E_{2}$, and if further

(i) $P \in A$ and $Q \in A$,

(ii) $1 \leqq \psi\left(\Omega_{x}^{r}\right) / 2 r \leqq 1.01$ for $x \in B$ and $0<r \leqq 2$,

(iii) $1 \leqq \psi\left(B \Omega_{x}^{r}\right) / 2 r \leqq 1.01$ for $x \in A$ and $0<r \leqq 2$, then $\psi(P * Q)>.06$.

Proof. Let

$$
\begin{aligned}
& \Gamma=\Omega_{P}^{1}, \quad \rho=(2.4)^{1 / 2}, \quad \Gamma_{1}^{\prime}=\Omega_{P}^{\rho}, \\
& \Gamma_{2}=\Omega_{Q}^{1}, \quad \Gamma_{2}^{\prime}=\Omega_{Q}^{.6}, \quad \Gamma_{2}^{\prime \prime}=\Omega_{Q}^{2}, \\
& R=(1.6,0), \quad \Gamma_{3}=\Omega_{R}^{1.4}, \quad H=\Gamma_{2}^{\prime}-\Gamma_{1}^{\prime} .
\end{aligned}
$$

It is possible that the reader may find it instructive to draw a judicious number of the above circles. However the reasoning here given is purely arithmetic in form.

In order to prove $\psi(P * Q)>.06$ we assume hereinafter that $\psi(P * Q) \leqq .06$ and divide the remainder of the argument into parts culminating in Part VI which is easily recognized as false.

PART I. $\psi\left(\Gamma_{1} \Gamma_{2}\right) \leqq .06$.

Proof. $\Gamma_{1} \Gamma_{2}=P * Q$.

PART II. $\Gamma_{1}+\Gamma_{2}+\Gamma_{3} \subset \Gamma_{2}^{\prime \prime}$.

Proof. $x \in \Gamma_{1}$ implies

$$
|x-Q| \leqq|x-P|+|P-Q|<1+1=2
$$

which in turn implies $x \in \Gamma_{2}^{\prime \prime}$.

Obviously $\Gamma_{2} \subset \Gamma_{2}^{\prime \prime}$.

Finally $x \in \Gamma_{8}$ implies

$$
|x-Q| \leqq|x-R|+|R-Q|<1.4+.6=2 \text {, and } x \in \Gamma_{2}^{\prime \prime} .
$$

PART III. $\Gamma_{3} \Gamma_{1} \subset \Gamma_{2} \Gamma_{1}$.

Proof. Suppose $x \in \Gamma_{3} \Gamma_{1}$ and let $r_{1}=|x-P|, r_{2}=|x-Q|, r_{3}=|x-R|$. Since $x \in \Gamma_{3} \Gamma_{1}$ we know

$$
r_{1}^{2}<1, \quad r_{3}^{2}<(1.4)^{2}=49 / 25
$$

We also have

$$
x_{1}^{2}+x_{2}^{2}=r_{1}^{2}
$$




$$
\begin{gathered}
\left(x_{1}-8 / 5\right)^{2}+x_{2}^{2}=\left(x_{1}-1.6\right)^{2}+x_{2}^{2}=r_{3}^{2}, \\
r_{2}^{2}=\left(x_{1}-1\right)^{2}+x_{2}^{2} .
\end{gathered}
$$

From (2) and (3) we find

$$
80 x_{1}=25 r_{1}^{2}-25 r_{3}^{2}+64 .
$$

From (4) and (2) we have

$$
40 r_{2}^{2}=40+40 r_{1}^{2}-80 x_{1} .
$$

Thus

$$
\begin{aligned}
40 r_{2}^{2} & =40+40 r_{1}^{2}-25 r_{1}^{2}+25 r_{3}^{2}-64 \\
& =-24+15 r_{1}^{2}+25 r_{3}^{2} .
\end{aligned}
$$

Use of (1) yields

$$
40 r_{2}^{2}<-24+15+25(49 / 25)=40 .
$$

Accordingly $r_{2}<1$ and $x \in \Gamma_{2}$, and since $x \in \Gamma_{1}$, we have shown that $x \in \Gamma_{2} \Gamma_{1}$. The proof of Part III is complete.

Part IV. If $x \in H$, then $|x-R|<.31$.

Proof. Let $r_{1}=|x-P|, r_{2}=|x-Q|, r_{3}=|x-R|$. Since $x \in H=\Gamma_{2}^{\prime}-\Gamma_{1}^{\prime}$ we know

$$
r_{2}^{2}<.36, \quad r_{1}^{2} \geqq 2.4
$$

We also know

$$
\begin{gathered}
x_{1}^{2}+x_{2}^{2}=r_{1}^{2}, \\
\left(x_{1}-1\right)^{2}+x_{2}^{2}=r_{2}^{2}, \\
r_{3}^{2}=\left(x_{1}-1.6\right)^{2}+x_{2}^{2} .
\end{gathered}
$$

From (6) and (7) we find

$$
2 x_{1}=r_{1}^{2}-r_{2}^{2}+1
$$

From (8) and (6) we have

$$
r_{3}^{2}=2.56+r_{1}^{2}-3.2 x_{1}
$$

Thus

$$
\begin{aligned}
r_{3}^{2} & =2.56+r_{1}^{2}-1.6 r_{1}^{2}+1.6 r_{2}^{2}-1.6 \\
& =.96+1.6 r_{2}^{2}-.6 r_{1}^{2} .
\end{aligned}
$$

Use of (5) yields

$$
r_{3}^{2}<.96+(1.6)(.36)-(.6)(2.4)=.096<(.31)^{2} .
$$


The proof of Part IV is complete.

PART V. The set $B H$ is vacuous.

Proof (by contradiction). Suppose $x \in B H$ and let $r_{3}=|x-R|$. By Part IV we know

$$
r_{3}<.31
$$

Now let

$$
\Gamma=\Omega_{x}^{1.4-r_{2}}
$$

and note that $y \in \Gamma$ implies

$$
|y-R| \leqq|y-x|+|x-R|<1.4-r_{3}+r_{3}=1.4,
$$

which in turn implies $y \in \Gamma_{3}$. Thus $\Gamma \subset \Gamma_{3}$ and, using Part III, we infer that

$$
\Gamma \Gamma_{1} \subset \Gamma_{3} \Gamma_{1} \subset \Gamma_{2} \Gamma_{1}
$$

and also, making use of Part II, that

$$
\Gamma_{2}^{\prime \prime} \supset \Gamma_{1}+\Gamma_{3} \supset \Gamma_{1}+\Gamma \text {. }
$$

Hence from (ii), (10), and Part I we obtain

$$
\begin{aligned}
4.04 & \geqq \psi\left(\Gamma_{2}^{\prime \prime}\right) \geqq \psi\left(\Gamma_{1}+\Gamma\right)=\psi\left(\Gamma_{1}\right)+\psi(\Gamma)-\psi\left(\Gamma \Gamma_{1}\right) \\
& \geqq 2+2.8-2 r_{3}-\psi\left(\Gamma_{2} \Gamma_{1}\right) \geqq 4.8-2 r_{3}-.06 .
\end{aligned}
$$

Thus $4.04 \geqq 4.74-2 r_{3}$. That is,

$$
r_{3} \geqq 2.37-2.02=.35
$$

in contradiction to (9).

PART VI. $3.131>3.14$.

Proof. Since $H=\Gamma_{2}^{\prime}-\Gamma_{1}^{\prime}$ we have

$$
\Gamma_{2}^{\prime}=\Gamma_{2}^{\prime} \Gamma_{1}^{\prime}+\left(\Gamma_{2}^{\prime}-\Gamma_{1}^{\prime}\right)=\Gamma_{2}^{\prime} \Gamma_{1}^{\prime}+H,
$$

and since $B H$ is vacuous by $\operatorname{Part} \mathrm{V}$, we see that

$$
B \Gamma_{2}^{\prime}=B \Gamma_{2}^{\prime} \Gamma_{1}^{\prime} \text {. }
$$

Therefore $B \Gamma_{1}^{\prime} \supset B \Gamma_{2}^{\prime}$ and hence

$$
B \Gamma_{1}^{\prime} \supset B \Gamma_{1}^{\prime}+B \Gamma_{2}^{\prime} \supset B \Gamma_{1}+B \Gamma_{2}^{\prime},
$$

inasmuch as $\Gamma_{1} \subset \Gamma_{1}^{\prime}$. Using (iii), the fact that $\Gamma_{2}^{\prime} \subset \Gamma_{2}$, and Part I, we infer

$$
\begin{aligned}
2.02 \rho & \geqq \psi\left(B \Gamma_{1}^{\prime}\right) \geqq \psi\left(B \Gamma_{1}+B \Gamma_{2}^{\prime}\right) \\
& =\psi\left(B \Gamma_{1}\right)+\psi\left(B \Gamma_{2}^{\prime}\right)-\psi\left(B \Gamma_{1} \Gamma_{2}^{\prime}\right) \\
& \geqq \psi\left(B \Gamma_{1}\right)+\psi\left(B \Gamma_{2}^{\prime}\right)-\psi\left(\Gamma_{1} \Gamma_{2}\right) \\
& \geqq 2+1.2-.06 .
\end{aligned}
$$


Consequently $2.02 \rho \geqq 3.14$. Remembering that $\rho=(2.4)^{1 / 2}<1.55$ we get

$$
3.131=(2.02)(1.55)>2.02 \rho \geqq 3.14 \text {. }
$$

The proof of Part VI is complete.

8.2. ThEOREM. If $\phi \in \mathfrak{M}, \phi\left(E_{2}\right)>0$, and $\mathfrak{D}_{\phi}^{\Delta}(x)<1.01 \mathfrak{D}_{\phi}^{\nabla}(x)$ for $\phi$ almost all $x$, then there is a closed set $C$ with $\phi(C)>0$ and $a$ number $\mu>0$ such that

$$
\phi(p * q) \geqq \mu|q-p|
$$

for $p \in C$ and $q \in C$.

Proof. In accordance with 5.9 we select positive numbers $\alpha$ and $\delta_{1}$ and a bounded closed set $D$ with $\phi(D)>0$ such that

$$
\alpha \leqq \frac{\phi\left(\Omega_{\xi}^{\rho}\right)}{2 \rho} \leqq 1.01 \alpha<1.01 D_{\phi}^{\nabla}(\xi)
$$

for $0<\rho<\delta_{1}$ and $\xi \in D$. Letting $\Phi \in \mathfrak{M}$ be the function on $\varepsilon_{2}$ such that

$$
\Phi(s)=\phi(D s) \quad \text { for } s \subset E_{2},
$$

and checking that $\mathfrak{D}_{\hat{\Phi}}^{\hat{\phi}}(\xi) \leqq \mathfrak{D}_{\phi}^{\Delta}(\xi)<\infty$ for $\phi$ and $\Phi$ almost all $\xi$ we are assured by 5.14 and 5.26 that

$$
\alpha<\mathfrak{D}_{\phi}^{\nabla}(\xi)=\mathfrak{D}_{\phi}^{\nabla}(D, \xi)=\mathfrak{D}_{\Phi}^{\nabla}(\xi)
$$

for $L$ and $\Phi$ almost all $\xi$ in $D$. Inasmuch as $\Phi(D)=\phi(D)>0$, Lemma 5.5 yields us a number $\delta_{2}>0$ and a bounded closed set $D_{0}$ such that

$$
\Phi\left(D_{0}\right)>0, \quad \alpha \leqq \frac{\Phi\left(\Omega_{\xi}^{\rho}\right)}{2 \rho},
$$

for $\xi \in D_{0}$ and $0<\rho<\delta_{2}$. Now take

$$
\delta=\frac{\delta_{1} \delta_{2}}{\delta_{1}+\delta_{2}}
$$

so that

$$
0<\delta<\delta_{1}, \quad 0<\delta<\delta_{2} .
$$

Set $D_{1}=D_{0} D$. Observe that $\phi\left(D_{1}\right)=\phi\left(D_{0} D\right)=\Phi\left(D_{0}\right)>0$ and that

$$
\alpha \leqq \frac{\Phi\left(\Omega_{\xi}^{p}\right)}{2 \rho}=\frac{\phi\left(D \Omega_{\xi}^{p}\right)}{2 \rho} \leqq \frac{\phi\left(\Omega_{\xi}^{p}\right)}{2 \rho} \leqq 1.01 \alpha
$$

for $\xi \in D_{1}$ and $0<\rho<\delta$. Since the bounded closed set $D_{1}$ is expressible as a finite sum of closed sets each with diameter less than $2^{-1} \delta$ we now can and do select a closed subset $C$ of $D_{1}$ for which 


$$
\operatorname{diam} C<\delta / 2, \quad \phi(C)>0, \quad C \subset D .
$$

Clearly

$$
\begin{array}{ll}
\alpha \leqq \frac{\phi\left(\Omega_{\xi}^{\beta}\right)}{2 \rho} \leqq 1.01 \alpha & \text { for } \xi \in D \text { and } 0<\rho<\delta, \\
\alpha \leqq \frac{\phi\left(D \Omega_{\xi}^{\rho}\right)}{2 \rho} \leqq 1.01 \alpha & \text { for } \xi \in C \text { and } 0<\rho<\delta .
\end{array}
$$

Having effected this uniformization we put

$$
\mu=.06 \alpha
$$

and suppose $p \in C, q \in C$. We shall complete the proof by showing that

$$
\phi(p * q) \geqq \mu|q-p| \text {. }
$$

To this end let $d=|q-p|$, note that (4) is trivially true if $d=0$, assume henceforth that $d>0$, let $P=(0,0), Q=(1,0)$, and $T$ be the function on $E_{2}$ (that is the finite complex plane) such that

$$
T(x)=(q-p) x+p
$$

for $x \in E_{2}$.

Clearly $T$ is a continuous, univalent function whose domain and whose range is $E_{2}$, indeed for $x \in E_{2}$ and $y \in E_{2}$ we have

$$
|T(y)-T(x)|=d|y-x| \text {. }
$$

Introduce the abbreviations

$$
x^{\prime}=T(x), \quad \bar{s}=\sum_{x \in \cdot}\left\{x^{\prime}\right\}
$$

check that

$$
\overline{s S}=\bar{s} \bar{S}, \quad \overline{s \Omega_{x}^{r}}=\bar{s} \Omega_{x^{\prime}}^{r d}
$$

whenever $x \in E_{2}, r>0, s \subset E_{2}, S \subset E_{2}$; define $\psi$ as that function on $\varepsilon_{2}$ such that

$$
\psi(s)=\frac{1}{\alpha d} \phi(\bar{s})
$$

for $s \subset E_{2}$.

Verify that $\psi \in \mathfrak{M}$ and that

$$
\frac{\psi\left(s \Omega_{x}^{r}\right)}{2 r}=\frac{1}{\alpha} \frac{\phi\left(\bar{s} \Omega_{x^{\prime}}^{r d}\right)}{2 r d}
$$

whenever $x \in E_{2}$ and $0<r<\infty$. Determine sets $A$ and $B$ so that

$$
\bar{A}=C, \quad \bar{B}=D .
$$

Since $x \in B$ implies $x^{\prime} \in D$, since $x \in A$ implies $x^{\prime} \in C$, and since $0<r \leqq 2$ 
implies $0<r d \leqq 2 \operatorname{diam} C<\delta$, it follows from (5), (1), and (2) that

$$
\begin{aligned}
& 1 \leqq \frac{\psi\left(\Omega_{x}^{r}\right)}{2 r} \leqq 1.01 \quad \text { for } x \in B \text { and } 0<r \leqq 2 \text {, } \\
& 1 \leqq \frac{\downarrow\left(B \Omega_{x}^{r}\right)}{2 r} \leqq 1.01 \text { for } x \in A \text { and } 0<r \leqq 2 \text {. }
\end{aligned}
$$

Recalling that $P=(0,0), Q=(1,0)$ we have

$$
A \subset B, \quad p=P^{\prime}, \quad q=Q^{\prime}, \quad P \in A, \quad Q \in A,
$$

so that Theorem 8.1 implies

$$
\psi(P * Q)>.06
$$

A simple check shows

$$
\overline{P * Q}=p * q
$$

from which we deduce

$$
\frac{1}{\alpha d} \phi(p * q)=\frac{1}{\alpha d} \phi(\overline{P * Q})=\psi(P * Q)>.06 .
$$

With the help of (3) we thus conclude that

$$
\phi(p * q)>.06 \alpha d=.06 \alpha|q-p|=\mu|q-p|
$$

and that (4) is true.

8.3. THEOREM. If $\phi \in \mathfrak{M}, 3 \mathfrak{D}_{\phi}^{\Delta}(x)<4 \mathfrak{D}_{\phi}^{\nabla}(x)$ for $\phi$ almost all $x$, then there is a closed set $C$ with $\phi(C)>0$ and $a$ number $\mu>0$ such that

$$
\phi(p * q) \geqq \mu|q-p|
$$

for $p \in C$ and $q \in C$.

Proof. By Lemma 5.10 there are positive numbers $\alpha, \delta$, $\eta$ with $1<\eta<4 / 3$ and a bounded closed set $C^{\prime}$ with $\phi\left(C^{\prime}\right)>0$ such that the relations $x \in C^{\prime}$, $\Gamma$ is a circle with $x \in \Gamma, 0<2 r<\delta$, diam $\Gamma<\delta$ together imply the relations

$$
\alpha \leqq \frac{\phi\left(\Omega_{x}^{*}\right)}{2 r}, \quad \frac{\phi(\Gamma)}{\operatorname{diam} \Gamma} \leqq \alpha \eta .
$$

Since $C^{\prime}$ is expressible as a finite sum of closed sets each with diameter less than $3^{-1} \delta$, we choose a closed subset $C$ of $C^{\prime}$ with $\phi(C)>0$ and diameter less than $3^{-1} \delta$.

Putting $\mu=\alpha(4-3 \eta)$, observing the positivity of $\mu$, and supposing $p \in C$ and $q \in C$ we propose to complete the proof by showing 


$$
\phi(p * q) \geqq \mu|q-p|
$$

To this end let $d=|q-p|$, note that (2) is trivially true if $d=0$, assume henceforth that $d>0$, and define

$$
p^{\prime}=\frac{p+q}{2}, \quad \Gamma_{1}=\Omega_{p}^{d}, \quad \Gamma_{2}=\Omega_{q}^{d}, \quad \Gamma_{3}=\Omega_{p^{\prime}}^{1.5 d} .
$$

Since $x \in \Gamma_{1}$ implies $\left|x-p^{\prime}\right| \leqq|x-p|+\left|p-p^{\prime}\right|<d+.5 d$ and since $x \in \Gamma_{2}$ implies $\left|x-p^{\prime}\right| \leqq|x-q|+\left|q-p^{\prime}\right|<d+.5 d$ we see that $\Gamma_{1}+\Gamma_{2} \subset \Gamma_{3}$. Accordingly

$$
\phi\left(\Gamma_{3}\right) \geqq \phi\left(\Gamma_{1}\right)+\phi\left(\Gamma_{2}\right)-\phi\left(\Gamma_{1} \Gamma_{2}\right) .
$$

From this, (1), and the fact that $2 d \leqq 3 d \leqq 3$ diam $C<\delta$ it follows that

$$
3 d \eta \alpha \geqq 4 d \alpha-\phi\left(\Gamma_{1} \Gamma_{2}\right)=4 d \alpha-\phi(p * q)
$$

and hence that

$$
\phi(p * q) \geqq \alpha(4-3 \eta) d=\mu|q-p| .
$$

With the verification of (2) the proof is complete.

9. Density ratios and rectifiability.

9.1. Theorem. If

(i) $\phi \in \mathfrak{M}$ and $\mu>0$,

(ii) $0<\mathfrak{D}_{\phi}^{\Delta}(x)<\infty$ for $\phi$ almost all $x$,

(iii) $C$ is a bounded closed set with $\phi(C)>0$,

(iv) $\phi(p * q) \geqq \mu|q-p|$ for $p \in C$ and $q \in C$,

then there is a rectifiable set of positive $\phi$ measure.

Proof. We determine: according to Lemma 5.4, a number $\alpha>0$ and a closed set $A$ such that

$$
\phi(\tilde{A})<\phi(C) / 4
$$

and

$$
\mathfrak{D}_{\phi}^{\Delta}(x)>\alpha
$$

for $x \in A$;

and next, according to Lemma 5.7, a number $\lambda>1$ and a closed set $B$ such that

$$
\phi(\tilde{B})<\phi(C) / 4
$$

and

$$
\phi\left(\Omega_{x}^{r}\right) \leqq 2 r \lambda
$$

for $x \in B$ and $0<r<\lambda^{-1}$. Let $C_{1}=A B C$, note that $C_{1}$ is closed and that

$$
\phi\left(C_{1}\right)>\phi(C) / 2>0 \text {, }
$$




$$
\begin{aligned}
& \mathfrak{D}_{\phi}^{\Delta}(x)>\alpha>0 \\
& \phi\left(\Omega_{x}^{r}\right) \leqq 2 r \lambda
\end{aligned}
$$$$
\text { for } x \in C_{1} \text {, }
$$$$
\text { for } x \in C_{1} \text { and } 0<r<1 / \lambda \text {. }
$$

Let $\epsilon$ be a number such that both

$$
0<\epsilon<\frac{\alpha \mu}{2+240 \lambda}
$$

and

$$
0<\epsilon<\alpha / 2 \text {. }
$$

We use 5.12 and 5.26 to see that $\mathfrak{D}_{\phi}^{\Delta}\left(\bar{C}_{1}, x\right)=0$ for $\phi$ almost all $x$ in $C_{1}$ and now ascertain $x_{0} \in C_{1}$ and a number $\delta$ such that

$$
0<\delta<1 / \lambda
$$

and

$$
\frac{\phi\left(C_{1} \Omega_{x_{0}}^{r}\right)}{2 r}<\epsilon
$$$$
\text { for } 0<r<\delta \text {. }
$$

Next, relying on (1), we let $r_{0}$ be a number such that

$$
\begin{aligned}
& 0<r_{0}<\delta / 12, \\
& \phi\left(\Omega_{x_{0}}^{r_{0}}\right)>\alpha r_{0} .
\end{aligned}
$$

and note, using (4) and recalling 2.9,

$$
\phi\left(C_{1} \mathcal{C}_{x_{0}}^{r_{0}}\right) \geqq \phi\left(C_{1} \Omega_{x_{0}}^{r_{0}}\right)=\phi\left(\Omega_{x_{0}}^{r_{0}}\right)-\phi\left(\tilde{C}_{1} \Omega_{x_{0}}^{r_{0}}\right)>\alpha r_{0}-2 r_{0 \in}>0 .
$$

Let

$$
C_{2}=C_{1} \mathfrak{G}_{x_{0}}^{n_{0}} \text { where } r_{1}=\inf \underset{t}{E}\left[\mathfrak{G}_{x_{0}}^{t} \supset C_{1} \mathfrak{C}_{x_{0}}^{r_{0}}\right]
$$

and check that

$$
C_{1} \mathfrak{E}_{x_{0}}^{r_{1}}=C_{1} \mathfrak{E}_{x}^{r_{0}}
$$

and

$$
C_{1} \text { bndr } \mathfrak{夭}_{x_{0}}^{n_{1}} \neq 0 \text {. }
$$

It is clear that $r_{1} \leqq r_{0}$, but we observe furthermore that

$$
0<r_{1} \leqq r_{0} ;
$$

for if it were possible that $r_{1}=0$, it would follow that $C_{2}=\left\{x_{0}\right\}$ and that

$$
\phi\left(\left\{x_{0}\right\}\right)=\phi\left(C_{2}\right)=\phi\left(C_{1}\left(\mathfrak{E}_{x_{0}}^{r_{1}}\right)=\phi\left(C_{1}\left(\mathfrak{S}_{x_{0}}^{r_{0}}\right)>0 \text { and } \mathfrak{D}_{\phi}^{\Delta}\left(x_{0}\right)=\infty\right. \text {, }\right.
$$


contrary to (2). Therefore

$$
\phi\left(C_{2}\right)>r_{0}(\alpha-2 \epsilon) \geqq r_{1}(\alpha-2 \epsilon) .
$$

We define

$$
\gamma=\operatorname{bndr} \Omega_{x_{0}}^{r_{1}}
$$

Since bndr $\Omega_{x_{0}}^{r_{1}}=$ bndr $C_{x_{0}}^{\gamma_{1}}$ we have from (8)

$$
0 \neq \gamma C_{1} \mathfrak{S}_{x_{0}}^{r_{0}} \subset \gamma C_{2} \text {. }
$$

Let $\mathfrak{F}$ be the family of circles such that $\Gamma \in \mathfrak{F}$ if and only if there is an $x$ and an $r$ such that $\Gamma=\Omega_{x}^{r}$ where

$$
x \in C_{2}, 0<r<2 r_{1} \text {, and } \phi\left(\tilde{C}_{1} \Omega_{x}^{r}\right) \geqq \mu r \text {. }
$$

Now, using the covering Theorem 5.11, we let $\$$ be a countable disjointed subfamily of $\mathfrak{F}$ such that

$$
\sum_{s \in \mathbb{S}} \hat{s} \supset \sigma(\mathfrak{F})
$$

Clearly for each $d>0$

$$
\text { (s) } E[(\operatorname{diam} s)>d] \text { is finite. }
$$

Therefore considering the family $\sum_{s \in \Theta}\{s\}$ in relation to Theorem 4.1, we select a subfamily $\mathfrak{S}$ of $\mathbb{S}$ such that the family

$$
\sum_{\bullet \in \Phi}\{\hat{s}\}
$$

is semi-disjointed and

$$
\sum_{. \in \Phi} s=\sum_{\bullet \in \Theta} \hat{s}
$$

We now let

$$
C_{3}=C_{2}+\gamma+\sum_{: \in \mathfrak{G}}(\operatorname{clsr} \hat{s})
$$

and

$$
D=\left(C_{3}-\sum_{\cdot \in \mathfrak{\Phi}} s\right)+\sum_{: \in \mathfrak{S}}(\operatorname{bndr} \hat{s}),
$$

and divide the remainder of the proof into six parts.

PART I. $C_{3}$ is closed.

Proof (by contradiction). Suppose $p_{0} \in \widetilde{C}_{3}\left(\operatorname{clsr} C_{3}\right)$. Since $\left(C_{2}+\gamma\right)$ is closed and clsr $s$ is closed for each $s \in \mathfrak{W}$, we select distinct members $s_{1}, s_{2}, s_{3}, \cdots$ of $\$$ with 


$$
\lim _{n \rightarrow \infty} \operatorname{dist}\left[\left\{p_{0}\right\},\left(\operatorname{clsr} \hat{s}_{n}\right)\right]=0 .
$$

From (12) we infer

$$
\lim _{n \rightarrow \infty}\left(\operatorname{diam} \hat{s}_{n}\right)=0 .
$$

Thus, since the center of each of these circles is an element of $C_{2}$, it follows that $p_{0}$ is a cluster point of the closed set $C_{2}$, and $p_{0} \in C_{2} \subset C_{3}$.

PART II. $C_{3}$ is connected.

Proof (by contradiction). Suppose $C^{\prime}$ and $C^{\prime \prime}$ are disjoint nonvacuous closed sets with $C_{3}=C^{\prime}+C^{\prime \prime}, \gamma \subset C^{\prime}$. Evidently

$$
(\operatorname{clsr} s) \subset C^{\prime} \text { or }(\operatorname{clsr} s) \subset C^{\prime \prime} \quad \text { for } s \in \mathfrak{W} \text {. }
$$

To show that $C^{\prime \prime} \subset \mathbb{S}_{x_{0}}^{r_{1}}$ we assume the contrary: there is a circle $s \in \mathfrak{G}$ with (clrs $s) \subset C^{\prime \prime}$, (clsr $\left.s\right) \tilde{\mathcal{S}}_{x_{0}}^{r_{1}} \neq 0$. Since the center of $s$ is in $C_{2} \subset \mathfrak{G}_{x_{0}}^{r_{1}}$ we have $0 \neq(\operatorname{clsr} s) \gamma \subset C^{\prime \prime} C^{\prime}=0$.

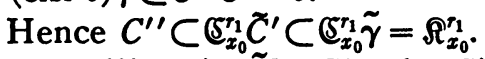

Let $C^{\prime \prime \prime}=C^{\prime}+\tilde{\Omega}_{x_{0}}^{r_{1}}$. Clearly $C^{\prime \prime \prime}$ is closed with $C^{\prime \prime \prime} C^{\prime \prime}=0$. It is evident that $0 \neq C_{1} C^{\prime \prime}, 0 \neq C_{1} C^{\prime} \subset C_{1} C^{\prime \prime \prime}$. Choose points $p \in C_{1} C^{\prime \prime}$ and $q \in C_{1} C^{\prime \prime \prime}$ such that

$$
|p-q|=\operatorname{dist}\left(C_{1} C^{\prime \prime}, C_{1} C^{\prime \prime \prime}\right)>0 \text {. }
$$

Since $\gamma C_{1} \neq 0$ by $(10)$ and since $p \in \Omega_{x_{0}}^{r_{1}}$ we have $|p-q|<2 r_{1}$. Obviously $(p * q) \subset \tilde{C}_{1}, p \in C_{2}$ and (iv) implies

$$
\phi\left(\Omega_{p}^{|p-q|} \tilde{C}_{1}\right) \geqq \phi(p * q) \geqq \mu|p-q| .
$$

Hence $\Omega_{p}^{|p-q|} \in \mathfrak{F}, \mathfrak{C}_{p}^{|p-q|}$ is contained in $C_{3}$, is a continuum, and is split into two disjoint closed sets $C^{\prime \prime} \mathfrak{G}_{p}^{|p-q|}$ and $C^{\prime \prime \prime} \mathfrak{\complement}_{p}^{|p-q|}$ with $p$ in the first and $q$ in the second.

PART III. $D$ is a continuum.

Proof. By Parts I and II the set $C_{3}$ is a continuum. From Lemma 4.7 it follows that $D$ is a continuum.

PART IV. $L(D)<\infty$.

Proof. Evidently

$$
D \subset C_{2}+\gamma+\sum_{\cdot \in \mathfrak{W}}(\text { bndr } s) \text {. }
$$

Noting that $\mathfrak{E}$ is disjointed and $\phi \in \mathfrak{M}$ we use (11) to obtain

$$
\sum_{\mathscr{S} \in \mathfrak{S}} \operatorname{diam} s \leqq \frac{2}{\mu} \sum_{\mathscr{s} \in \mathfrak{S}} \phi\left(\tilde{C}_{1} s\right) \leqq \frac{2}{\mu} \sum_{\mathscr{s} \in \mathfrak{S}} \phi(s) \leqq \frac{2}{\mu} \phi[\sigma(\mathfrak{S})]<\infty,
$$

$$
L\left[\sum_{\mathscr{s} \in \mathfrak{S}}(\operatorname{bndr} s)\right] \leqq 5 \pi \sum_{\mathscr{s} \in \mathfrak{W}}(\operatorname{diam} s)<\infty \text {. }
$$


Remembering (1) and Lemma 5.20 we have

$$
L\left(C_{2}\right)=L\left(C_{1} \mathfrak{S}_{x_{0}}^{r_{1}}\right) \leqq(5 / \alpha) \phi\left(C_{1} \mathfrak{\complement}_{x_{0}}^{r_{1}}\right)<\infty .
$$

Combining (13), (14), (15) and the relation $L(\gamma)=2 \pi r_{1}<\infty$ we reach the conclusion $L(D)<\infty$.

PART V. $D$ is rectifiable.

Proof. $D$ is a continuum of finite Carathéodory linear measure. Hence Theorem 4.6 implies that $D$ is rectifiable.

PART VI. $\phi(D)>0$.

Proof. Since $\sum_{s \in \mathfrak{\Phi}^{\xi}}$ is a Borel subset of $C_{3}$ and $D \supset C_{3}-\sum_{s \in \mathfrak{S}^{\xi}}$ we have

$$
\phi(D) \geqq \phi\left(C_{3}-\sum_{. \in \mathfrak{S}} s\right)=\phi\left(C_{3}\right)-\phi\left(\sum_{. \in \mathfrak{W}} s\right) .
$$

Since $C_{3} \supset C_{2}$ we have, using (9),

$$
\phi\left(C_{8}\right) \geqq \phi\left(C_{2}\right) \geqq r_{1}(\alpha-2 \epsilon) .
$$

Next, since $\mathfrak{S C F}$, we use (11), (7), (5), (2) to obtain the relation

$$
\begin{aligned}
& \phi\left(\sum_{\mathfrak{s} \in \mathfrak{\Phi}} s\right) \leqq \sum_{\mathfrak{s} \in \mathfrak{W}} \phi(\hat{s}) \leqq \lambda \sum_{\mathfrak{s} \in \mathfrak{\Phi}} \operatorname{diam} s=5 \lambda \sum_{\mathscr{s} \in \mathfrak{\Phi}} \operatorname{diam} s \\
& \leqq \frac{10 \lambda}{\mu} \sum_{\bullet \in \mathfrak{\Phi}} \phi\left(\tilde{C}_{1} s\right) \text {. }
\end{aligned}
$$

From the facts that $\mathfrak{W}$ is disjointed, $\sigma(\mathfrak{W}) \subset \Omega_{x_{0}}^{12 r_{1}}$ and from (6) we have that

$$
\sum_{: \in \mathfrak{S}} \phi\left(\tilde{C}_{1} s\right)=\phi\left[\tilde{C}_{1} \sigma(\mathfrak{W})\right] \leqq \phi\left(\tilde{C}_{1} \Re_{x_{0}}^{12 r_{1}}\right)<24 r_{1} \epsilon
$$

and thus, referring back to (16), that

$$
\phi\left(\sum_{. \in \mathfrak{S}} s\right) \leqq \frac{240 \lambda r_{1} \epsilon}{\mu} .
$$

Finally, remembering the bounds for $\epsilon$ given in (3), we have

$$
\phi(D) \geqq r_{1}(\alpha-2 \epsilon)-\frac{240 \lambda r_{1} \epsilon}{\mu}=r_{1}\left[\alpha-\epsilon\left(\frac{(2+240 \lambda)}{\mu}\right)\right]>0 .
$$

We now state three theorems and prove the third; the first two follow from the third.

9.2. THEOREM. If $\phi \in \mathfrak{M}$ and $\mathfrak{D}_{\phi}^{\Delta}(x)<1: 01 \mathfrak{D}_{\phi}^{\nabla}(x)$ for almost all $x$, then $E_{2}$ is $\phi$ rectifiable.

9.3. THEOREM. If $\phi \in \mathfrak{M}$ and $3 \mathfrak{D}_{\phi}^{\Delta}(x)<4 \mathfrak{D}_{\phi}^{\nabla}(x)$ for almost all $x$, then $E_{2}$ is $\phi$ rectifiable. 
9.4. ThEOREM. If $\phi \in \mathfrak{M}$,

$$
A_{1}=\underset{x}{E}\left[\mathfrak{D}_{\phi}^{\Delta}(x)<1.01 \mathfrak{D}_{\phi}^{\nabla}(x)\right], \quad A_{2}=\underset{x}{E}\left[3 \mathfrak{D}_{\phi}^{\Delta}(x)<4 \mathfrak{D}_{\phi}^{\nabla}(x)\right]
$$

and $\phi\left(\tilde{A}_{1}\right) \phi\left(\tilde{A}_{2}\right)=0$, then $E_{2}$ is $\phi$ rectifiable.

Proof. Let $\nu=1$ or 2 according as $\phi\left(\tilde{A}_{1}\right)$ is or is not zero and note that $\phi\left(\tilde{A}_{\nu}\right)=0$.

Letting $B$ be any Borel set with positive $\phi$ measure, we introduce the intersecting measure $\Phi \in \mathfrak{M}$ for which

$$
\Phi(s)=\phi(B s) \quad \text { for } s \subset E_{2},
$$

and note, with the aid of 5.14, that for $L$ almost all $x \in B$

$$
\mathfrak{D}_{\phi}^{\nabla}(x)=\mathfrak{D}_{\phi}^{\nabla}(B, x)=\mathfrak{D}_{\Phi}^{\nabla}(x), \quad \mathfrak{D}_{\phi}^{\Delta}(x)=\mathfrak{D}_{\Phi}^{\vec{\Delta}}(x), \quad \mathfrak{D}_{\phi}^{\Delta}(x)=\mathfrak{D}_{\Phi}^{\Delta}(x) .
$$

Thus defining

$$
D_{1}=\underset{x}{E}\left[\mathfrak{D}_{\Phi}^{\Delta}(x)<1: 01 \mathfrak{D}_{\Phi}^{\nabla}(x)\right], \quad D_{2}=\underset{x}{E}\left[3 \mathfrak{D}_{\Phi}^{\Delta}(x)<4 \mathfrak{D}_{\Phi}^{\nabla}(x)\right],
$$

noting that $\mathfrak{D}_{\phi}^{\Delta}(x)<\infty$ for $\phi$ almost all $x$ and using 5.26 we obtain

$$
0=L\left(B A_{v} \tilde{D}_{v}\right)=\phi\left(B A_{v} \tilde{D}_{v}\right)=\phi\left(B A_{\nu} \widetilde{D}_{v}\right)+\phi\left(B \tilde{A}_{\nu} \tilde{D}_{v}\right)=\phi\left(B \tilde{D}_{v}\right)=\Phi\left(\widetilde{D}_{v}\right) .
$$

Relying upon 8.2 or 8.3 according as $\nu=1$ or $\nu=2$, we select a number $\mu>0$ and a bounded closed set $C$ such that $\Phi(C)>0$ and

$$
\Phi(p * q) \geqq \mu|q-p|
$$

for $p \in C$ and $q \in C$. Clearly

$$
0<\mathfrak{D} \stackrel{\Delta}{\Phi}(x)<\infty
$$

for $\Phi$ almost all $x$ so that we may now invoke 9.1 to ascertain a rectifiable set $\beta$ of positive $\Phi$ measure. From this it follows at once that $B \beta$ is a rectifiable subset of $B$ with

$$
\phi(B \beta)=\Phi(\beta)>0 .
$$

Recourse to Lemma 7.14 completes the proof.

By using intersecting measure we obtain the following two theorems from 9.2 and 9.3 respectively.

9.5. THEOREM. If $\phi \in \mathfrak{U}, \phi(A)<\infty, \mathfrak{D}_{\phi}^{\Delta}(A, x)<1.01 \mathfrak{D}_{\phi}^{\nabla}(A, x)$ for $\phi$ almost all $x$ in $A$, then $A$ is $\phi$ rectifiable.

9.6. THEOREM. If $\phi \in \mathfrak{U}, \phi(A)<\infty, 3 \mathfrak{D}_{\phi}^{\Delta}(A, x)<4 \mathfrak{D}_{\phi}^{\nabla}(A, x)$ for $\phi$ almost all $x$ in $A$, then $A$ is $\phi$ rectifiable. 


\section{Rectifiability and density ratios.}

10.1. ThEOREM. If $\phi \in \mathfrak{M}, \mathfrak{D}_{\phi}^{\nabla}(x)<\infty$ for $\phi$ almost all $x$, and $A$ is strictly rectifiable, then

$$
0<\mathfrak{D}_{\phi}^{\nabla}(x)=\mathfrak{D}_{\phi}^{\Delta}(x)<\infty
$$

for $\phi$ almost all $x$ in $A$.

Proof. Letting

$$
\begin{aligned}
C^{\prime} & =A-\underset{x}{E}\left[\mathfrak{D}_{L}^{\nabla}(A, x)=\mathfrak{D}_{L}^{\Delta}(A, x)=1,\right. \\
C^{\prime \prime} & =A \underset{x}{E}\left[\mathfrak{D}_{\phi}^{\Delta}(x)=\infty\right], \\
C^{\prime \prime \prime} & =A \underset{x}{E}\left[\mathfrak{D}_{\phi}^{\nabla}(x)=0\right],
\end{aligned}
$$

we see in the light of 5.29 that any subset of $A$ which has $L$ measure zero has $\phi$ measure zero, and use $6.12,5.19$ and $5.13,5.28$ to obtain

$$
0=L\left(C^{\prime}\right)=\phi\left(C^{\prime}\right), \quad 0=L\left(C^{\prime \prime}\right)=\phi\left(C^{\prime \prime}\right), \quad 0=\phi\left(C^{\prime \prime \prime}\right) .
$$

Thus

$$
\phi\left(A-A^{\prime}\right)=0
$$

where

$$
A^{\prime}=A-\left(C^{\prime}+C^{\prime \prime}+C^{\prime \prime \prime}\right) \text {. }
$$

Let $\mathcal{L}$ be the function on $\varepsilon_{2}$ such that $\mathcal{L}(s)=L(A s)$ for $s \subset E_{2}$, note $\mathcal{L} \in \mathfrak{M}$ and that

$$
0<\mathfrak{D}_{\phi}^{\nabla}(x) \leqq \mathfrak{D}_{\phi}^{\Delta}(x)<\infty, \quad \mathfrak{D}_{\mathcal{L}}^{\nabla}(x)=\mathfrak{D}_{\hat{\mathcal{L}}}(x)=1
$$

for each $x \in A^{\prime}$.

Let $V$ be the function on $A^{\prime}$ such that $x \in A^{\prime}$ implies $V(x)$ is the family of circles of the form $\Omega_{x}^{r}$ where

$$
\phi\left(\Omega_{x}^{b r}\right)<6 \frac{\mathfrak{D}_{\phi}^{\Delta}(x)}{\mathfrak{D}_{\phi}(x)} \phi\left(\Omega_{x}^{r}\right) .
$$

Let $F$ be the function on $A^{\prime}$ such that $x \in A^{\prime}$ implies $F(x)$ is the family of those circles $\Gamma$ for which $x \in \Gamma$ and

$$
\phi(\widehat{\Gamma})<6 \frac{\mathfrak{D}_{\phi}^{\Delta}(x)}{\mathfrak{D}_{\phi}^{\nabla}(x)} \phi(\Gamma) .
$$

Note $V(x) \subset F(x)$ whenever $x \in A^{\prime}$. 
Notation 2.9 of [5] is used in the next three of the four parts into which the remainder of the proof is divided.

PART I. If $x \in A^{\prime}$, then

$$
\varliminf_{V(x) \ni \Gamma \rightarrow x} \frac{\phi(\Gamma)}{\operatorname{liam} \Gamma}=\mathfrak{D}_{\phi}^{\nabla}(x) .
$$

Proof. Let $r_{1}, r_{2}, r_{8}, \cdots$ be positive numbers such that: $r_{n} \rightarrow 0$ as $n \rightarrow \infty$; and

$$
\mathfrak{D}_{\phi}^{\nabla}(x)=\lim _{n \rightarrow \infty} \phi\left(\Omega_{x}^{r_{n}}\right) \text {. }
$$

We have

$$
\varlimsup_{n \rightarrow \infty} \frac{\phi\left(\Omega_{x}^{5 r_{n}}\right)}{\phi\left(\Omega_{x}^{r_{n}}\right)}=5 \varlimsup_{n \rightarrow \infty} \frac{\phi\left(\Omega_{x}^{5 r_{n}}\right)}{10 r_{n}} \cdot \frac{2 r_{n}}{\phi\left(\Omega_{x}^{r_{n}}\right)} \leqq 5 \frac{\mathfrak{D}_{\phi}^{\Delta}(x)}{\mathfrak{D}_{\phi}^{\nabla}(x)}<6 \frac{\mathfrak{D}_{\phi}^{\Delta}(x)}{\mathfrak{D}_{\phi}^{\nabla}(x)} .
$$

Accordingly for $n$ sufficiently large

$$
\phi\left(\Omega_{x}^{b \tau_{n}}\right)<6 \frac{\mathfrak{D}_{\phi}^{\Delta}(x)}{\mathfrak{D}_{\phi}^{\nabla}(x)} \phi\left(\Omega_{x}^{r_{n}}\right)
$$

and $\Omega_{x}^{r_{n}} \in V(x)$. From this and (3) we conclude

$$
\mathfrak{D}_{\phi}^{\nabla}(x) \leqq \underset{V(x) \ni \Gamma \rightarrow x}{\operatorname{limal}} \frac{\phi(\Gamma)}{\operatorname{diam} \Gamma} \leqq \mathfrak{D}_{\phi}^{\nabla}(x) .
$$

Part II. If $x \in A^{\prime}$, then

$$
\mathfrak{D}_{\phi}^{\Delta}(x)=\varlimsup_{\boldsymbol{\Gamma}(x) \ni \Gamma \rightarrow x} \frac{\phi(\Gamma)}{\operatorname{diam} \Gamma} .
$$

Proof. Let $\Gamma_{1}, \Gamma_{2}, \Gamma_{3}, \cdots$ be circles, each of which has $x$ in it, with

$$
\operatorname{diam} \Gamma_{n} \rightarrow 0 \text { and } \frac{\phi\left(\Gamma_{n}\right)}{\operatorname{diam} \Gamma_{n}} \rightarrow \mathfrak{D}_{\phi}^{\wedge}(x)
$$

as $n \rightarrow \infty$. For each positive integer $n$ we set $d_{n}=\operatorname{diam} \Gamma_{n}$ and obtain

$$
\varlimsup_{n \rightarrow \infty} \frac{\phi\left(\widehat{\Gamma}_{n}\right)}{\phi\left(\Gamma_{n}\right)}=5 \varlimsup_{n \rightarrow \infty} \frac{\phi\left(\widehat{\Gamma}_{n}\right)}{5 d_{n}} \cdot \frac{d_{n}}{\phi\left(\Gamma_{n}\right)} \leqq 5 \frac{\mathfrak{D}_{\phi}^{\Delta}(x)}{\mathfrak{D}_{\phi}(x)}<6 \leqq 6 \frac{\mathfrak{D}_{\phi}^{\Delta}(x)}{\mathfrak{D}_{\phi}(x)} .
$$

Thus for $n$ sufficiently large $\Gamma_{n} \in F(x)$. From this and (4) we conclude

$$
\mathfrak{D}_{\phi}^{\Delta}(x) \leqq \varlimsup_{\Gamma(x) \exists \Gamma \rightarrow x} \frac{\phi(\Gamma)}{\operatorname{diam} \Gamma} \leqq \mathfrak{D}_{\phi}^{\Delta}(x) .
$$

PART III. For $\phi$ almost all $x$ in $A^{\prime}$ we have 


$$
0<\mathfrak{D}_{\phi}^{\nabla}(x)=\mathfrak{D}_{\phi}^{\Delta}(x)<\infty .
$$

Proof. Remembering (2), the definition of $F$ and Parts I and II we see that $F$ is a diametrically regular Borelish blanket. (In [5] see Definitions 6.1, 6.5, 6.6.) Denoting

$$
A^{\prime} \underset{x}{E}\left[0 \leqq \operatorname{limal}_{\Gamma(x) \ni \Gamma \rightarrow x} \frac{\mathcal{L}(\Gamma)}{\phi(\Gamma)}<\infty\right]
$$

by $A^{\prime \prime}$ we use Definition 2.3 and Theorem 11.4 of [5] to gain the information that $\phi\left(A^{\prime}-A^{\prime \prime}\right)=0$.

For $x \in A^{\prime \prime}$ we denote the number

by $h(x)$.

$$
\operatorname{limal}_{\Gamma(x) \ni \Gamma \rightarrow x} \frac{\mathcal{L}(\Gamma)}{\phi(\Gamma)}
$$

Now

$$
\frac{\mathcal{L}(\Gamma)}{\phi(\Gamma)} \cdot \frac{\phi(\Gamma)}{\operatorname{diam} \Gamma}=\frac{\mathcal{L}(\Gamma)}{\operatorname{diam} \Gamma}
$$

whenever $\Gamma \in F(x)$ and $x \in A^{\prime \prime}$. Thus using Part $\mathrm{I}$, the inclusion of $V(x)$ in $F(x)$, (5), the definition of $V$, and (2) we deduce that $x \in A^{\prime \prime}$ implies

$$
\begin{aligned}
h(x) \mathfrak{D}_{\phi}^{\nabla}(x) & =h(x) \underset{V(x) \ni \Gamma \rightarrow x}{\operatorname{limal}} \frac{\phi(\Gamma)}{\operatorname{diam} \Gamma}=\operatorname{limal}_{V(x) \ni \Gamma \rightarrow x} \frac{\mathcal{L}(\Gamma)}{\phi(\Gamma)} \underset{V(x) \ni \Gamma \rightarrow x}{\operatorname{limal}} \frac{\phi(\Gamma)}{\operatorname{diam} \Gamma} \\
& \geqq \underset{V(x) \ni \Gamma \rightarrow x}{\operatorname{limal}} \frac{\mathcal{L}(\Gamma)}{\operatorname{diam} \Gamma} \geqq \mathfrak{D}^{\nabla} \mathcal{L}(x)=1 .
\end{aligned}
$$

On the other hand using Part II, (5), the definition of $F$, and (2) we deduce that $x \in A^{\prime \prime}$ implies

$$
\begin{aligned}
h(x) \mathfrak{D}_{\phi}^{\mathbf{A}}(x) & =h(x) \varlimsup_{\Gamma(x) \ni \Gamma \rightarrow x} \frac{\phi(\Gamma)}{\operatorname{diam} \Gamma}=\operatorname{limal}_{\Gamma(x) \ni \Gamma \rightarrow x} \frac{\mathcal{L}(\Gamma)}{\phi(\Gamma)} \varlimsup_{\Gamma(x) \exists \Gamma \rightarrow x} \frac{\phi(\Gamma)}{\operatorname{diam} \Gamma} \\
& \leqq \varlimsup_{\Gamma(x) \ni \Gamma \rightarrow x} \frac{\mathcal{L}(\Gamma)}{\operatorname{diam} \Gamma} \leqq D_{\mathcal{L}}(x)=1 .
\end{aligned}
$$

Accordingly $x \in A^{\prime \prime}$ implies that

$$
1 \leqq h(x) \mathfrak{D}_{\phi}^{\nabla}(x) \leqq h(x) \mathfrak{D}_{\phi}^{\mathbf{A}}(x) \leqq 1
$$

and hence that

$$
0<\mathfrak{D}_{\phi}^{\nabla}(x)=\mathfrak{D}_{\phi}^{\Delta}(x)<\infty .
$$

Since $\phi\left(A^{\prime}-A^{\prime \prime}\right)=0$ the proof of Part III is complete. 
PART IV. For $\phi$ almost all $x$ in $A$ we have

$$
0<\mathfrak{D}_{\phi}^{\nabla}(x)=\mathfrak{D}_{\phi}^{\Delta}(x)<\infty .
$$

Proof. Use (1) and Part III.

10.2. ThEOREM. If $\phi \in \mathfrak{M}, \mathfrak{D}_{\phi}^{\nabla}(x)<\infty$ for $\phi$ almost all $x$ and $E_{2}^{*}$ is $\phi$ rectifiable, then

$$
0<\mathfrak{D}_{\phi}^{\nabla}(x)=\mathfrak{D}_{\phi}^{\Delta}(x)<\infty
$$

for $\phi$ almost all $x$.

Proof. Let $\epsilon>0$, and choose a strictly rectifiable set $C$ such that $\phi(\tilde{C})<\epsilon$. Putting

$$
B=E_{2}-\underset{x}{E}\left[0<\mathfrak{D}_{\phi}^{\nabla}(x)=\mathfrak{D}_{\phi}^{\Delta}(x)<\infty\right]
$$

we are assured by 10.1 that

$$
\phi(B C)=0 .
$$

Accordingly

$$
\phi(B) \leqq \phi(B C)+\phi(B \tilde{C}) \leqq 0+\phi(\widetilde{C})<\epsilon .
$$

10.3. Theorem. If $\phi \in \mathfrak{U}, \phi(A)<\infty, \mathfrak{D}_{\phi}^{\nabla}(A, x)<\infty$ for $\phi$ almost all $x$ in $A$, and $A$ is $\phi$ rectifiable, then

$$
0<\mathfrak{D}_{\phi}^{\nabla}(A, x)=\mathfrak{D}_{\phi}^{\Delta}(A, x)<\infty
$$

for $\phi$ almost all $x$ in $A$.

Proof. Use intersecting measure and 10.2.

11. Interrelations. A unified and fairly complete picture of our previous results may be obtained from

11.1. Theorem. If $\phi \in \mathfrak{U}, \phi(A)<\infty, \mathfrak{D}_{\phi}^{\nabla}(A, x)<\infty$ for $\phi$ almost all $x$ in $A$, then the following nine propositions are equivalent:

(1) $A$ is $\phi$ rectifiable;

(2) $\mathfrak{D}_{\phi}^{\Delta}(A, x)<1.01 \mathfrak{D}_{\phi}^{\nabla}(A, x)$ for $\phi$ almost all $x$ in $A$;

(3) $3 \mathfrak{D}_{\phi}^{A}(A, x)<4 \mathfrak{D}_{\phi}^{\nabla}(A, x)$ for $\phi$ almost all $x$ in $A$;

(4) $0<\mathfrak{D}_{\phi}^{\nabla}(A, x)=\mathfrak{D}_{\phi}^{\Delta}(A, x)<\infty$ for $\phi$ almost all $x$ in $A$;

(5) $0<\mathfrak{D}_{\phi}^{\nabla}(A, x)=\mathfrak{D}_{\phi}^{A}(A, x)<\infty$ for $\phi$ almost all $x$ in $A$;

(6) $A$ is $\phi$ directional at $\phi$ almost all of its points;

(7) $A$ is $\phi$ restricted at $\phi$ almost all of its points;

(8) Corresponding to each positive number $\epsilon$ there is a set $B C A$ such that $\phi(A \widetilde{B})<\epsilon$ and such that $B$ is directional at each of its points;

(9) Corresponding to each positive number $\epsilon$ there is a set $B C A$ such that $\phi(A \widetilde{B})<\epsilon$ and such that $B$ is restricted at each of its points. 
Proof. Our argument consists of 13 steps each of which is given in the left column of the table below with its corresponding supporting reference or comment juxtaposed in the right column.
(1) implies (6)
6.15
(6) implies (7)
7.27
(7) implies (1)
7.22
(1) implies (8)
6.19
(8) implies (9)
7.28
(9) implies (1)
7.29
(1) implies (5)
10.3
(5) implies (3)
obvious
(3) implies (1)
9.6
(1) implies (5)
10.3
(5) implies (4)
obvious
(4) implies (2)
obvious
(2) implies (1)
9.5

The proof is complete.

In the following three theorems we investigate the connection between $\phi$ and $L$ rectifiability.

11.2. TheOREM. If $\phi \in \mathfrak{U}, \phi(A)<\infty, A$ is $L$ rectifiable, and if $\mathfrak{D}_{\phi}^{\Delta}(A, x)$ $<\infty$ for $\phi$ almost all $x$ in $A$, then $A$ is $\phi$ rectifiable.

Proof. Letting $\Phi \in \mathfrak{M}$ be the function on $\varepsilon_{2}$ such that

$$
\Phi(s)=\phi(A s)
$$

the truth of the theorem is evident from 5.25 and the definitions of $\phi$ and $L$ rectifiability.

11.3. REMARK. It is possible to construct a $\phi$ in $\mathfrak{u}$ and a clósed set $A$ such that

$$
\begin{aligned}
\phi(A) & =1, & L(A) & =0, \\
\mathfrak{D}_{\phi}^{\nabla}(A, x) & =0, & \mathfrak{D}_{\phi}^{\Delta}(A, x) & =\infty
\end{aligned}
$$

for $x \in A$.

The set $A$ is thus obviously $L$ rectifiable but by 11.1 (1) and 11.1 (4) it is not $\phi$ rectifiable.

11.4. Theorem. If $\phi \in \mathfrak{U}, L(A)<\infty, A$ is $\phi$ rectifiable, and $\mathfrak{D}_{\phi}^{\Delta}(A, x)>0$ for $L$ almost all $x$ in $A$, then $A$ is $L$ rectifiable.

Proof. Let $\Phi$ be as in 11.2 , let $\epsilon>0$, choose a positive number $\mu$ so small (see 3.1(i)) that $L(A \widetilde{B})<2^{-1} \epsilon$ where $B$ is the Borel set

$$
\underset{*}{E}\left[\mathfrak{D}_{\Phi}^{\Delta}(x)>\mu\right] \text {. }
$$


Letting $C$ be a closed rectifiable set with $\Phi(\tilde{C})<10^{-1} \mu \epsilon$ we are helped by 5.21 to obtain

$$
\begin{aligned}
L(A \tilde{C}) & =L(A B \tilde{C})+L(A \tilde{B} \tilde{C}) \leqq L(B \tilde{C})+L(A \tilde{B}) \\
& \leqq L(B \tilde{C})+\frac{\epsilon}{2} \leqq \frac{5}{\mu} \Phi(B \tilde{C})+\frac{\epsilon}{2} \leqq \frac{5}{\mu} \Phi(\tilde{C})+\frac{\epsilon}{2}<\epsilon .
\end{aligned}
$$

Thus $A C$ is a rectifiable subset of $A$ with $L(A \tilde{C})<\epsilon$.

The $L$ rectifiability of $A$ is established.

11.5. REMARK. Let $A$ be a set for which $L(A)<\infty$ and which is not $L$ rectifiable; the set $\mathcal{A}$ of 7.30 is such a set. Let $\phi$ be the function on $\varepsilon_{2}$ such that $\phi(s)=0$ for $s \subset E_{2}$. Clearly $A$ is $\phi$ and not $L$ rectifiable.

As an immediate consequence of the two preceding theorems we have

11.6. TheOREM. If $\phi \in \mathfrak{U}, \phi(A)<\infty, L(A)<\infty, \mathfrak{D}_{\phi}^{\Delta}(A, x)>0$ for $L$ almost all $x$ in $A, \mathfrak{D}_{\phi}^{\Delta}(A, x)<\infty$ for $\phi$ almost all $x$ in $A$, then $A$ is $\phi$ rectifiable if and only if $A$ is $L$ rectifiable.

\section{Bibliography}

1. A. S. Besicovitch, On the fundamental geometrical properties of linearly measurable plane sets of points (II). Math. Ann. vol. 115 (1938) pp. 296-329.

2. C. Carathéodory, Über das lineare Mass von Punktmengen-eine Verallgemeinerung des Langenuegriffs, Nachr. Ges. Wiss. Göttingen, 1914, pp. 404-426.

3. H. Hahn, Theorie der reellen Funktionen, vol. 1, Berlin, 1921.

4. C. Kuratowski, Topologie I, Warsaw, 1933. (1944).

5. A. P. Morse, $A$ theory of covering and differentiation, Trans. Amer. Math. Soc. vol. 55

6. F. Roger, Sur quelque applications metriques de la notion de contingent bilateral, C. R. Acad. Sci. Paris vol. 201 (1935) pp. 28-30.

7. S. Saks, Theory of the integral, Warsaw, 1937.

\section{SUPPLEMENTARY Bibliography}

1. T. Estermann, Über Carathéodory und Minkowski Verallgemeinerungen des Längenbegriffs, Abh. Math. Sem. Hamburgischen Univ., 1925, pp. 73-116.

2. J. Favard, Une definition de la longeur et de l'aire, C. R. Acad. Sci. Paris vol. 194 (1932) pp. 344-346.

3. W. Gross, Über des Flächenmass von Punktmengen, Monatshefte für Mathematik und Physik vol. 27 (1918) pp. 145-176.

4. - Über des lineare Mass von Punktmengen, Monatshefte für Mathematik und Physik vol. 28 (1918) pp. 177-193.

5. O. Jansen, Über einige stetige Kurven, über Bogenlängen, linearen Inhalt und Flächeninholt, Inaugural-Dissertation, Königsberg, 1907.

6. R. L. Jeffery, Sets of k-extent in n-dimensional space, Trans. Amer. Math. Soc. vol. 35 (1933) pp. 629-647.

7. H. Minkowski, Über die Begriffe Länge, Oberfläsche und Volumen, Jber. Deutschen Math. Verein. vol. 9 (1900) pp. 115-121. $408-419$. 
9. J. P. Schauder, The theory of surface measure, Fund. Math. vol. 8 (1926) pp. 1-48.

10. H. Steinhaus, Zur Praxis der Rektifikation und zum Langenbegriff, Sächsische Akademie zu Leipzig, Berichte vol. 82 (1930) pp. 120-130.

11. - Sur la portée puratique et théorique de quelques théoremes sur la mesure des ensembles de droites, Comptes rendus du premier congress des pays Slaves, 1929, pp. 348-354.

12. W. H. Young and Grace Chisholm, The theory of sets of points, 1906.

University of California,

Berkeley, Calif.

Cornell University,

ITHACA, N. Y. 